\title{
Les ciències humanes
}

i socials

Perspectives emergents

per als reptes

de les societats multiculturals

Esther Monzó-Nebot (ed.)

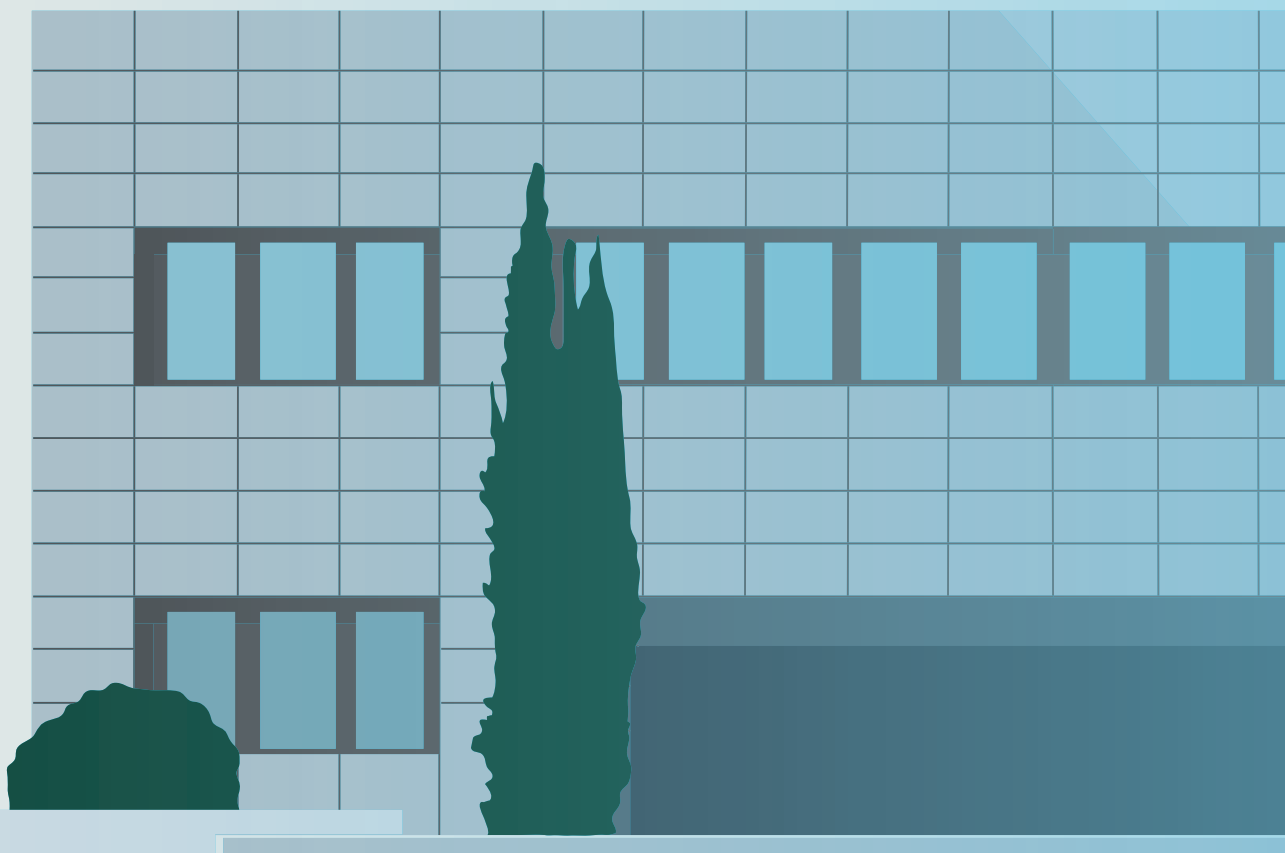


Esther Monzó-Nebot va nàixer a Vila-real el 1976. Llicenciada (1998) i doctora (2002) en Traducció i Interpretació per la Universitat Jaume I, ha estat catedràtica de Sociologia de la Traducció a la Universitat de Graz (Àustria) i és actualment vicedegana de la Facultat de Ciències Humanes i Socials de la Universitat Jaume I. Dedica la seua recerca als aspectes psicosocials de la traducció i la interpretació. 


\title{
Les ciències humanes i socials
}

\author{
Perspectives emergents per als reptes \\ de les societats multiculturals
}




\title{
Direcció de la col-lecció
}

Andreu Casero Ripollés

\section{Comitè científic}

\section{Coordinen}

Esther Monzó-Nebot

Patricia Salazar Campillo

Javier Vellón Lahoz

\author{
Membres \\ Robert Arnau Roselló \\ M. Carmen Campoy Cubillo \\ Hugo Doménech Fabregat \\ Carmen Fernández Nadal \\ Jesús Gil Gómez \\ Jorge Martí Contreras \\ Susana Miquel Segarra \\ Kim Schulte \\ Mónica Velando Casanova
}


Núm. 1

\section{Les ciències humanes i socials}

\section{Perspectives emergents per als reptes de les societats multiculturals}

Esther Monzó-Nebot (ed.)

\section{עل UNAUERSITAT}


Noms: Monzó, Esther, editor literari | Universitat Jaume I. Publicacions, entitat editora

Títol: Les Ciències humanes i socials : perspectives emergents per als reptes de les societats multiculturals / Esther Monzó-Nebot (ed.)

Descripció: Castelló de la Plana : Publicacions de la Universitat Jaume I. Servei de Comunicació i Publicacions, [2020] | Col-lecció: Emergents ; 1 | Textos en valencià, castellà i anglès | Inclou referències bibliogràfiques

Identificadors: ISBN 978-84-17900-71-7 (UJI)

Matèries: Ciències socials | Multiculturalisme | Pluralisme cultural

Classificació: CDU 30 | CDU 316.72 | THEMA JBFB

Publicacions de la Universitat Jaume I és una editorial membre de l'une, cosa que en garanteix la difusió i comercialització de les obres en els àmbits nacional i internacional. www.une.es.

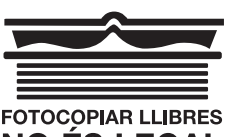

NO ÉS LEGAL
Qualsevol forma de reproducció, distribució, comunicació pública o transformació d’aquesta obra només pot ser realitzada amb l'autorització dels seus titulars, llevat d’excepció prevista per la llei. Dirigiu-vos a CEDro (Centro Español de Derechos Reprográficos, www.cedro.org) si necessiteu fotocopiar o escanejar fragments d'aquesta obra.

(C) Del text: Esther Monzó Nebot (ed.), 2020

Edita: Publicacions de la Universitat Jaume I. Servei de Comunicació i Publicacions Campus del Riu Sec. Edifici Rectorat i Serveis Centrals. 12071 Castelló de la Plana www.tenda.uji.es publicacions@uji.es

ISBN (paper): 978-84-17900-71-7

DOI: http://dx.doi.org/10.6035/Emergents.2020.1

Dipòsit legal: CS 264-2020 


\section{Índex}

La recerca emergent en ciències humanes i socials: fer ciència amb esperit de servei envers les noves societats multiculturals

Esther Monzó-Nebot

To be or not to be: Bridging diverging positions in the case of Charlie Hebdo Irina Charlotte Ranaivosoa

In defence of the Republic: La Vanguardia's plea for multicultural unity in the Spanish Civil War

Samantha N. Edwards

La justicia transicional en perspectiva: los casos de Argentina, Colombia y Sudáfrica

Camilo Eduardo Espinosa Díaz

Considering degrowth from a feminist, transrational peace perspective Marisol C. Bock

The focus on interculturality: A quantitative and qualitative bibliometric study on legal interpreting research Ana Vázquez Fernández

Problemes en la traducció de musicals: estratègies i tècniques per resoldre'ls Joan Alfred Noll Obiol

El adorno en estuco en la Valencia de 1700: de las propuestas autóctonas a la eclosión de la profesión

Gaetano Giannotta 
Estereotipos de género, elección de estudios universitarios y práctica deportiva competitiva en una muestra de deportistas CADU de la Universitat Jaume I Jahel Molina Ulldemolins, M. Carmen Pastor Verchili, María Escrig Mateu, Carlos Hernando Domingo, Sonia Reverter Bañón

Un programa de intervención en psicología positiva para aumentar el bienestar de los docentes

Marisa García Baldán

Notes biogràfiques 163

Bionotes 169

Notas biográficas 175 


\title{
La recerca emergent en ciències humanes i socials: fer ciència amb esperit de servei envers les noves societats multiculturals
}

\author{
Esther Monzó-Nebot \\ monzo@uji.es
}

Resum: El capítol introductori problematitza la visió de ciència en la societat i articula la concepció de les ciències humanes i socials com a camp científic amb una forta idiosincràsia heretada dels objectes destudi que analitza. Des del compromís per la creació sistemàtica i transparent de coneixement, les persones que fan ciència en ciències humanes i socials han demostrat històricament un fort esperit innovador per, sense abandonar el rigor, adaptar les formes de coneixement i desenvolupar perspectives que ens donen informació fefaent i productiva sobre la condició humana i sobre l'ésser humà com a individu en connexió amb d'altres i amb el seu entorn. El capítol fa èmfasi en la capacitat que requereixen les persones dedicades a aquestes ciències per gestionar simultàniament diferents formes de conèixer i per reflexionar constantment sobre la implicació pròpia, com a individus, en la creació de coneixement, des de la selecció dels objectes fins als biaixos possibles en la inferència. La reflexivitat necessària, per a l'individu que investiga i la comunitat que legitima la ciència, és també la millor via per gestionar les societats del futur, multiculturals i amb diversitat creixent.

Paraules clau: ciències humanes i socials, mètode científic, reflexivitat, diversitat, complexitat, societats multiculturals 


\section{La responsabilitat de fer ciència}

Fer ciència comporta una responsabilitat associada. La rendició de comptes no es limita a respondre pels fons que sustenten el sistema científic-que també. La ciència, tot i els discursos que s'entesten a negar-ne els resultats (Antequera 2019), les males pràctiques que porten descrèdit a les institucions i a les publicacions del ram (p. ex., Dahlberg 2018) o els errors d'inferència més o menys provocats per les pressions d'aconseguir publicacions rellevants (Brown i Rohrer 2019; Rippon 2019), continua sent un argument d'autoritat en la nostra societat. S'utilitza per generar la confiança del públic en anuncis de productes farmacèutics, detergents o cosmètics (Medina Cambrón, Sorbías Morales, i Ballano Macías 2007), fins i tot per implicar la superioritat d'unes religions sobre d'altres (Wikipedia 2019). La ciència ofereix encara una pàtina de credibilitat i una resolució definitiva a controvèrsies i dubtes, per la qual la comunitat acadèmica continua responent.

Val a dir que es percep, en el discurs social i fins i tot acadèmic, una distinció entre diferents formes de coneixement. L'apel-latiu de "ciència» no és barat d'aconseguir i les ciències humanes i socials han de bregar dia a dia per fer-se pas en l'imaginari col-lectiu i afiançar que l'estudi sistemàtic de la condició humana i dels éssers humans en la seua interacció amb d'altres i amb l'entorn és fer ciència. No treballem amb bates i provetes, i l'abast de la simbologia que associa les nostres ciències amb la producció de teories que expliquen el món i les validen amb mètodes de rigor contrastat és menys popular. Si les ciències naturals expliquen objectes i fenòmens, les nostres àrees disciplinàries ens ofereixen coneixement sobre conductes i actuacions. La natura diferent dels problemes permet solapaments en diversos àmbits, particularment el mètode científic, però també divergències. El mètode positivista que hem heretat del filòsof Auguste Comte ens diu que treballem amb hipòtesis ex ante i comprovacions objectivants. I amb aquest mètode hem aconseguit part dels avanços assolits en les ciències humanes i socials, però no ens n'ha pogut oferir prou. D'altres paradigmes ho han fet obrint el ventall de metodologies científiques legitimades que, al seu torn, ens han obert la porta a coneixements nous, fent-nos qüestionar creences epistemològiques $i$ provant la validesa i pertinença de superar-les.

Els nous àmbits disciplinaris, els que necessita la societat actual i futura per resoldre els seus problemes més apressants, tenen a l'abast una àmplia gamma d'enfocaments i mètodes per triar, amb la confiança de saber que tots caminen 
per la senda de la creació de coneixement. Les noves ciències beuen dels enfocaments crítics per convèncer la comunitat científica de la pertinença de treballar amb hipòtesis ben argumentades i dels mètodes hermenèutics per posar a prova les inferències, alhora que utilitzen l'empirisme per bastir els fonaments dels resultats i dissenys experimentals per determinar el pes de diferents variables sobre un mateix fenomen complex. Però aquestes són només part de les possibilitats. Les ciències de la conducta, de l'educació, de l'estètica, de la comunicació o del llenguatge, entre d'altres, segueixen posant-se a prova i trobant els punts febles de mètodes, teories i resultats acceptats en altres moments de la història de la ciència. La ciència evoluciona, com ho fa la realitat, i seria illlús i una creença epistemològica poc productiva no acceptar que la comunitat acadèmica és fal-lible i que els resultats temptatius també ens fan avançar. Per molt que ens erigim com el contrari de l'opinió, tota teoria pot veure's superada i, amb això, ajudar-nos a fer visible que la ideologia que impregna les estructures socials travessa disciplines i també és present en els algoritmes que intenten eliminar la participació humana en la generació de resultats científics.

Lésser humà s'entesta a ser present en tot el que fa, diu o pensa. «No veiem mai les coses tal com són, les veiem tal com som» (Nin 1961, 124). Els modes de conèixer han intentat neutralitzar aquesta presència i és encara cert que part del discurs científic segueix alimentant el mite que la neutralitat i l'objectivitat són més que una actitud i un ideal regulatori. Tot i així, periòdicament, la comunitat científica identifica els biaixos del seu coneixement, reconeixent que comprendre'ns i comprendre el món és una meta que cobegem malgrat ser inabastable (Chesterman 1997, 183-184). Darrerament, s'ha fet especialment visible el biaix de gènere, que ha mantingut part de la població en la ignorància de quins símptomes podien alertar les dones d'un atac al cor, per posar només un exemple (Beery 1995). Si neguem els biaixos en lloc d'explorar-los, no fem més que reproduir les mateixes pràctiques, animar els altres a fer el mateix i dificultar que el coneixement avance i la societat millore (vg., per exemple, Régner et al. 2019). Ser conscients dels límits del coneixement i la indagació científica és un pas necessari que les nostres ciències fa temps que van decidir fer, en part, perquè ens hi va obligar la natura dels nostres objectes d'estudi. La complexitat dels fenòmens que estudiem, la diversitat de perspectives que requerim per entendre tots els condicionants i per capturar les dades necessàries, la imbricació d'aquestes perspectives i l'arrelament en contextos locals de les nostres mirades ens porta per camins diferents als que recorren altres models de coneixement. 


\section{La complexitat de mirar la diversitat}

Si alguna cosa caracteritza i distingeix les ciències humanes i socials és la complexitat del seu objecte d'estudi i el reconeixement d'aquesta complexitat en les perspectives d'anàlisi, en els constructes i en els sistemes ontològics que s'han desenvolupat en les nostres disciplines. Els fenòmens pels quals es preocupen les nostres ciències i les relacions que identifiquen i estudien en la realitat que volem conèixer responen a models de dinàmiques de forces més que no als models de causa-efecte que podem identificar amb certa facilitat en les ciències exactes. Per a les ciències humanes i socials, aquestes relacions no serien més que un reduccionisme cartesià de conceptes empeltats en un sistema reticular de relacions i forces, subjectes als canvis que s'esdevenen en tots els punts de la xarxa de la qual fan part.

A més, contrastar les relacions entre fenòmens exigeix una complexitat empírica notable. Identificar tot allò que pot influir en el resultat de les dinàmiques socials és una tasca titànica i poc reductible a condicions de laboratori. No obstant això, els paradigmes de legitimitat i els mètodes d'exploració que considerem científics estan imbuïts de la cultura científica de les ciències exactes, la qual cosa, si s'establira com a model a imitar, podria endarrerir les socials i humanes. Identificar les causes dels efectes més o menys visibles requereix una perspectiva intricada, una suma de representacions i una multiplicitat de mètodes, comporta un redisseny dels processos de refutació i validació, que exigeixen combinacions i triangulacions i que es perllonguen en el temps si es pretén observar els objectes d'estudi en el seu medi i no desproveir-los de la complexitat inherent, la qual cosa falsejaria els resultats i ens apartaria del coneixement desitjat.

Aquesta resistència a les reduccions ens proporciona també, en les ciències humanes i socials, una major diversitat de perspectives. Els objectes que estudiem estan units indissolublement a d'altres en xarxes dinàmiques. En funció de quin fenomen o constructe siga l'inici o l'objecte de les indagacions, la modelació de les dades i de les teories ha de variar. La idea subjacent és que els models d'explicació troben en d'altres complements per explicar la societat i que aplicar-los alternativament o de manera combinada pot limitar les possibilitats de comparació de resultats, però és necessari per enriquir el coneixement sobre com funcionen els grups humans. Aquesta qüestió, però, no és privativa de les nostres àrees de coneixement i només cal veure les defenses aferrissades de les teories universals de la física: si la matèria funciona com a cordes o com a 
partícules crea un cisma ideològic i una polarització de recursos. Una perspectiva habitual en els nostres àmbits seria iniciar una tercera via per cercar si no és que les dues opcions són compatibles. I, just per això, exercir les ciències humanes i socials exigeix transdisciplinarietat, una immersió constant en camps i teories noves, combinables o alternatives, que ens van aclarint el poder explicatiu de les aproximacions i els detalls del que investiguem. Si ens deixem influir per les divisions disciplinàries que ens marca la cultura científica dominant, correm el risc de perdre contacte amb el nostre objecte, que no és cap altre que el que anomenem realitat.

Independentment de la formació inicial amb què ens introduïm en les ciències humanes i socials, la investigadora o l'investigador ha de desenvolupar agilitat intel-lectual per endinsar-se en sistemes ontològics complexos sense diletantisme i gestionar les nocions en el si de sistemes d'interrelacions complexes. Investigar en ciències humanes i socials exigeix poder fer malabars amb conceptes i perspectives, mentalment i lingüísticament. Un factor que adquireix dimensions particulars en les ciències humanes i socials és el llenguatge. En presentar un menor grau de formalització, amb una codificació en llengua comuna, el llenguatge de les nostres àrees revesteix, de fet, un caràcter simbòlic alhora que terminològic. Això té conseqüències en el treball científic, en l'harmonització $i$ en el diàleg entre les perspectives. Termes aparentment comuns com camp o hàbit són altament especialitzats, i representen sistemes amb una complexitat conceptual que comporta fins i tot problemes d'operacionalització, de divisió en nocions que puguem mesurar i contrastar entre estudis diferents. I d'aquesta manera, novament, obtenim complexitat. Creure entendre i estar dialogant pot fer-nos caure en resultats superficials o directament erronis per sinonímia i polisèmia quan intentem trobar la interdisciplinarietat que exigeix una comprensió profunda de la realitat. Aquesta qüestió s’accentua en un món multilingüe on la comunicació en llengües franques ofereix una pàtina d'intercomprensibilitat que de vegades oculta diferències d'arrel en conceptes tan bàsics per a les relacions humanes com la justícia (Peled i Bonotti 2016). Instal-lar-nos en el dubte és, per tant, una competència necessària que abasta tots els nivells.

La dependència dels fenòmens que estudien les ciències humanes i socials de perspectives i evolucions particulars afegeix un nou factor de complexitat. Tant les maneres com ens aproximem als objectes des de l'àmbit acadèmic com l'evolució d'aquests en els seus contextos són producte de la història, de cultures i societats concretes. En certa mesura, aquesta qüestió és rellevant per a totes les disciplines. En els sistemes duals que expliquen el nostre processament de la 
informació (per exemple, Chen i Chaiken 1999; Smith i DeCoster 2000; Strack i Deutsch 2004; Nosek 2007), una primera fase s'activa automàticament sense necessitat de premeditació. Els models disponibles coincideixen a afirmar que aquest primer filtre de la realitat està impregnat de valors culturals, de rutines $\mathrm{i}$ costums de percebre, d'interpretar i d'actuar, els quals hem adquirit en la nostra socialització. I és aquest primer sistema de processament el que anomenem instint i el que molts anomenen sentit comú. Per bé que ens permet ser més eficients en el dia a dia, aquest primer filtre que ens fa creure que entenem el que tenim al davant és, potser, la major amenaça per a la convivència en societats diverses. Si ens quedem amb el que creiem entendre sense qüestionar la percepció, estem assumint les nostres fal.làcies de comunitat, el que assumim que tothom sap, el que tothom pensa que és universal i, més encara, el que entenem com una normalitat prescriptiva. Afortunadament, la ment humana té un segon sistema de processament, que alguns teòrics anomenen sistemàtic, que podem activar i amb el qual les reaccions no són immediates: reflexionem i adoptem punts de vista crítics per reinterpretar el que creiem saber.

Si concebem les ciències humanes i socials com aquelles que expliquen lésser humà, la seua condició i les seues relacions i organitzacions, no podem més que reconèixer el valor central que les creences i valors adquirits en el si d'una cultura i d'una societat tenen en les explicacions. D'una banda hem de contextualitzar els fenòmens, reconèixer que els filtres pels quals avancen els condicionen i, de l'altra, hem de contextualitzar-nos com a narradores. En aquesta tasca són les nostres disciplines les que han posat la solució: reflexivitat. La reflexivitat és l'exercici d'activar el processament sistemàtic, veure'ns i contextualitzar-nos com a investigadores, com a éssers que perceben, interpreten i actuen. Per a tota ciència que requereix, més enllà de la inferència, la interpretació, la reflexivitat esdevé tan essencial com la transparència i la coherència explicativa. Denunciar-nos explícitament com a membres d'un gènere, una classe i un grup social permet que altres puguen mostrar les alternatives des de posicionaments diferents; i reconèixer i manipular els nostres processaments d'informació permet exposar-ne les limitacions i oferir una porta per superar-les. La capacitat de reconèixer, de reflexionar i d'actuar sobre el funcionament de la nostra ment és una condició sine qua non per saber què estem comprenent i des de quins marcs referencials. Per gestionar el coneixement de les ciències humanes i socials, hem de ser reflexives, assumint $a b$ initio la nostra falta de neutralitat i la del coneixement que creiem tenir. Hem de poder gestionar una multitud entrellaçada de sistemes complexos, gestionar la dimensió comunicativa però, molt especialment, la 
dimensió simbòlica del llenguatge, que s'intensifica amb la traducció dels textos font, perquè els conceptes són productes de cultures i de diferències culturals, amb les expressions lingüístiques pròpies. Hem de desenvolupar la capacitat metacognitiva de superar els biaixos de la percepció, la interpretació i l'acció basada en el supòsit de l'existència d'un sentit comú. Més que mai en el món actual, gestionar cultures diferents, també cultures científiques, ens exigeix emmarcarles en els seus sistemes conceptuals i escoltar atentament la diversitat de veus i bagatges culturals de la qual gaudim en l'àmbit internacional de les ciències humanes i socials.

\section{Reptes renovats, noves recerques}

Els beneficis de treballar amb aquests reptes, però, potser no són tan visibles per a les persones que inicien els seus estudis universitaris o les seues carreres investigadores. Les estructures socials que ens envolten són una part responsable d'inculcar-nos les creences que ens mouen. La família, l'escola, però també els mitjans de comunicació tenen molt a dir en el que considerem ciència i el que pensem que podem oferir a través de la recerca (vegeu, per exemple, SchommerAikins, Duell i Barker 2003). Davant la ficcionalització dominant de la vida acadèmica, on els laboratoris, les ulleres de protecció i els acceleradors de partícules s'associen amb les intel-ligències extraordinàries, les eines de desconstrucció crítica, de destrucció creativa i d’emancipació queden a l'ombra, i deixen invisibles possibilitats reals perquè els éssers humans milloren el seu present i el seu futur. Incentivar les noves generacions a progresar en el coneixement de les nostres disciplines és cabdal per fer avançar també la societat, per ensenderar el conjunt de la ciència i assegurar que serveix la humanitat i no la fa esclava.

Aquest volum recull els triomfs de persones i d'institucions que van oferir les bases necessàries perquè joves investigadores i investigadors arribaren al convenciment que fer recerca en ciències humanes i socials és una decisió encertada i una empresa necessària. Les persones que han aportat el seu esforç a aquest volum tenen la convicció que la ciència s'ha d'adaptar als temps i pot oferir les noves societats solucions contundents a problemes concrets en tots els àmbits de l'experiència moderna. Aquesta experiència està caracteritzada per la complexitat i la connectivitat, i tota aproximació científica necessita tenir-les en compte. Les contribucions d'aquest volum acaren aquesta dificultat amb resolució i ens 
ofereixen camins prometedors que ofereixen vistes sobre un dels reptes més importants del nostre temps: els contactes interculturals. Des de mirades al passat, descripcions del present i planificacions del futur, les autores i els autors ens inviten col-lectivament a reconceptualitzar el món en termes humanístics, orgànics, holístics, diversos, multiculturals. Les recerques emergents que recull aquest volum assenyalen qüestions disciplinàries infraestudiades, on les investigadores $\mathrm{i}$ els investigadors revisen les tendències (Vázquez Fernández), s’aventuren creant nous models (Noll Obiol), identificant noves vies (Espinosa Díaz), visibilitzen els problemes de les opcions dominants (Ranaivosoa) i les violències de l'estructura (Molina Ulldemolins et al.), ens recorden com hem perseguit la multiculturalitat (Edwards) des de la fertilització entre sistemes culturals (Giannotta) i ens fan qüestionar-nos les pèrdues i els guanys de permetre'ns construir un món de la mida de la nostra humanitat (Bock i García Baldán).

Les ciències humanes i socials experimenten en aquests moments una rellevància renovada. Són el marc des del qual podem comprendre els canvis que ens envolten. Els canvis tecnològics que ens fan parlar de les societats en xarxa (Castells 1996), les transformacions dels models migratoris que diversifiquen els orígens i els destins alhora que les identitats (Vertovec 2004) i les consciències (Bauman 2000), les noves formes de diversitat lingüística que ens obliguen a modificar paradigmes (Blommaert i Rampton 2011) i a superar els models que ens encoren a categoritzacions improductives (Jain i Wee 2018) ens empenyen, una vegada més, a repensar-nos. La selecció de les propostes que configuren aquest volum, el primer de la col-lecció "Emergents", s'ha guiat per criteris de qualitat i de potencialitat d'impacte. Les persones que són al darrere, les que han fet la recerca, les que les han motivades, les que les han formades i les que els han donat un fòrum per a l'intercanvi i la difusió són responsables de fer-nos avançar decididament cap als models científics del futur. Continuarem resseguint els seus avanços i potenciarem els mecanismes de cooperació, col-laboració i assegurament de la qualitat i de lètica científica preocupant-nos pel producte final, però també pels llocs d’on venim, per on volem arribar i, molt especialment, pel camí que recorrem. 


\section{Bibliografia}

Antequera, José. 2019. «La ciencia se rebela contra Vox». Diario16. https://diario16.com/la-ciencia-se-rebela-contra-vox/.

Bauman, Zygmunt. 2000. Liquid Modernity. Cambridge: Polity.

Beery, Theresa A. 1995. "Gender bias in the diagnosis and treatment of coronary artery disease». Heart \& Lung 24 (6): 427-435. doi: https://doi.org/10.1016/ S0147-9563(95)80020-4.

Blommaert, Jan, i Ben Rampton. 2011. «Language and superdiversity». Diversities $13(2): 1-22$.

Brown, Nicholas J. L., i Julia M. Rohrer. 2019. «Easy as (happiness) pie? A critical evaluation of a popular model of the determinants of well-being». Journal of Happiness Studies. doi: https://doi.org/10.1007/s10902-019-00128-4.

Castells, Manuel. 1996. The Rise of the Network Society. Massachusetts: Blackwell. Chen, Serena, i Shelly Chaiken. 1999. "The heuristic-systematic model in its broader context». A Dual-Process Theories in Social Psychology, editat per Shelly Chaiken i Yaacov Trope, 73-96. Nova York: Guilford.

Chesterman, Andrew. 1997. Memes of Translation. The Spread of Ideas in Translation Theory. Àmsterdam i Filadèlfia: John Benjamins.

Dahlberg, Brett. 2018. "Cornell food researcher's downfall raises larger questions for science». The Salt. https://www.npr.org/sections/ thesalt/2018/09/26/651849441/cornell-food-researchers-downfall-raises-larger-questions-for-science? $\mathrm{t}=1574870136826$.

Jain, Ritu, i Lionel Wee. 2018. «Diversity management and the presumptive universality of categories: The case of the Indians in Singapore». Current Issues in Language Planning 20 (1): 16-32. doi: https://doi.org/10.1080/14664208.2018 .1503386 .

Medina Cambrón, Alfons, Carolina Sorbías Morales i Sonia Ballano Macías. 2007. «La publicidad y sus complejas relaciones con el discurso científico». Questiones Publicitarias: Revista internacional de comunicación y publicidad 1 (12): 77-90.

Nin, Anaïs. 1961. Seduction of the Minotaur. Chicago: The Swallow Press.

Nosek, Brian A. 2007. «Implicit-explicit relations». Current Directions in Psychological Science 16: 65-69. 
Peled, Yael, i Matteo Bonotti. 2016. «Tongue-tied: Rawls, political philosophy and metalinguistic awareness». American Political Science Review 110 (04): 798811. doi: https://doi.org/10.1017/s0003055416000393.

Régner, Isabelle, Catherine Thinus-Blanc, Agnès Netter, Toni Schmader, i Pascal Huguet. 2019. «Committees with implicit biases promote fewer women when they do not believe gender bias exists». Nature Human Behaviour 3: 1171-1179. doi: https://doi.org/10.1038/s41562-019-0686-3.

Rippon, Gina. 2019. The Gendered Brain. Londres: The Bodley Head.

Schommer-Aikins, Marlene, Orpha K. Duell, i Sue Barker. 2003. «Epistemological beliefs across domains using Biglan's classification of academic disciplines». Research in Higher Education 44 (3): 347-366.

Smith, Eliot R., i Jamie DeCoster. 2000. «Dual-process models in social and cognitive psychology: Conceptual integration and links to underlying memory systems». Personality and Social Psychology Review 4: 108-131.

Strack, Fritz, i Roland Deutsch. 2004. "Reflective and impulsive determinants of social behavior». Personality and Social Psychology Review 8: 220-247.

Vertovec, Steven. 2004. «Migrant Transnationalism and Modes of Transformation». International Migration Review 38 (3): 970-1001.

Wikipedia. 2019. «Budismo y ciencia». https://es.wikipedia.org/wiki/Budismo_y_ ciencia. 


\title{
To be or not to be:
}

\section{Bridging diverging positions in the case of Charlie Hebdo $^{1}$}

\author{
Irina Charlotte Ranaivosoa \\ cha.ranaivosoa@live.fr
}

In the aftermath of the 7th January 2015 attacks, when jihadist shot and killed 12 people at the headquarters of the satirical magazine Charlie Hebdo in Paris, the slogan Je suis Charlie («I am Charlie») quickly spread on media platforms in support of the magazine and the victims. However, few -if not many- people did not support the -entire- slogan and would even claim Je ne suis pas Charlie («I am not Charlie»). Such diverging points of view often led to heated debate -when not arguments- on social media or long discussions between friends. Finding common grounds between these two sides has to be thoroughly scrutinized yet with a view to reaching reconciliation points. The resolution and even positive transformation of this conflict guide this paper. The conflict finds its roots in satire, the controversial tool used by Charlie Hebdo in its weekly issues. The sustained imbalance of power between different cultures residing in France also fuels the conflict. The right to freedom of expression and the value of laïcité have been claimed so far in support of the newspaper in France. Although such views barely find support around the world, no solution has been suggested to date with a view to finding reconciliation. This contribution will apply approaches taken from peace studies to bridge the diverging positions emanating from 'apparently' conflicting identities. Indeed, the need for dialogue and cultural diversity

1. The author wants to thank Nikita Reece for copyediting this paper before it was submitted for evaluation. 
ought to be promoted through education and communication to lower the affective filter that hampers mutual understanding.

Keywords: Charlie Hebdo, dialogue, identity, freedom of expression, satire, imbalance of power, mediation, peace

\section{Introduction}

The attacks on 7th January 2015 at the headquarters of the satirical magazine Charlie Hebdo in Paris sparked a wave of French and worldwide support for the victims. It also spawned high controversy towards the content released by Charlie Hebdo that led to the deadly assault (Shabi 2016). The slogan Je suis Charlie («I am Charlie») quickly spread on media platforms in solidarity for the magazine and the victims. Those who did not support this slogan would not display or post such a phrase publicly, and would even claim Je ne suis pas Charlie («I am not Charlie»). Despite living in the UK at the time of the attacks, as a French citizen I obviously was affected by such an event. I felt sadness for the loss of human lives and was tormented by the ongoing debate over being or not being Charlie. I thoroughly questioned and discussed the subject and then started to challenge my own position on the matter. Such «cleavage» on being Charlie or not (Leone 2015) created an internal conflict triggered by an outside conflict.

The aim of this paper is to find paths to bridge the gap between those who claim to be Charlie and those who do not. This endeavor to find common grounds sadly has not received much consideration by any side or by neutral third parties (Shabi 2016). This paper will demonstrate how the conflict of positions «to be Charlie» or «not to be Charlie» is not intractable. Dialogue and understanding can help bridge two diverging positions with legitimate -moralinterests. The analysis of interests, needs, and values of each side and of the French context will help deconstruct the conflict to propose potential solutions for the disagreement.

A diagnosis of the issues of the conflict will first be undertaken. It will bring to light the means used by Charlie Hebdo to convey its views: satire and freedom of expression. Then, the positions and legitimate interests of the two sides will be 
critically analyzed. Subsequently, it will be demonstrated how the French cultural meaning and value of laïcité and the imbalance of power respectively shape contrasting visions. Approaches from peace studies will then be considered to bridge the positions. Theories of conflict resolution and conflict transformation will be employed. Negotiation and mediation techniques will be applied so both parties can comprehend their diverging point of views. An ultimate point will be devoted to examining if Charlie Hebdo was an effective tool to build positive peace, which represents the epitomy of integration into human society (Galtung 1964).

\section{Diagnosing the issues}

\section{Apparent issues}

Conflict between «I am Charlie» and «I am not Charlie» partisans is based on the assumptions, perceptions, and understanding of the concepts of satire and freedom of expression. In the matter at hand, two sides can be identified whose comprehension of these notions differs and this lies at the heart of a clash between their stances. Indeed, the position taken on one matter is only the tip of the iceberg. It shows a small part from a larger set of interests which are ultimately based on some -fundamental- needs, deriving from -intouchable- values.

According to Maguire (2015), «satire involves the use of humor to ridicule and shame people or institutions. It's a potent tool for exposing society's ills, especially when it comes to politicians and other powerful people». The word "powerful» here is key in understanding how satire should be used as an effective way to criticize society's vices. Satire is often used towards politics, sex, and religion. The former is often represented by individuals in positions of power and deals with a topic -politics- that doesn't refer to the deep identity of a person. Therefore, political satire can be considered as good satire. Politicians or political parties only represent one's interest and not one's needs. Essentially, this does not clash with any identity value, hence it is less likely to provoke hostilities. Charlie Hebdo's satirical identity will be considered as established. As such, because of the attack it endured, satire will be scrutinized to determine its suitability as a means to raise societal and fundamentalist religious issues at that time and place -multicultural France in a globalized world. 
Article 19 of the 1948 Universal Declaration of Human Rights states that everyone has the right to freedom of opinion and expression -including the freedom to hold opinions without interference- and to seek, receive, and impart information and ideas through any media and regardless of frontiers. In absolute terms, any interference -violent or not- to Charlie Hebdo's work should be condemned accordingly. However, as the saying goes, «one's freedom ends where another's begins»:

The right to freedom of expression is a qualified right: it allows expression that might «offend, shock or disturb» but prohibits «insults», «abusive attacks» and «hate speech». [...] I argue that all cartoons of the Prophet Muhammad, which although might offend Muslims, are an acceptable form of expression in Western democracies except cartoon [...] implying the Prophet Muhammad as a «terrorist' which is «insulting» and "an abusive attack» on the Muslim community and Islam. (Shah 2017)

This statement raises an important issue. Was Charlie Hebdo's intent to insult, attack or hate the Prophet? No, says Luz, one of Charlie Hebdo's drawers. He points out that the journal «has always sought to break taboos and shatter symbols and every possible [type of] fanatism» (VICE News 2015). However, the issue here is how, why, and to what extent the caricatures did offend Muslims -perceiving themselves as victims of the drawings- and provoked a discomfort within other parts of the world, and France itself. French laws qualify freedom of expression by providing measures against defamation, insult, and provocation to hatred. To understand the effect Charlie Hebdo's drawings had, the bigger «scope and scale» (Dawes 2015) to which the cartoons were disseminated and how it was understood by its direct and indirect audience must be taken into account.

\section{Structural issues}

To take position on being Charlie or not, as said earlier, reflects certain interests to which one is attached to (Fisher, Ury, and Patton 1991). Besides, the importance of an individual's needs -and values- should not be underestimated. Indeed, these need to be fulfilled -and respected-for that individual to feel 
well (Rosenberg 2003). Positions and interests being the consequence of those needs, understanding the needs of "the other» seems to be the way to connect diverging positions and interests. In the case of the position of «I am Charlie», perhaps what needs clarifying is its -hidden- meaning.

As Maussen (2015) states, the identification that went with the slogan «I am Charlie» signified both solidarity with the victims and support for the "principle of free speech». This argumentation was mainstream within the French general population and led to the 1.6 million people who attended the march following the attack (The Guardian 2015). However, within and especially outside France, some people refused or could not agree to saying, «I am Charlie». Indeed, despite being committed to defend the freedom of speech against such attacks, they felt uncomfortable with Charlie Hebdo's abstract use of that freedom (Hamburger 2017).

Examining the points of view of three of Charlie Hebdo's members of staff regarding the slogan «I am Charlie» may help to legitimize its meaning. Their opinion would take away the sensationalized signification which drove debates in social media and critics of the people claiming that they were not Charlie (Dawes 2015). Indeed, Charlie Hebdo magazine was propelled to the status of celebrity after the attacks. Already banned in the 1970s for three years, it had low numbers of subscribers before the attacks (Weston 2006). It then suddenly became a byword for freedom of expression and an incarnation of traditional republican values. Gérard Biard, editor-in-chief of Charlie Hebdo, said it better when stating that people affirmed to be Charlie without having any idea of what Charlie actually was (Evans and Biard 2017). Zineb El Rhazoui, Charlie Hebdo's journalist during the killings, and still a member of the staffing team, considers being Charlie Hebdo as being willing «to die because of a drawing, because of his own ideas and because of a certain idea of freedom [...] and not everyone has the balls to die for his ideas, so not everyone can be Charlie Hebdo» (The University of Chicago 2015). This slightly radical opinion suggests that saying «I am Charlie» is bona fide to only a limited number of people: Charlie Hebdo's staff, journalists, and activists alike, or also those who already died for such cause. Such clarification of meaning is absent when she omits to clarify that it is only the extremist and fanatic part of Islam that amounts to a dogma, not Islam itself. According to Luz, religions' dogmas are often interpreted and influenced by «rabbis, vicars, mullah -people interpreting others' faith to serve political issues, not always peaceful ones» (VICE News 2015). 
The omission of a clear-cut clarification of the wording and of the meaning of the controversial drawings of the Muslim Prophet is an illustration of the affective filter impeding mutual understanding. Working under the French law and not the Sharia-law argument that El Rhazoui reinforces appears to be a weak argument considering the globalized time in which the event occurred. Indeed, the rapid development of new technologies, media, and information surpasses the slower integration of different people in France. These people come from cultures who approach concepts differently than what might be taken for granted. As such, this globalization era makes it challenging to contain the spreading of information -which may offend someone. This was also refered to as tensions between the local -France- and the global -world- (Weston Vauclair 2015).

Thus, how could Charlie Hebdo magazine have balanced its intrinsic nature, its values, when the subject of the conflict was the impractical and subjective matter of interpretation and taste? Such subjectivity hinders the establishment of an ethical standard. Besides, boundaries will always shift (Somers 2015), depending on the capacity of the victims to react positively or negatively. This interpretation issue is what can perhaps classify Charlie Hebdo as doing "good» or «bad» satire. The former criticizes power and the powerful whilst the latter deliberately hurts and smears the identities of the already marginalized and oppressed people.

This «bad» satire flourishes because of the imbalance of power in France between the non-threatened, privileged and secure majority versus a threatened and insecure minority: the white, French, Christian influenced majority versus the multi-ethnic, pluri-national, Muslim community. The Muslim community in France is the largest of the European Union and there is no doubt as to its marginalization (Trench 2016). Such imbalance of power in the French context is embedded with Catholicism being the dominant religion. Islam is the faith of a marginalized and often immigrant population. Indeed, there is a difference in caricaturing the Pope in the traditionally Catholic France and caricaturing the Prophet Muhammad when Muslims are an oppressed minority: «Satire of Catholicism has a different meaning in a country whose modern political traditions arose largely out of a struggle with and against the Church than satire of the religion of that country's former colonial subjects» (Hamburger 2017). In the current structural and cultural context of France, satire may thus have the effect of sustaining the hegemony from the colonial past.

To understand from another point of view the divergence of positions between the two sides, it is also necessary to clarify the meaning of laïcité and 
the cultural context of French satirical humor. Vulgar humor comes from a long French tradition of satirical newspapers (Weston 2006). This type of journalism was born during the French Revolution at a time when people did not know how to read and write. Besides, it was easier to share and spread information by making cartoons. The inappropriate humor of Charlie Hebdo thus dates back to the critique of religions from the Enlightenment time of Rabelais and Voltaire. The term laïcité was generated then. It is often translated in English as secularism. However, there is a difference between the two words. According to Tarhan (2011), «secularism is usually described as more tolerant towards public visibility of religion; a secular state plays a passive role and allows religious symbols in the public domain. In laicism the state plays a more active role by excluding religious symbols from the public domain and thus confines religion to the private domain». Zineb El Rhazoui exemplifies this difference when she states the most important way to integrate in France is to leave your religious identity at home: "when you are outside you are a French citizen and you are a Muslim at home if you want». Taking this opinion, it would amount to some sort of repression and, consequently, a form of structural violence (Galtung and Ikeda 1995). Identity is part of oneself whether one is outside or at home. Therefore, religion is often part of one's deep identity. Hence, it makes it impractical if not hypocritical to ask for separation. In this sense, laïcité is at the basis of a «double-standard» of freedom of expression (Trench 2016). Laïcité being a specific French feature of its culture, this «lost in translation» issue may be at the root cause of the disagreement. Therefore, it is not surprising to note that freedom of speech is not approached in the same way in the French and the Anglo-Saxon world, despite Biard asserting that even if laïcité is very French it is still a universal value (Evans and Biard 2017).

\section{Bridging diverging positions from peace studies}

In the aim of bridging diverging stances and finding posible uniting solutions, it has been important to exude the interests of each "party» focusing on their values and needs. Such analysis will allow finding connecting points between the two positions «I am Charlie» and «I am not Charlie». Indeed, as Fisher, Ury, and Patton (1991) explained, unwise agreements are reached when it is bargained over positions while less attention is devoted to deconstructing the deeper 
concerns of the parties. A conflict resolution perspective is the approach that will be taken next by trying to generate a variety of possibilities (Fisher, Ury, and Patton 1991). It will be used from the above analysis of the various arguments and the underlying structural issues emanating from it. The people involved will be separated from the problem. The focus will be made on their interests, not their position. Even though debate and negotiations over positions or even over interests to arrive at a final solution may seem here too much content-centered with a focus on the result (Lederach 2003), it is a first step, a first attempt to resolve the indifferences.

Nevertheless, the main goal is to deconstruct the affective filter that may hinder the full comprehension of the meaning behind «I am Charlie» or «I am not Charlie». The signification of the content released by the satirical magazine must be clearly interpreted and explained because the source of conflict lies in the misinterpretation. For instance, because satire is not something that has to please people and make one feel uncomfortable, it is of high importance to ensure that the message that has been delivered is fully understood for acknowledgement and acceptance. Comprehension of meaning and absence of misinterpretation is fundamental at the interpersonal level: between people with a close relationship. It should also be required at the structural and cultural level, when institutions and entities deliver information to a large local, national, or international audience.

Therefore, clarification of the meaning of the delivered content by the sender to the receiver is key in avoiding misunderstanding and conflict. However, seeing how Charlie Hebdo was threatened for years, then maybe some issues still remain. The satirical means used by Charlie Hebdo or the inability by the receiver to understand the means or, surely, both are to be clarified and improved. Indeed, as Gérard Biart pointed out, «we live in a world of images and basically very few people know how to read an image, especially a press drawing as it isn't an illustration of a fact of actuality, but of a context, a perspective and especially an opinion. [...] People's interpretation of cartoons, or what they want to see in, usually don't have anything to do with what was meant» (Evans and Biard 2017). Besides, the interpretative work needed to understand a cartoon's critical message is a work that not everyone is willing or able to do (Hamburger 2017). In today's globalization era, the problem is that what is written in a place can be read, deformed, interpreted at the other end of the world. In this case, opinion journalism will always probably shock someone. In this sense, Fisher, Ury, and Patton's (1991) fourth negotiation method insisting that the result be based on 
some objective standard cannot be exercised. Indeed, we cannot be objective since as human beings we are inherently subjective subjects:

The arbitrator deals largely with the objective; the mediator, the subjective. The arbitrator is generally a passive functionary who determines right or wrong; the mediator is generally an active functionary who attempts to move the parties to reconciliation and agreement, regardless of who or what is right or wrong. [...] Mediation is used where there is a reasonable likelihood that the parties will be able to reach an agreement with the assistance of a neutral. Usually, mediation is used when parties will have an ongoing relationship after resolution of the conflict. (Fahey and Armstrong 1992)

To bridge diverging opinions, we thus ought to be active mediators. To do so, perceptions and assumptions must be overcome. Putting oneself in the shoes of the other to «understand empathetically the power of their point of view and to feel the emotional force with which they believe in it» (Fisher, Ury, and Patton 1991) is essential. Trying to see the situation from all angles helps in building bridges between diverging positions. I realised that behind opposed positions lied «shared and compatible interests, as well as conflicting ones» (Fisher, Ury, and Patton 1991). One of the main solution to dismantle the affective filter is asking «what do you mean?» and «why?».

As «communication is never an easy thing, even between people who have enormous background of shared values and experience» (Fisher, Ury, and Patton 1991), dialogue over debate and transformation over resolution should be preferred in the attempt to find common grounds. «Resolution implies finding a solution to a problem. [...] Transformation directs us toward change, to how things move from one shape to a different one» (Lederach 2003). Lederach, here, prompts for the creation and construction of something new to move from conflict to peace. Dialogue, here, is the peaceful tool. «Successful social movements aren't built on bandwagons and hashtags, but through genuine dialogue» (Balaji 2016). Taking the view of John Lederach (2003) that «rather than seeing peace as a static "end-state", conflict transformation views peace as a continuously evolving and developing quality of relationship», it may be time to have a social revolution in France, fifty years after the May 1968 protests events, a peaceful revolution advocating for dialogue, mutual understanding, and equity between the diverse present cultures in France. As conflict impacts us personally, relationally, structurally, and culturally (Lederach 2003), changes 
must be stimulated through transformation of conflict at various level. Means of mindfulness at the personal level, increased quality of dialogue at the relational level, improvement of institutional entities at the structural level and understanding of diverse identities at the cultural level are some examples. «Inner dialogue, in person space, also known as meditation, to clarify one's own attitudes and assumptions» (Galtung 1996) is, nevertheless, firstly required at the personal level before taking it to the above three «outer dialogues, in social space» (Galtung 1996) for conflict transformation. Such peace-making, embedding the actors in a new formation and transforming attitudes and assumptions (Galtung 1996) could be taught with the South-African Ubuntu philosophy, «I am because we are». It is interesting to note that questioning identity issues within a culturally hegemonic society is what may foster, or certainly be the root of (Lederach 2003), disagreements and conflicts in France. Indeed, the imbalance of power and the impossibility for people to truly understand the satirical means reflect bigger structural and cultural issues that would need to be dealt with. People with hyphenated identities may be the ones who have the mediation skills to catalyze mutual understanding and reconciliation (Lederach 1999). For others, forgiveness is then perhaps a first step towards reconciliation: mutual forgiveness between the people who hurt and the ones who got hurt. Indeed, the problem is not the individual but the criticized ideology, which both parties despised. Thus, «the majority of ordinary people of every faith, race and colour, should stand together to these extremists and say enough is enough» (The Guardian 2015), perhaps by replacing «I am Charlie» or «I am not Charlie» to «I am sorry». This seemingly better slogan would allow a better common identification from both sides. It is therefore time to move from debates and criticisms to dialogue. In other words, to move from a state of negative peace to a state of positive peace, as Galtung (1964) says: to move from the only absence of violence or war to the integration of human society.

Finally, analyzing the extent to which Charlie Hebdo was building positive peace is equivalent to delving into and understanding the core element that constitute the magazine: satire. It is also a way to critically analyze from a peace studies point of view such a concept. Satire is defined as a way of criticizing people or ideas in a humorous way. However, the following comments on satire show that it has a negative essence:

Satire is intrinsically dangerous [...] its provocations have to be handled with care as well as cleverness, a kind of sensationalist journalism intended to stir up 
a hornet's nest, [...] "Satire is negative", -John Mullan- And that's the point... Great satire wouldn't get written if there wasn't something wrong to write about, [...] it's as rife with hate, snobbery and injustice as it is with the finer, higher virtues of hypocrisy-bashing. [...] The purpose is not to create social change but to prevent it. (Allemang 2015).

Satire needs to cause offense. It must. That is its function. (Somers 2015)

The fact is that a lot of what self-consciously passes for satire is mean-spirited and hateful. (Maguire 2015)

Satire is inherently iconoclastic, mocking what is serious, stating plainly what is taboo, and profaning what is sacred. (Hamburger 2017)

Gérard Biart's comments on satire then reaffirm its nature and what Charlie Hebdo was trying to do through it: «to disturb, attract the eye, illuminate by giving a sort of slap [...] The satire does not have to be respectful». The dangerous, offensive, and disrespectful attributes linked to satire thus demonstrates that it is a tool to build negative peace. Indeed, it only aims to stop something undesirable happening and does not promote positive content such as restoration of relationships, the creation of social systems that serve the needs of the whole population, and the constructive transformation of conflict, as positive peace does (Dijkema 2007).

\section{Conclusion}

After the 2015 attacks of Charlie Hebdo in January and those of Paris in November, followed by those of Nice in July 2016, responses by the French government were principally militaristic -whether through the reinforcement of the state of emergency or military attacks on terrorism (McGrogan 2017). Such attempts managed to build negative peace at best or stir more conflict for political ends at worst. The French government means of war against terrorism having no -effective- strategy, drawings of Charlie Hebdo may have endeavored to tackle terrorism based on fundamentalists ideology. However, it did not thrive in the French structural and cultural context of that time. The identity problem, 
where cohesion and identity tend to form within increasingly narrower lines than those that encompass national citizenship (Lederach 1999), undoubtedly is today's main French sickness. Charlie Hebdo failed to address it positively. Past assimilation and integration processes addressed by French institutions to model its society (Seidman 2006) may need to move to hyphenation and promote the acceptance and celebration of hyphenated identities. This move would be logical in the current era of globalization to acknowledge the diversity of French society on a truer level. Assimilation dates back to the time of colonization and integration may refer more to the efforts that first -or second-generation of migrants had to make. Today, French citizens from the third generation -and after- of migrants and the ones who have had the chance to experiment another culture for a very long time might feel as part of various identities. Identities which, if culturally contrasting, might tear the individual inside. This recognition of cultural differences within a same individual, which could constitute a threat to the Republican value of French identity (McGrogan 2017) is a necessity that has not been taken into account yet by the French government but that would however certainly be one of the ways to foster positive peace.

Nevertheless, the means for a long-term positive outcome is to promote quality dialogue between people having diverging positions regarding Charlie Hebdo's content. Peace education and fostering the culture of peace in France must be developed to achieve this goal.

\section{References}

Allemang, John. 2015. «Satire is often nasty, harmful and grotesquely abusive». The Global and Mail. https://www.theglobeandmail.com/opinion/satire-isoften-nasty-harmful-and-grotesquely-abusive/article22389814/.

Balaji, Murali. 2016. «Why facilitating dialogue is more challenging than ever». HuffingtonPost.https://www.huffingtonpost.com/murali-balaji/why-facilitatingdialogue_b_8933220.html.

Dawes, Simon. 2015. «Charlie Hebdo, free speech and counter speech». Sociological Research Online 20 (3): 3.

Dijkema, Claske. 2007. «Negative versus positive peace». Irenees.net. http://www. irenees.net/bdf_fiche-notions-186_en.html. 
Evans, Ryan and Gérard Biard. 2017. «Satire, religion, and terror: A conversation with the Editor-In-Chief of Charlie Hebdo». War on the Rocks. https:// warontherocks.com/2017/12/satire-terror-conversation-editor-chief-charliehebdo/.

Fahey, Joseph J. and Richard Armstrong. 1992. A Peace Reader. Essential Readings on War, Justice, Non-Violence and World Order. Revised Ed. Mahwah: Paulist Press.

Fisher, Roger, William Ury, and Bruce Patton. 1991. Getting to Yes, Negotiating Agreement Without Giving In. 2nd ed. New York: Penguin Books.

Galtung, Johan. 1964. «An editorial». Journal of Peace Research 1 (1): 1-4.

Galtung, Johan. 1996. Peace by Peaceful Means. London: SAGE.

Galtung, Johan and Daisaku Ikeda. 1995. Choose Peace. London: Pluto Press.

Hamburger, Jacob. 2017. "What Charlie Hebdo taught me about freedom of speech». Los Angeles Review of Books. https://lareviewofbooks.org/article/ what-charlie-hebdo-taught-me-about-freedom-of-speech/.

Lederach, John Paul. 1999. Building Peace: Sustainable Reconciliation in Divided Societies. Washington: United States Institute of Peace.

Lederach, John Paul. 2003. The Little Book of Conflict Transformation. Intercourse: Good Books.

Leone, Massimo. 2015. «To be or not to be Charlie Hebdo: Ritual patterns of opinion formation in the social networks.» Social Semiotics 25 (5): 656-680.

Maguire, Laura. 2015. «The power and perils of satire». Philosophy Talks. https:// www.philosophytalk.org/blog/power-and-perils-satire.

Maussen, Marcel. 2015. «Why 'Charlie Hebdo' is not about free speech». CritCom. http://critcom.councilforeuropeanstudies.org/why-charlie-hebdo-is-notabout-free-speech/.

McGrogan, Manus. 2017. "Charlie Hebdo: The poverty of satire». Jacobin Mag. https://www.jacobinmag.com/2017/01/charlie-hebdo-satire-islamophobialaicite-terrorism-free-speech/.

Rosenberg, Marshall B. 2003. Nonviolent Communication: A Language of Life. Encinitas: PuddleDancer Press. 2nd Ed.

Seidman, Michael. 2006. «Inmigraciones en Francia: una perspectiva histórica». Revista de Libros 118: 3-6

Shabi, Rachel. 2016. "Charlie Hebdo free speech debate: Nobody is listening». Al Jazeera. https://www.aljazeera.com/indepth/opinion/2016/01/closed-debatefree-speech-charlie-hebdo-france-muslims-160114083733533.html. 
Shah, Niaz A. 2017. «Charlie Hebdo: Testing the limits of freedom of expression». Muslim World Journal of Human Rights 14 (1): 83-112.

Somers, Erin. 2015. "Satire and the question of taste». The Ploughshares Blog. http://blog.pshares.org/index.php/satire-and-the-question-of-taste/.

Tarhan, Gulce. 2011. «Roots of the headscarf debate: Laicism and secularism in France and Turkey». Journal of Political Inquiry 4: 1-17.

The Guardian. 2015. "Charlie Hebdo and the defence and definition of free speech». The Guardian. https://www.theguardian.com/world/2015/jan/08/ charlie-hebdo-defence-definition-free-speech.

The Guardian. 2015. «Charlie Hebdo attacks: Je ne suis pas Charlie - I am not Charlie | Guardian Docs cartoonist Luz». https://www.youtube.com/ watch?v=u6EzlBkQ910.

The University of Chicago. 2015. «Who Is Charlie? Charlie Hebdo journalist Zineb El Rhazoui on freedom of expression». https://www.youtube.com/ watch?v=MXCMA2DtRXQ.

Trench, Brian. 2016. "Charlie Hebdo", islamophobia and freedoms of the Press». Studies: An Irish Quarterly Review 105 (418): 183-191.

VICE News. 2015. «Exclusive interview with 'Charlie Hebdo' cartoonist Luz». https://www.youtube.com/watch?v=ebL1oCy6tgY.

Weston, Jane. 2006. «Charlie Hebdo and joyful resistance». In Laughter and Power, edited by John Phillips and John Parkin, 209-241. Bern: Peter Lang.

Weston Vauclair, Jane. 2015. «Local laughter, global polemics: Understanding Charlie Hebdo». European Comic Art 8 (1): 6, 9. 


\title{
In defense of the Republic: La Vanguardia's plea for multicultural unity in the Spanish Civil War
}

\author{
Samantha N. Edwards \\ sne23@cam.ac.uk
}

Barcelona's most prominent newspaper, La Vanguardia, used its pages to construct a discursive challenge to fascism and its allies during the Spanish Civil War even when it became clear defeat was imminent. To this end, the newspaper sought to forge a national imaginary -a fraternal link between Catalonia and the rest of Spain- through a reinterpretation of Spain's contentious national history. Editorials prioritised the sieges of sites and monuments of cultural prestige over the Catalanist movements coursing throughout the region, re-contextualising a divisive past as a contemporary symbol for national liberation and unity. La Vanguardia served as an active participant in a war of words unleashed by the violent clash between irreconcilable ideologies, at once defending the popularly-elected government and reimagining a multicultural nation for readers at home and abroad. The upheaval following the military coup of 1936 catapulted Spain to international headlines, making participants aware of a global audience to entice and persuade. Drawing upon Benedict Anderson's concept of imagined communities and George Schöpflin's analysis of national mythmaking in relation to Ruth Wodak's approach to historical discourse, this essay uses textual analysis to illuminate the ways in which a major newspaper framed a unified Spanish community from a discordant past in the hopes of ensuring a shared Republican future. It subsequently analyses the unique position of a Spanish-language newspaper operating in Barcelona during the 
Spanish Civil War, the ongoing dialogue of what constitutes national identity in Spain, and the critical role(s) assumed by the press in wartime.

Keywords: La Vanguardia, Spanish Civil War, discourse analysis, national myth-making, nationalism

\section{Introduction}

Barcelona's most prominent newspaper, La Vanguardia, used its pages to construct a discursive challenge to fascism and its allies during the Spanish Civil War even when it became clear defeat was imminent. Its contributors made a desperate plea for democratic values, not just to imperiled locals or to the international community they still sought to recruit, but to future generations of Spanish citizens. In a striking break with the past, La Vanguardia extended its headline to designate itself «al servicio de la democracia» [at the service of democracy] on September 10, 1936, arguing for the newfound rights of women, minorities, workers, and Catalans. The themes crafted by La Vanguardia reveal a telling aspect of the Republican political discourse, that of complete faith in a revolutionary future which guaranteed justice and liberty for a people still to come. This process involved the unification of a multicultural country to combat a common enemy actively working to impose Castilian hegemony and stamp out the national pride, language, and culture of other regions.

Communication was no easy task as Barcelona represented, at the time, an extreme example of Republican disorder. Riots erupted on a regular basis, mobs ran rampant, and an atmosphere of suspicion overwhelmed the city. Radical socialists challenged less-radical socialists, communists attacked anarchists, and anarchists criticised liberals and progressives, so that Barcelona gained a reputation for chaos. Strikes periodically disrupted operations, leading to the infamous «May Days» of 1937, during which rival factions viciously fought one another in the streets for control of the local government in a series of «violent confrontations across the city» (Graham 2002, 270; Romero Salvadó 2005, 139142; Esenwein and Shubert 1995, 217-224). To this end, the newspaper sought to build a national imaginary -a fraternal link between Catalonia and the rest of Spain- through a reinterpretation of Spain's contentious national history. 
Editorials prioritised the sieges of sites and monuments of cultural prestige over the Catalanist movements coursing throughout the region, re-contextualising a divisive past as a contemporary symbol for national liberation and unity. Faber, quoting Preston, has stated: «the military conflict in Spain gave rise to a 'war of words' [...] a discursive battle that was fought within Spain as much as outside it» (Faber 2008, 215). La Vanguardia was an active participant in this "war of words», at once defending the popularly-elected government and reimagining a multicultural nation for readers at home and abroad.

Following the work of Wodak et al. $(2009,24,31)$, I interrogate this national imaginary as a «founding myth» which attempts to establish the roots of a nation in the «time before time» to consolidate members of a group. National identities are the result of a «discursive construction» that aims to impose a sense of order upon the surrounding world (ibid, 1-6). The upheaval of the military coup in 1936 catapulted Spain to international headlines, making participants aware of a global audience to entice and persuade (Bordería Ortiz 2017, 254). Though located on opposite sides of the political spectrum, both pro-Republicans and pro-Nationalists condensed their representations of the Spanish Civil War into a two-dimensional struggle "characterised on the left as an encounter between the forces of fascism and democracy and on the right as one between Christian civilisation and communist barbarism» (Esenwein and Shubert 1995, 2). Drawing upon Benedict Anderson's concept of imagined communities and George Schöpflin's analysis of national myth-making in relation to Ruth Wodak's approach to historical discourse, I use textual analysis to illuminate the ways in which a Republican newspaper attempted to construct a unified Spanish community from a discordant past in the hopes of ensuring a shared Republican future. This essay traces La Vanguardia's creation of a national imaginary in which all the regions of Spain formed a unified anti-fascist state. I analyse the unique position of a Spanish-language newspaper operating in Barcelona during the Spanish Civil War, the ongoing dialogue of what constitutes national identity in Spain, and the critical role(s) assumed by the press in wartime.

Newspapers play a prominent role in the construction and dissemination of national histories and identities as they explain, consciously and unconsciously, the news they report. Consciously, they may favour their benefactors or political affiliations. Unconsciously, they may highlight or subvert prejudices and ideologies. As Wodak et al. $(2009,24)$ argue, national identity implies «a complex of similar conceptions and perceptual schemata, of similar emotional dispositions and attitudes, and of similar behavioural conventions, which bearers 
of this 'national identity' share collectively and which they have internalised through socialisation». They contend that because «history always relates to the present and the future», then «we cannot understand the future without making references to certain pasts». Schöpflin asserts that myths function as a method for collectivities, especially nations, «to establish and determine the boundaries of their own being, their own systems of morality and values» (Schöpflin 1997, 19-20). He defines myths as «a set of beliefs, usually put forth as a narrative, held by a community about itself». Even when the artificial roots of narratives appear evident, they do not necessarily diminish their persuasive power as myths relate to the so-called essence of a group and conception of self. National identities are thus the discursive constructs that come to define communities and differentiate them from one another, and are all the more significant for their reliance on fictional origins.

La Vanguardia's editorials responded to a series of issues threatening Barcelona's survival in wartime, including the destructive confrontations between socialist and anarchist unions and the rise of separatist movements. It positioned Barcelona at the centre of conflict by cultivating the image of a unified city racing toward victory and summarised the war as an age-old battle between two forces and two ideologies: the foreign invaders against the Spanish, the old regime against the new Republican order (Romero Salvadó 2005, 29). By maintaining the newspaper's tradition of publishing in Castilian in a Catalan city despite radical changes in tone and leadership, La Vanguardia issued a powerful message of solidarity with Republican authorities. It engaged a diverse readership through the promotion of a common language, perhaps in the attempt to transform an ancient symbol of imperial hegemony into a positive tool for unity and inclusivity. In light of these challenges, this essay examines how La Vanguardia forged the notion of a common history in an ethnically and linguistically diverse nation by framing Spain as a multicultural but unified entity, the Second Spanish Republic its noble protector.

\section{«Pensemos en Bilbao»: Consolidating National Narratives of Resistance}

As Basque Country fell piece by piece to Nationalist aggression, La Vanguardia printed a series of headlines and editorials claiming a «spiritual link» between Basques and Catalans, spinning their storied pasts of resistance 
in the face of adversity as proof of cultural affinity. ${ }^{2}$ Between March and July 1937, the newspaper bolstered conceptions of an inclusive (or consolidated) Spanish national identity by highlighting historically marginalised nationalisms -Basques, Catalans, Valencians, and Galicians- as the unique and venerable cornerstones of Spain. It thus conceived of a varied but communal Spanish character, impressing upon readers the central Republican narrative of a legitimate and undivided nation. In one editorial published three days after the bombing of Guernica, La Vanguardia (April 29, 1937) forged a fraternal connection between Barcelona and Bilbao. By incorporating the name «Euzkadi», («Euzkadi, en su lucha por la libertad y la independencia, necesita el apoyo de Cataluña. ¡No lo olvidéis, catalanes!») [Euzkadi, in its fight for liberty and independence, needs Catalonia's help. Don't forget it, Catalans!], La Vanguardia empathised with Republican allies in Northern Spain. Arguing for cooperation among the warring factions of Barcelona while also focusing on the «viejos caminos de simpatía» [old bonds of sympathy] between Catalonia and the Basque Country, La Vanguardia advocated for a multicultural society drawn together by a common history and held together by the shared investment in a better future.

Multiculturalism responds to the premise of the coexistence of multiple national or cultural groups occupying a space with overlapping boundaries. Generally, certain groups ascend dominance over others, using myth-making "to establish the sole way of ordering the world and defining world-views» and asserting that holding a monopoly is vital to the sustained existence of a community (Schöpflin 1997, 20). Multiculturalists reject this view, arguing in favour of the cultural enrichment of diversity. For centuries, Castilian Spain overshadowed peripheral territories, leading to a renewed decentralist movement in the founding of the Second Spanish Republic which had committed to the recognition of regional languages, expanded cultural liberties, and limited selfrule. To this end, the editorial promoted a sense of kinship between the Basque and Catalan capitals by describing them as fundamental pillars of Spain, Spanish history, and Spanish identity. «Mutuamente atraídas»[mutually attracted], Barcelona and Bilbao were positioned as the two poles of Spanish life:

2. For more examples, see the following editorials in La Vanguardia: «Por la libertad de Euzkadi». May 14, 1937; «Los voluntarios». May 22, 1937; «La bárbara destrucción de Guernica». May 26, 1937; «Viva Euzkadi!». June 22, 1937. 
Las dos ciudades representan los dos puntos extremos de un eje en torno al cual se han desarrollado los principales acontecimientos de la vida española. Esta similitud de destino, puede más que las diferencias que las separan, y se ha puesto desde la sublevación acá más en evidencia que nunca. (La Vanguardia April 29, 1937)

[The two cities represent the two ends of an axis around which the fundamental events of Spanish life have developed. This similitude of destiny is greater than the differences that separate them, and, since the insurrection, has become more evident than ever before.]

This overt dual positioning of Bilbao and Barcelona as iconic Basque and Catalan cities, respectively, but also as being integral to Spanish life, reflected La Vanguardia's (re)imagining of a Spanish identity inclusive of Basque and Catalan nationalisms. The editorial envisioned a cohesive Spanish state in which diverse groups fought together against «el fascismo internacional». In so doing, it promoted the representation of the Spanish Civil War as the eruption of an international conflict on a national stage, in which «true» Spaniards defended Spain against an outside invasion, and related the heroics of Madrid on May 2, 1808 to those of Bilbao on May 2, 1874.

1808 marked a pivotal year as Spaniards retaliated against the French occupying forces, engaging in guerrilla warfare tactics and eventually reclaiming Spain's independence in 1814. Commonly cited as the first conscious articulation of Spanish nationalism, 1808 is often remembered as the beginning of the eventually victorious Spanish struggle against foreign oppression (Blinkhorn 1975, 5; Núñez Seixas 2005, 52):

Alguna vez hemos dicho, cuando al hablar de la defensa de Madrid se apelaba al recuerdo del dos de mayo de 1808, que nos parecía el ejemplo inoportuno. El pueblo de Madrid fue heroico en aquella jornada trágica, pero vencido y sacrificado por las tropas invasoras... Bilbao celebra también su dos de mayo pero ésta es verdadera fiesta de júbilo. Recuerda el dos de mayo de 1874, día en que las tropas carlistas levantaron el cerco que habían puesto a la villa- aquel día ganó el tercer entorchado y conquistó el título de invicta[...] La invicta villa lo será una vez más. (La Vanguardia April 29, 1937)

[We said once, when the defence of Madrid appealed to the memory of the 2nd of May, 1808, that it seemed to us an ill-chosen example. The people of Madrid were heroic on that tragic day, but they were defeated and sacrificed by the invading troops... Bilbao also celebrates the 2nd of May but for them it is a truly festive holiday. It commemorates the 2nd of May, 1874, the day the Carlist troops lifted their siege of the city-the day they earned their third aiguillette and won the title of indomitable[...] The indomitable city shall be so once again.] 
La Vanguardia's celebration of «la invicta villa» [the indominable city] demonstrated a strategic focus on Bilbao's historic stance against Carlism, a rightist and insular political ideology which remained popular in parts of the Basque Country, especially Navarra (Holguin 2007, 28). To this effect, the editorial's throwback to May 2, 1874 recalls the defence of the First Spanish Republic (1873-1874) and the fight against the restoration of the Bourbon monarchy in the Third Carlist War. Bilbao successfully held off attackers for four months. A Basque publication commemorating the event in 1887 even defined the confrontation as part of «la eterna lucha entre la libertad y el despotismo, entre la tiranía religiosa y el sacratísimo derecho de conciencia» [the eternal fight between liberty and despotism, between religious tyranny and most sacred right of conscience] (de Alcíbar 1887, 6). In weaving together the most celebrated examples of resistance of Bilbao and Madrid -despite the first being «glorious» and the latter at once «tragic» and «heroic»- the editorial likens them to a single battle for self-determination against tyranny. This theme would have resonated with Basque and Catalan readers, many of whom supported at least some degree of regional autonomy.

Indirectly addressing the recent bombing of Guernica, which marked »a crucial turning point in the northern offensive» against the Republic (Phillips and Phillips 2010,255), the editorial attempted the ambitious feat of unifying a diverse readership whose members pertained to a variety of social classes, ethnic groups, and political affiliations. Above all, La Vanguardia called upon readers to be active participants in the conflict, reminding them of the Basque Country's geographic importance: «Bilbao corre riesgo grave y Bilbao es uno de los núcleos vitales de la República en armas» [Bilbao is at serious risk and Bilbao is one of the vital nuclei of the Republic in arms]. Indeed, the editorial consists of numerous reminders: «Bilbao atraviesa días de evidente peligro. Sería insensatez negarlo y pueril esconderlo» [Bilbao is experiencing days of clear danger. It would be foolish to deny it and puerile to conceal it], «Bilbao está en peligro» [Bilbao is in danger], and «Se ayuda a Bilbao recordando que en Aragón existe un frente de batalla y haciéndoselo recordar a quien sea el que lo ha olvidado» [Bilbao can be helped by remembering that a battle front lies in Aragon and reminding whosoever has forgotten about it]. It stressed the dangers of losing ground in the north, especially as it opened up another line of attack against Catalonia. 
Its literary style commonly oscillated between romantic rhetoric and blunt inflection, at once emphasizing the dangers of a Basque defeat and bolstering public moral with patriotic discourse:

Tres veces llegaron frente a ella, en el pasado siglo, las fuerzas enemigas de la libertad, y tres veces tuvieron que levantar el sitio vencidas. Bilbao no conoce el significado de la palabra rendición. Entre las gestas ciudadanas que nos sirven de ejemplos históricos estimulantes, las de Bilbao son las mejores. En ellas, el valor y la fortaleza, fueron recompensados con la victoria.

[Three times in the last century have the enemy forces of liberty stood before her, and three times have they been forced to lift their siege defeated. Bilbao does not know the meaning of the word 'surrender'. Among the civilian movements to serve as inspiring historic reminders, Bilbao's are the best. In them, valour and strength were rewarded with victory.]

In idealising the «tradición gloriosa» [glorious tradition] of «la invicta villa», La Vanguardia guided readers through a sanctioned Republican narrative interpretation of the war and warned against a civilian passivity deemed fatal. Political narratives, like all narratives, are primarily composed of four parts: characters, setting, plot, and theme (Rowland 2005, 136). These components are born out of the political discourse of the era and are packaged for mass consumption in a series of interpretive frames which serve to make sense of the otherwise elusive human experience. To this effect, the editorial highlighted the virtues of the protagonist (Bilbao) over the weaknesses of the antagonist (fascism). In so doing, Bilbao transcends its status as the «eternal» symbol of Basque nationalism to become a beacon of liberty and independence for the whole of the Second Spanish Republic.

The Fascist Offensive in the Basque Country, sometimes called the «War in the North», commenced in late March, 1937. Within a month Republican forces were on the retreat and suffering continuous setbacks. The destruction of Guernica, the historic Basque capital, which left hundreds dead, dealt a devastating blow to the region. Home to an ancient oak, under which the Basque fueros, or code of laws, were proclaimed according to a supposedly democratic tradition stretching back into legend, Guernica had long been upheld as emblematic of Basque society and government. By the twentieth century it was considered a minor settlement, but its destruction shocked the Western world (Phillips and Phillips 2010, 255-256). Still more disturbing was the realisation 
that the loss of Basque lands signaled Catalonia's weakening position as it came under direct fire from insurgent forces.

Though the modern Basque and Catalan nationalist movements emerged contemporaneously, they assumed different characteristics. «Both regions were overdeveloped and characterized by similar levels of ethnic competition», but Basque nationalism tended toward total independence from Spain and adherence to Catholicism, while Catalanism generally involved regional autonomy as part of a federation of Spanish states which aimed to limit the centralised power of Madrid (Díez Medrano 1994, 541, 543). Catalonia underwent heavy industrialisation and dominated approximately $80 \%$ of Spain's textile market. In the process it developed an advanced political and economic infrastructure well-adapted to capitalist interests (ibid, 553). The Basque Country underwent similar levels of industrial transformation, becoming the leading exporter of iron ore, but its development was distributed unevenly and to the detriment of the lower classes who suffered from harmful mining and agrarian reforms (ibid, 555; Shubert 2005, 27). The «separatist and reactionary character of Basque nationalism» thus «differed dramatically from the pro-capitalist and generally non-separatist character of Catalan nationalism» of the period as their respective leaders prioritised divergent values and policies (Díez Medrano 1994, 543).

Unlike Catalan nationalism, Basque nationalism donned a largely conservative tone, in some areas joining with Carlist ideologies. While Carlism had previously ignored external affairs, exerting its efforts into stamping out the outside influences of liberalism and secularism, five years under the Second Spanish Republic apparently awakened it to the development of a «worldwide political crisis, in which left and right were seen to be hurtling towards a collision, crushing democracy between them». According to Blinkhorn, Carlist reporting and commentary on foreign affairs constituted a thoroughly propagandistic function of promoting public fear of all left-wing victories, to advance the stimulating triumphs of rightist movements throughout the continent, and most importantly, «to advertise Carlism as the vanguard of a similar triumph in Spain» (Blinkhorn 1975, 141).

Though the Pact of San Sebastián (1930) had promised autonomy to Catalonia, Galicia, and the Basque Country, the actual implementation of statutes proved a more difficult task, with some officials supporting the motion and others insisting that «Spain was an indivisible country» (Holguin 2007, 29; Hernández Lafuente 1980, 35-42). The specifics of regional autonomy emerged as a critical point of contention in the nascent Republican administration, revealing the 
deep ambivalence of the Spanish left regarding the nature of power-relations and multiculturalism within Spain. This angered the Catalan nationalists who had played a key role in the establishment of the Second Spanish Republic, who felt betrayed as proposals consistently stalled in federal legislatures. Indeed, the debate over the implementation of a centralised or confederate Spanish state remained a major source of disagreement among the diverse and highly stratified network of political parties governing Spain. The inability to reconcile the competing priorities of Madrid and Barcelona further strained regional relations, increasing nationalist demands for independence. Tensions reached an all-time high when in 1934 Companys announced the «Estat Català» after he found his campaign for agrarian reform blocked by Republican deadlock in other parts of the country. Though the announcement was reversed within hours and Companys arrested for treason, the question of separatism continued to influence political affairs on a national level, continually bubbling to the surface in the events leading up to, and during, the Spanish Civil War (Esenwein and Shubert 1995, 55-58; Graham 2002, 218-221).

Catalonia was finally granted limited self-governance in 1934 while the Basque Country was hastily granted a similar agreement in 1936 only after the outbreak of war. The Republic feared Basque anarchist and nationalist groups, citing competition with anarchist strongholds in the north and suspected Nationalist collaboration with the Partido Nacional Vasco (PNV). Consequently, at the same time that La Vanguardia garnered support for Basque allies with impassioned cries of «Pensemos en Bilbao, catalanes» [Let's think about Bilbao, Catalans] (April 29, 1937), the Republic withheld supplies and firearms from the region. La Vanguardia's political imaginary of a united federation of Spanish states against an army consisting of military traitors and hostile foreigners was thus contradicted by actual events, a phenomenon Phillips and Phillips characterise as demonstrative of the complexities of the Spanish Civil War as «Insiders experienced the surreal complexity of hatreds and multi-layered loyalties and animosities» (Phillips and Phillips 2010, 256).

Despite La Vanguardia's narrative of indomitable Basque resistance, Bilbao surrendered to Franco without a fight in June of 1937, fuelling Republican suspicions of clandestine cooperation between the Nationalists and PNV. Franco's capture of the Basque Country opened up a second battlefront against Catalonia, culminating in the disastrous Battle of the Ebro the following year (ibid, 256-257). La Vanguardia endeavoured, therefore, to cultivate a space in which individuals of various cultural backgrounds could interact, fortify 
a collective national defence, and effectively battle injustice in a cause which became increasingly desperate. ${ }^{3}$ Its spirited depiction of Bilbao tied Basques and Catalans to a single destiny, suggesting they made up two sides of the same coin, or more precisely, that standing alone meant dying alone. Such odes to Basque courage and heritage were evidently intended mobilise readers and prepare Barcelona for the fast-approaching "Batalla de Cataluña». The forging of an epic destiny linking Bilbao and Barcelona to one other and to the rest of Spain likely sought to stabilise a city divided by workers' strikes, factional schisms, irreconcilable political ideologies, and clashing conceptions of what constituted the fabric of Spanish society.

\section{Conclusion}

La Vanguardia survives today as Catalonia's leading newspaper and boasts the fourth highest readership in Spain. The iconic title aptly describes its fierce resistance to Franco throughout the Spanish Civil War, operating as a staunch defender of the Second Spanish Republic until the fall of Barcelona and publishing six days a week to motivate and inform a diverse population. Furthermore, it did so for a time under the direction of María Luz Morales Godoy, the first woman ever to head a Spanish newspaper of national circulation (Cabré 2017, 27; Amiguet 2015). Throughout the war, La Vanguardia constructed a new national imaginary for a unified Spanish resistance to military insurgents. Every citizen -Castilian, Catalan, Valencian, Galician, or Basque- was made out to be essential to a shared Republican victory. Even as battles were waged and lost, territories conquered, and tens of thousands of refugees displaced, $\mathrm{La}$ Vanguardia stood behind the popularly-elected government in declaring that a sustained collective resistance in Catalonia could save Spain and the rest of Europe from the clutches of fascism.

On March 15, 1938, La Vanguardia's editorial featured the famous Catalan phrase: «Ara és l'hora, catalans!» [Now is the time, Catalans!], which maintains its association with Catalanist movements. Its exact origins remain uncertain, but as the official chant of the National Day of Catalonia celebrated on September

3. La Vanguardia continued to develop this theme of an indomitable people with respect to the island of Menorca in June, 1938. See «Menorca, la indómita», La Vanguardia, June 30, 1938. 
11 it honours the fallen heroes who defended Barcelona against Philip V in the War of Spanish Succession (1702-1715). The siege, which began in 1713 and lasted fourteen months, resulted in the loss of Catalan liberties, institutions, and laws (Pietschmann 2016, 61-62; Anguera 2003, 17-38). Still remembered as a symbol of the continued Catalan struggle for independence, La Vanguardia transformed a Catalanist narrative into a Republican narrative of joint CatalanSpanish resistance to Spain's fascist tyrants. It began by acknowledging that «la guerra que estaba lejos, muy lejos de Cataluña» [the war that was far away, so far away from Catalonia] now rapidly approached by way of Aragon. It declared that Madrid felt similarly anxious when the war first breached its walls, but persisted despite the danger: «la realidad cruel vencía a los temperamentos más flemáticos; pero vibró, se estremeció, perdió su calma idiosincrásica y se puso a arder de ira, de rabia, de abnegación, de furor violento». [The cruel reality overcame the most apathetic of temperaments; but it shook and shuddered; cast aside its distinctive calm and began to burn with anger, with rage, with selfdenial, with violent passion.] In so doing, it reiterated the fraternal connection between Madrid and Barcelona, urging Catalans to emulate their heroism.

Preserving the connection between the two capitals by following in Madrid's example was necessary, it argued, for the protection of the Catalan language and culture threatened by the Nationalist agenda:

¿Se han dado cuenta los catalanes de lo que significaría para ellos el triunfo, de las hordas que avanzan por Aragón? Nada más que esto: supresión de todos los periódicos catalanes; desaparición, en tiendas, librerías, bibliotecas públicas y privadas, de todos los libros escritos en lengua catalana; persecución de la bandera barrada; abolición de las emisiones catalanas por radio: prohibición de hablar catalán en público. (La Vanguardia March 15, 1937)

[Do Catalans realise what the triumph of the hordes currently advancing through Aragon would mean for them? Nothing short of this: suppression of all Catalan newspapers; disappearance of all books written in Catalan from our shops, bookstores, public and private libraries; persecution of our striped flag; abolishment of radio broadcasts in Catalan; bans on speaking Catalan in public.]

The «bandera barrada» [striped flag] referred, of course, to the Senyera, which prevails as an important symbol of Catalan pride (Olivieri 2015). The editorial adopted an uncharacteristically Catalan nationalist tone that spoke directly to Catalonia, but which still furthered the newspaper's argument for 
a collective national imaginary: «Hablamos de lo puramente catalán, porque a los catalanes nos dirigimos» [We speak of the purely Catalan because we address the Catalans]. La Vanguardia stirred Catalan nationalism to promote a regional resistance connected to a broader sense of Spanish resistance rooted in nationalist discourse. It argued that the Second Spanish Republic fought for the preservation of democracy in all parts of Spain against Franco who posed an unprecedented threat to Catalonia's heritage: «Ara es l'hora, catalans-como dice el himno de la tierra- ara és l'hora d'estar alerta [...]

La personalidad de Cataluña está en litigio como no lo estuvo nunca en su historia. En las manos de sus hijos reside [...]» [Now is the time, Catalans -in the words of our nation's hymn- now is the time to be alert [...] The spirit of Catalonia is in dispute like never before in our history [...] Her fate rests in the hands of all her children [...]]. Such rallying cries to protect «la patria» contended that the Republic fought to defend a Spanish homeland inclusive of Catalonia and other historically marginalised national groups, while the fascists fought to conquer Spain and destroy Catalan identity completely.

In this way, La Vanguardia kept alive the Spanish Revolution of 1930, the progressive reforms implemented by the legitimate government, and the Catalan thirst for regional autonomy and recognition as a distinct cultural centre of Spain. The Second Spanish Republic had implemented radical legislation outlining universal suffrage, public education, divorce by mutual consent, freedom of speech and of assembly, the disestablishment of the Jesuit order, the right to autonomy for Catalonia, Galicia, and the Basque Country (though drawn up, the statutes for the autonomy of Valencia were never finalised), and promises of land redistribution. Payne (1993, 60-61) described the Republic as Spain's «first complete experience with modern democracy» at a time when European affairs made a «dramatic» turn toward authoritarianism and dictatorship. The war against fascism, as it was commonly categorised, transcended regional borders to become the decisive battle «ipor la vida!» [for life!], "por el amor a la tierra y el amor a la libertad» [for love of land and liberty] (La Vanguardia April 10, 1938), and for the future benefit of Spain's most marginalised populations.

The military uprising of July 19, 1936 resulted in the government seizure of La Vanguardia (July 22, 1936) and marked the initiation of a distinctly leftist and pro-republican stance which it upheld until the fall of Barcelona in January, 1939. The Godó family -its founders and former owners- fled to Italy in 1936, where they remained until the end of the war (Dalmau 2019, 192). It was at this time they renamed the periodical La Vanguardia Española [The 
Spanish Vanguard] in line with the monolithic conception of Spanish national identity promoted by Franco (La Vanguardia January 27, 1939). While Franco's Nationalists sought to establish Spain as a homogenous country defined by absolute allegiance to Catholicism and, above all, to Castilian hegemony, Barcelona's La Vanguardia envisioned Spain as a multicultural nation unified by Republican ideals guaranteeing the protection of national liberties. As Esenwein and Shubert $(1995,34)$ somewhat controversially maintain, it was not Republican revolutionary failure but rather Republican revolutionary success which prompted the ultimately fatal military rebellion of July, 1936, for it was «motivated by fears that the Republic would be successful in in its wide-ranging reforms, the end result of which would have been to replace one economic, social, and political formation -or one vision of 'Spain'- with another». Consequently, the famous «war of words» (Preston 1984, 1) unleashed by the Spanish Civil War reflected a discursive battle between competing narratives of Spanish identity. By framing the history of Spain's multiple nations as a single campaign for justice and liberty, La Vanguardia served the Republican mission to recast Spain as a modern and progressive child of the democratic revolution.

\section{References}

Alcíbar, Miguel de. 1887. "Bilbao y el periódico La Guerra». Dos de Mayo, $13^{\circ}$ Aniversário, Sociedad «El Sitio»: 6. http://www.bilbao.eus/bld/imagenesbld/00 786229/000/00786229.pdf

Amiguet, Teresa. 2015. «María Luz Morales, el periodismo tiene nombre de mujer». La Vanguardia. September 22, 2015.

Anguera, Pere. 2003. «El 11 de septiembre: Orígenes y consolidación de la Diada». Ayer 51: 17-38.

Anderson, Benedict. 1996. Imagined Communities: Reflections on the Origin and Spread of Nationalism, Revised Edition. London: Verso.

Blinkhorn, Martin. 1975. Carlism and Crisis in Spain, 1931-1939. Cambridge: Cambridge University Press.

Bordería Ortiz, Enrique. 2017. «Premsa, propaganda i vida quotidiana a València (1936-1937)». In València: Capital de la República 1936-1937, vol. 2, edited by Javier Navarro and Sergio Valero, 253-275. Valencia: Ajuntament de València. 
Cabré, María Ángeles. 2017. María Luz Morales, pionera del periodismo. Barcelona: La Vanguardia Ediciones.

Dalmau, Pol. 2019. Press, Politics, and National Identities in Catalonia: The Transformation of La Vanguardia, 1881-1931. Brighton: Sussex Academic Press.

Díez Medrano, Juan. 1994. «Patterns of development and nationalism: Basque and Catalan nationalism before the Spanish Civil War». Theory and Society 23.4: 541-569.

Esdaile, Charles J. 2019. The Spanish Civil War: A Military History. London: Routledge.

Esenwein, George, and Adrian Shubert. 1995. Spain at War: The Spanish Civil War in Context, 1931-1939. London and New York: Longman.

Faber, Sebastiaan. 2008. Anglo-American Hispanists and the Spanish Civil War: Hispanophilia, Commitment, and Discipline. New York: Palgrave Macmillan.

Graham, Helen. 2002. The Spanish Republic at War, 1936-1939. Cambridge: Cambridge University Press.

Hernández Lafuente, Adolfo. 1980. Autonomía e integración en la Segunda República. Madrid: Ediciones Encuentro.

Holguín, Sandie. 2007. «Navigating the historical labyrinth of the Spanish Civil War». In Teaching Representations of the Spanish Civil War, edited by Noël Valis, 23-32. New York: Modern Language Association of America.

La Vanguardia. 1937. «La bárbara destrucción de Guernica». May 26.

La Vanguardia. 1937. «Los voluntarios». May 22.

La Vanguardia. 1937. «Pensemos en Bilbao, catalanes». April 29.

La Vanguardia. 1937. «Por la libertad de Euzkadi». May 14.

La Vanguardia. 1937. «iViva Euzkadi!» June 22.

La Vanguardia. 1938. «Ara és l'hora, catalans!» March 15.

La Vanguardia. 1938. «iPor la vida!» April 10.

Núñez Seixas, Xosé-Manoel. 2005. «Nations in arms against the invader: on nationalist discourses during the Spanish Civil War». In The Splintering of Spain: Cultural History and the Spanish Civil War, 1936-1939, edited by Chris Ealham and Michael Richards, 45-67. Cambridge: Cambridge University Press.

Olivieri, Victor M. 2015. «Sub-state nationalism in Spain: Primers and triggers of identity politics in Catalonia and the Basque Country». Ethnic and Racial Studies 38 (9): 1-17. 
Payne, Stanley G. 1993. Spain's First Democracy: The Second Republic, 1931-1936. Madison: The University of Wisconsin Press.

Phillips, Jr., William D., and Carla Rahn Phillips. 2010. A Concise History of Spain. Cambridge: Cambridge University Press.

Pietschmann, Horst. 2016. «Barcelona, Catalonia and the Crown of Aragón in the Bourbon Spanish Empire». European Review 25 (1): 60-68.

Preston, Paul. 1989. The Spanish Civil War: 1936-1939. Chicago: The Dorsey Press.

Preston, Paul. ed. 1984. Revolution and War in Spain, 1931-1939. London and New York: Routledge.

Romero Salvadó, Francisco J. 2005. The Spanish Civil War: Origins, Course and Outcomes. New York: Palgrave Macmillan.

Rowland, Robert. 2005. «The narrative perspective». In The Art of Rhetorical Criticism, edited by Jim A. Kuypers, 125-146. Boston: Pearson Education, Inc. Schöpflin, George. 1997. «The functions of myth and a taxonomy of myths». In Myths and Nationhood, edited by Geoffrey Hosking and George Schöpflin, 1935. London: Hurst \& Company.

Shubert, Adrian. 2005. A Social History of Modern Spain. London and New York: Routledge.

Wodak, Ruth, Rudolph de Cillia, Martin Riesigl, and Karin Liebhart, eds. 2009. The Discursive Construction of National Identity, 2nd Edition. Edinburgh: Edinburgh University Press. 


\title{
La justicia transicional en perspectiva: los casos de Argentina, Colombia y Sudáfrica
}

\author{
Camilo Eduardo Espinosa Díaz \\ camiloespinosa10@gmail.com
}

El dilema entre justicia y verdad representa una paradoja presente en los juicios más importantes de la historia reciente de la humanidad, como Núremberg, Tokio y los tribunales ad hoc de la ex Yugoslavia y Ruanda. La pregunta de investigación que aborda este capítulo es, teniendo en cuenta el acuerdo de paz de 2016 firmado por el Estado colombiano y el grupo guerrillero Farc, qué puede aprender este país de la experiencia de los Juicios a las Juntas Militares en Argentina y de la Comisión de la Verdad y la Reconciliación en Sudáfrica en relación con los conceptos de justicia y verdad. La experiencia en Colombia muestra un camino intermedio, en el que se tomaron decisiones basadas en la experiencia de Sudáfrica, como darles participación a las víctimas; y también en la de Argentina, con la administración de justicia para todos los actores, incluidos terceros, que participaron en el conflicto armado.

Palabras clave: justicia transicional, verdad, paz, conflicto armado, Colombia 


\section{Introducción}

La justicia transicional es el esfuerzo que hace una sociedad para superar regímenes déspotas y tiránicos o guerras civiles, e instaurar un orden democrático y pacífico de largo aliento. Durante este esfuerzo, se presentan dilemas y paradojas difíciles de resolver. Por un lado, están las víctimas, que tienen el derecho a saber qué fue lo que pasó, a que esos hechos no vuelvan a ocurrir, a la reparación y a la justicia. Por otro, los victimarios, quienes poseen información instrumental para la realización de esos derechos pero que, durante los procesos de negociación, buscan amnistías o indultos. Lo que muestra la justicia transicional es el debate entre justicia y verdad (o paz, en términos más amplios). ¿Cómo lograr un equilibrio sin que se rompa el frágil tejido social de un país o unas negociaciones de paz? Para el caso colombiano, estos dilemas se acentúan porque no hubo transición (Uprimny Yepes et al. 2006), pues el conflicto armado persiste, aunque sea de baja intensidad. La experiencia de otros países, como Argentina y Sudáfrica, puede servir a Colombia para extraer lecciones aprendidas e inspirarse en las mejores prácticas de cada caso para construir una sociedad sostenible.

Lo que muestra la experiencia argentina y sudafricana son aprendizajes diametralmente opuestos: en la primera, la balanza se inclinó a favor de la resolución judicial del conflicto, pues fue poca la contribución desde los perpetradores al esclarecimiento de la verdad (Nino 1997; Hilb 2010); en la segunda, todos los actores del conflicto étnico tuvieron voz y participación proporcional, y los mecanismos creados motivaron a los victimarios a contar lo sucedido (Hilb 2010). Así, fueron los criminales de este país africano los más interesados en decir lo que había ocurrido (Cassin 2004), lo cual generó un clima más favorable para la reconciliación, pues se puso en el centro el interés común de todas las partes el producir la verdad más amplia posible (Hilb 2010).

Este artículo busca contribuir a la discusión sobre la pertinencia de la justicia transicional en este país. El interés en realizar esta investigación estriba en dos argumentos fundamentales vinculados al diálogo entre distintas experiencias. En primer lugar, se trata de un tema de actualidad que polariza en Colombia (Villalobos 2017; Piccone 2019) y del que poco se ha escrito desde una mirada de lecciones aprendidas entre estos tres países (Panjor y Heemmina 2018). En segundo lugar, desde una perspectiva comparada, los casos de Argentina y Sudáfrica ofrecen pistas de cómo abordar los temas de verdad y justicia en 
Colombia. En cuanto a la metodología, el artículo es de corte cualitativo y emplea el método comparativo de las ciencias sociales. Las técnicas de investigación usadas son el análisis bibliográfico y el análisis documental.

El artículo está organizado de la siguiente manera: en primer lugar, se aborda los conceptos de justicia y verdad en los casos de Argentina y Sudáfrica; seguidamente, se hace un análisis sustancial del punto cinco de los acuerdos firmados por el Estado colombiano y las Farc, sobre víctimas y justicia. Finalmente, se plantean los resultados y las conclusiones. A manera de abrebocas, cabe destacar que el caso colombiano, que es pionero a nivel mundial (Sanz Luque 2016; Santos 2019), logra un acuerdo que busca el cierre definitivo del conflicto armado jurídicamente pero también estructuralmente (véase Galtung 1969; 1998), y privilegia la verdad y la justicia.

\section{Dos casos paradigmáticos para mirar con espejo retrovisor: Argentina y Sudáfrica}

A continuación, se abordan dos casos emblemáticos sobre la materia de la justicia transicional, que ponen en contraste los principios de justicia y verdad. Esta revisión se elabora a partir de las publicaciones académicas. El primero de los casos es el Juicio a las Juntas Militares en Argentina en 1985.

El pasado de masivas violaciones contra los derechos humanos durante la dictadura argentina finalizó con la llegada de la democracia. En 1983, el presidente Alfonsín ordenó la creación de la Comisión Nacional sobre la Desaparición de Personas, que estuvo compuesta por distinguidas personalidades académicas y culturales de ese país. ${ }^{1}$ Sin entrar en el detalle de los hechos ocurridos en Argentina durante la dictadura, la opción que eligió dicho país fue una justicia que sacrificó la verdad (Hilb 2011). El Juicio a las Juntas culminó con la condena a prisión perpetua de dos de los nueve inculpados, condenas menores para otros tres y la absolución de cuatro de ellos. ${ }^{2}$

1. Se estima que en nueve meses de operación, dicha Comisión logró recopilar testimonios de más de nueve mil desapariciones, la organización de campos de concentración y el modo operativo de la política de desaparición, tortura y asesinato (Hilb 2010). El trabajo de esta Comisión quedó consignado en el informe Nunca más (Comisión Nacional sobre la Desaparición de Personas (CONADEP) 1984).

2. «Los Comandantes en Jefe del Ejército y de la Armada, Jorge Videla y Eduardo Massera, integrantes de la primera Junta Militar, fueron condenados a prisión perpetua, el Brigadier Agosti, coman- 
Lo que se critica de la elección argentina (Vezzetti 2002; Hilb 2010, 2011) es que la opción de justicia no dio lugar a la verdad, es decir, al esclarecimiento de los hechos y la identificación del paradero de las personas dadas por desaparecidas. En otras palabras, no se pudo acceder a la verdad desde los perpetradores, los militares. No se le dio un espacio al victimario para contribuir, desde el relato, a aquello que pasó. La justicia a la argentina creó un incentivo, el incentivo del silencio, en donde decir la verdad era equivalente a una larga condena en prisión.

Cabe mencionar que, en el caso argentino, con el propósito de limitar la duración de los juicios a las Juntas Militares, se crearon leyes de punto final y de obediencia debida, que fueron aprobadas por el Congreso de Argentina en 1987. Dieciocho años después, el mismo Congreso las declaró inexequibles, decisión que fue secundada por la Suprema Corte del país.

Por otra parte, en el caso sudafricano, se optó por otra vía para hacer el tránsito del régimen del apartheid a la democracia. La decisión que tomó la sociedad fue la verdad, en contrapartida de la justicia. En ese país se conformó la Comisión de la Verdad y la Reconciliación, que escuchó en igual proporción a víctimas y victimarios. La fórmula fue la siguiente: aquellas personas que se consideraban víctimas y comparecían ante la Comisión serían inicialmente escuchadas y podrían obtener, eventualmente, una reparación; por su parte, los perpetradores que, de manera voluntaria (esto es importante señalarlo), se presentaran ante esta entidad dentro de un periodo previamente establecido y contaran el qué, el cómo, el cuándo y el dónde de lo que pasó, serían amnistiados, con una única salvedad: que sus actos y hechos estuvieran asociados a un objetivo político. ${ }^{3}$

Quien no solicitara la amnistía y fuera posteriormente inculpado por una denuncia, o quien la solicitara y no procediera a lo que, a juicio de la Comisión, era un relato exhaustivo, proseguiría el camino de la justicia ordinaria. Todas las

dante de la Fuerza Área e integrantes de esa misma Junta recibió una condena de cuatro años y medio. Todos ellos fueran asimismo inhabilitados para el ejercicio de toda función pública. También fueron condenados e inhabilitados para toda función pública el General Viola (17 años) y el Almirante Lambruschini (8 años), dos de los tres integrantes de la segunda Junta y resultaron absueltos el Brigadier Grafigna (Fuerza Área, segunda Junta), Galtieri, Anaya y Lami Dozo (tercera Junta). Tras apelación, la Corta Suprema redujo posteriormente ligeramente las condenas de Viola y Agosti» (Hilb 2010, 11).

3. La amnistía comprendía los actos cometidos entre el 1 de marzo de 1960 y el 10 de mayo de 1994, es decir, un periodo de 34 años. 
graves violaciones de derechos humanos, cualquiera fuera su actor o el campo al que perteneciera eran alcanzadas por las disposiciones (Hilb 2010, 12).

La salida a la sudafricana involucró tanto a los partidarios del apartheid que, con la creación de la nueva Constitución (1993), fue declarado crimen contra la humanidad, como a quienes habían cometido delitos luchando contra este régimen. En contraste con lo que sucedió en Argentina, la versión sudafricana creó el incentivo de la reconciliación, para que los perpetradores confesaran sus crímenes a cambio de beneficios jurídicos, con el fin de obtener la mayor verdad posible de todo lo que ocurrió.

Frente a lo ocurrido en Sudáfrica, cabe destacar que, de las 21.290 víctimas que sometieron sus declaraciones a la Comisión, ésta reconoció a 19.050, a las que se sumaron otras 2.975 identificadas en el curso de su labor. 7.116 perpetradores solicitaron la amnistía y fueron oídos en audiencias públicas. Durante 1.888 días en 267 sitios diferentes, con una cobertura mediática permanente, la población sudafricana pudo conocer, en la voz y en las múltiples lenguas de víctimas y victimarios, las historias más tremendas sucedidas bajo los ojos durante los treinta años precedentes (Hilb 2010, 13).

Otro elemento de contraste entre estos dos casos es la distinta mirada frente a la reconciliación, el arrepentimiento y el perdón. En Argentina, lo anterior fue escasamente posible, por la forma y los dispositivos que se generaron a partir del enjuiciamiento a los militares (Hilb 2010). En Sudáfrica, sin ser categóricos, en algunos casos hubo arrepentimiento y perdón, pero lo que sí es claro es que la transición a la reconciliación fue menos escabrosa (Hilb 2011). La sociedad sudafricana en su conjunto escuchó, de quienes por muchos años habían mantenido el establecimiento, la historia infame de los crímenes del apartheid. Los perpetradores tuvieron que contar toda la historia para no ir a la cárcel, ante la mirada de las víctimas o sus familiares.

\section{La justicia transicional en los acuerdos de La Habana: un análisis sustancial}

El acuerdo sobre qué tipo de justicia aplicar en los diálogos de paz entre el Estado colombiano y las Farc es complejo, no tiene una respuesta única y levanta pasiones y odios (Gómez-Suárez 2016) entre la población colombiana. Lo que se acordó en La Habana (Cuba) no dejó satisfechos a todos y todas. Cuando se 
habla de justicia transicional, que es el modelo propuesto para esta negociación, se espera que la sociedad haga un gran esfuerzo para enfrentar un pasado de masivas violaciones a los derechos humanos y al derecho internacional humanitario, con el propósito de hacer el tránsito de la guerra a la paz o de la dictadura a la democracia (Uprimny Yepes et al. 2006).

Ese modelo intermedio y flexible con los estándares jurídicos internacionales contemporáneos es conocido como justicia transicional y abarca toda la variedad de procesos y mecanismos asociados con los intentos de una sociedad por resolver los problemas derivados de un pasado de abusos a gran escala, a fin de que los responsables rindan cuentas de sus actos, servir a la justicia y lograr la reconciliación (Secretario General de Naciones Unidas 2004, 6).

El punto del Acuerdo que aborda este tema es el número cinco y lleva por nombre «Acuerdo sobre las Víctimas del Conflicto: Sistema Integral de Verdad, Justicia, Reparación y No Repetición, incluyendo la Jurisdicción Especial para la Paz; y Compromiso sobre Derechos Humanos» (Gobierno de Colombia 2016, 124). Ahora, ¿cuáles son los objetivos principales de este punto? Fundamentalmente dos: el primero, satisfacer los derechos de las víctimas a la justicia, la verdad, la reparación y darles garantías de no repetición, es decir, que los hechos violentos no vuelvan a ocurrir. El segundo, cerrar la etapa de conflicto armado y pasar a un estado que podríamos llamar de reconciliación. ${ }^{4}$ Desde una mirada amplia, los objetivos de la justicia transicional son: fortalecer el Estado de derecho, sanar heridas del pasado, avanzar en procesos de reconciliación garantizando los derechos de las víctimas, reducir la impunidad, ${ }^{5}$ erradicar las causas estructurales de los conflictos (Ardila 2004). Este punto del Acuerdo contempla todos esos propósitos.

Para hacer el tránsito de un conflicto armado entre una guerrilla y el Estado existen, normalmente, tres caminos:

1. Utilizar los instrumentos de la justicia ordinaria para someter al «enemigo». Esto implica usar las instancias pertinentes (jueces, juzgados, tribunales supremos) y el código penal. Aquí estamos hablando de que una de las partes en cuestión perdió la guerra, es decir, no hubo lugar para

4. Lo opuesto a la reconciliación son las leyes de cierre o punto final.

5. Este componente también alude al deber que tienen los Estados de perseguir y juzgar crímenes internacionales, por ejemplo, crímenes de guerra y de lesa humanidad. 
la negociación. En el caso de Colombia, las Farc no fueron derrotadas militarmente, de manera que este camino no era viable.

2. El segundo camino es el del perdón y olvido, que fue lo que se hizo en el pasado tanto en Colombia como en el cono Sur (Pizarro Leongómez 2017). Aquí hablamos de amplias amnistías para pasar la página y seguir adelante. $^{6}$

3. Por último, tenemos un modelo intermedio, el de la justicia transicional, ubicado en una frontera borrosa y difusa, en el que se sacrifica alguno de los dos valores en cuestión: la verdad o la justicia.

La pregunta que surge a continuación es: qué se necesita, en el contexto de Colombia y en el marco de la justicia transicional, para que sea legítimo. Utilizar los instrumentos de la justicia ordinaria para una negociación con una guerrilla es incoherente, en el sentido de que ningún grupo guerrillero se someterá a un tribunal sin antes haber perdido en el campo de batalla. De esta manera, lo que hay que plantear (y así se dio en los diálogos en Cuba) es un estándar flexible que hoy existe en el derecho internacional y que sea compatible con los compromisos internacionales de Colombia. En síntesis, se habla del derecho que tienen las víctimas a la verdad, a la reparación, a darles garantías de no repetición y que los responsables de todos los bandos, incluidos terceros (como empresas, por ejemplo), rindan cuentas ante un tribunal. ${ }^{7}$

Antes de entrar en detalle de lo que se acordó en el punto de justicia, es importante mencionar tres elementos fundamentales de la negociación. En el centro del acuerdo se sitúa a las víctimas, que no habían sido tenidas en cuenta en ningún proceso de paz anterior en Colombia (Pizarro Leongómez 2017). En segundo lugar y en consonancia con ello, se les dio participación y voz. Sesenta víctimas (representativas) del conflicto armado en el país viajaron a Cuba a hablar con los victimarios. Así mismo, durante las negociaciones, entre 2012 y 2014, se recibieron un total de 9.306 propuestas (Brett 2017) relacionadas con los puntos de la agenda de negociación.

6. Específicamente en los primeros ocho (8) acuerdos de paz en Colombia.

7. Los principios de la justicia transicional se derivan del Informe Final Acerca de la Cuestión de la Impunidad de los Autores de Violaciones de los Derechos Humanos (Joinet 1997). «En conformidad con este informe se identifican las obligaciones de los Estados en procesos de transición a saber: (1) la satisfacción del derecho a la justicia; (2) la satisfacción del derecho a la verdad; (3) la satisfacción de la reparación del derecho de las víctimas; y (4) la adopción de reformas institucionales y otras garantías de no repetición» (Ardila 2004, 4). 
Lo acordado se puede resumir en cinco puntos:

1. La creación de una comisión para el esclarecimiento de la verdad, la convivencia y la no repetición. Su finalidad es satisfacer los derechos de las víctimas en cuanto a la verdad. Es un mecanismo transitorio y extrajudicial. Por antonomasia, será imparcial e independiente.

2. Se conforma la Unidad para la Búsqueda de Personas Dadas por Desaparecidas. Su función es averiguar el paradero de las personas desaparecidas en el marco del conflicto armado colombiano. Es un organismo humanitario, extrajudicial, que será autónomo e independiente. ¿Cómo realizará esta labor?

[...] recolectará y cruzará la información de bases de datos institucionales y no institucionales, establecerá el universo de personas dadas por desaparecidas, pondrá en marcha planes de búsqueda y exhumaciones, y llevará a cabo procesos de identificación. Al final, entregará a los familiares un informe sobre lo acaecido a la persona desaparecida y, cuando sea posible, hará una entrega digna de los restos. (Oficina de Alto Comisionado para la Paz 2016, 26)

3. Medidas de reparación integral para la construcción de la paz. Se busca fortalecer los mecanismos que ya tiene el Estado para reparar a las víctimas y crear nuevas medidas. Dentro de las medidas se destacan la reparación material a la víctima, el desminado humanitario o el apoyo a la construcción de infraestructura (vías terciarias, por ejemplo).

4. La instauración de la Jurisdicción Especial para la Paz. Es la piedra angular de los acuerdos. Se trata del conjunto de órganos que impartirán justicia. Además, es la garantía del Estado para sancionar los delitos cometidos en el conflicto armado. Aquí es importante hacer dos precisiones. Aquellos que han cometido delitos amnistiables o susceptibles de recibir un tratamiento especial, irán directamente a la Sala de Amnistías o Indultos y a la Sala de Definición de Situaciones Jurídicas. Otro camino tomarán aquellos que cometieron delitos no amnistiables ni susceptibles de recibir un tratamiento especial. El esquema que se acordó fue el siguiente (Oficina de Alto Comisionado para la Paz 2016): 
a. Quienes reconozcan responsabilidad por los actos cometidos en el marco del conflicto armado recibirán sanciones de corte retributivo y restaurativo (realización de obras y reparación del daño causado); tendrán una restricción efectiva de la libertad de entre 5 y 8 años.

b. Quienes con posterioridad reconozcan responsabilidad antes del dictamen de una sentencia recibirán sanciones alternativas e irán a la cárcel; tendrán una pena privativa de la libertad de entre 5 y 8 años.

c. Quienes no reconozcan su responsabilidad en la guerra y sean hallados culpables recibirán sanciones ordinarias, irán a cárcel y tendrán pena privativa de la libertad de entre 15 y 20 años.

Finalmente, es importante mencionar que este punto del Acuerdo buscar cerrar jurídicamente el conflicto armado.

5. Garantías de no repetición. Lo que se busca con esta medida es la implementación y coordinación de los mecanismos del sistema integral, en conjunto con los otros puntos de la agenda de negociación.

En este sistema complejo creado por la negociación, la siguiente pregunta que surge es quiénes participarán en él:

1. Evidentemente, las víctimas, que tendrán lugar en todas las instancias del sistema, que busca, como ya se ha mencionado, satisfacer sus derechos humanos.

2. Guerrilleros. Es un grupo que incluye a todos los militantes de las Farc que se sometan al acuerdo, es decir, que las disidencias no gozarán de los mismos beneficios jurídicos.

3. Agentes del Estado. Se aplicará a los agentes del Estado que hayan cometido delitos en el contexto y en razón del conflicto armado, partiendo del reconocimiento de su condición de garantes y de la presunción de que ejercen de manera legítima el monopolio de las armas (Oficina de Alto Comisionado para la Paz 2016, 25).

4. Paramilitares desmovilizados. El fenómeno del paramilitarismo es conexo a la lucha insurgente. De manera que el sistema buscará conocer a profundidad sobre este fenómeno y resarcir a sus víctimas. 
5. Terceros civiles. Podrán ser llamados terceros civiles que, sin formar parte de los grupos armados ilegales, hayan tenido una relación no coaccionada de financiación o colaboración con éstos y en desarrollo de tal vínculo hayan cometido delitos en el contexto y en razón del conflicto armado (Oficina de Alto Comisionado para la Paz 2016, 25).

Otro elemento importante e implícito en este punto es que reconoce las características políticas del conflicto armado. La justificación de las Farc del levantamiento en armas se da en razón al derecho a la rebelión. Sin embargo, lo que interesa traer a colación aquí es que, al reconocer el carácter político del conflicto, se reconoce a la contraparte como actor e interlocutor político también. En este sentido, la amnistía se da precisamente en el marco de ese delito de rebelión y otros conexos, como el delito de narcotráfico; no obstante, quedan excluidos los delitos atroces, que pasarán por la Jurisdicción Especial de Paz. ${ }^{8}$

Este punto del acuerdo no dejó satisfechos a todos y a todas, y en Colombia levantó muchas pasiones y odios (Gómez-Suárez 2016; Piccone 2019). Sin embargo, si se revisa como forma de superar el conflicto armado, es un acuerdo robusto que, si bien sacrifica un poco de justicia, lo hace en pro de las víctimas, del esclarecimiento de la verdad y de la restitución de sus derechos humanos.

\section{Análisis}

Este artículo es de corte cualitativo y hace uso del método comparativo. Los estudios cualitativos se caracterizan por contrastar la teoría con la evidencia empírica (Maxwell 2013). Tonon de Toscano $(2011,2)$, refiriéndose a la tradición del uso de la comparación en los estudios sociales, destaca que existen tres tipos: el análisis histórico, el análisis estadístico y los estudios cualitativos. En este sentido, sostiene esta autora que la comparación tiene tres consideraciones: «como contexto de justificación y control de hipótesis; como contexto de

8. La rebelión consiste en tomar las armas contra un régimen al que se considera «injusto» para deponerlo e instaurar otro («ijusto?»). Carlos Gaviria, uno de los juristas más importantes de la historia de Colombia, citando a Francisco Carrara, sostenía en 2008 que el delincuente político «ni siquiera es delincuente, el delincuente es un hombre equivocado que en uso de las armas perdió, porque si hubiera ganado no sería delincuente sino gobernante» (Pluralidad Z. 2019). En lo personal, valoro del derecho a la rebelión el derecho a disentir, sin embargo, reprocho inexorablemente el uso de las armas como medio (i)legítimo para ejercerlo. 
descubrimiento y de generación de nuevas hipótesis y como proceso lógico o sistemático» (Tonon de Toscano 2011, 2), que es como se denomina el método comparativo. Este método tiene como fin la búsqueda de similitudes y diferencias entre los objetos investigados (Sartori 2002). Por lo tanto, lo que se compara es aquello que pertenece a la misma especie, clase o género (Tonon de Toscano 2011, 2). Para Fideli (1998), lo que se contrasta son las propiedades de los objetos, en un momento particular y un tiempo definido.

El tema que se aborda en este artículo es el de la justicia transicional y, teniendo en cuenta el conflicto armado que vive Colombia, qué puede aprender este país de las experiencias de los Juicios a las Juntas Militares en Argentina y de la Comisión de la Verdad y de la Reconciliación en Sudáfrica. De acuerdo con los casos que se presentan, los conceptos analizados provienen de distintas disciplinas: la ciencia política (los sistemas de gobierno de cada país y las Constituciones), la historia (los hechos transcurridos en Argentina durante la dictadura, en Sudáfrica en el régimen del apartheid y el conflicto armado en Colombia) y el derecho internacional (la justicia transicional y las guerras civiles). Ahora, los conceptos utilizados van en sintonía con los niveles de abstracción identificados por Sartori (2002): nivel alto, que hace referencia a macro temas, en este caso, la justicia; nivel medio, remite a categorías generales, como la justicia transicional y las guerras civiles; y el nivel bajo, son aquellos que son específicos, como los acuerdos logrados en cada uno de los países.

A través del estudio de los temas y conceptos se pudo crear los ejes de análisis de la investigación, que son:

a) Los objetivos y acuerdos alcanzados en cada uno de los países.

b) El tipo de justicia aplicada y las medidas de verdad, reparación y garantías de no repetición implementadas en cada caso.

c) Las experiencias y lecciones aprendidas en perspectiva de Argentina y Sudáfrica.

En cuanto a la selección de la muestra, las unidades espaciales escogidas fueron Argentina, Colombia y Sudáfrica, y el horizonte de tiempo inicia en 1985, que es cuando tienen lugar los Juicios a las Juntas Militares en Argentina, pasando por 1998, fecha en la cual se entrega el primer volumen del informe de la Comisión de la Verdad y de la Reconciliación de Sudáfrica, y llegando a 
2016, que es cuando el Estado colombiano suscribe los acuerdos de paz con la guerrilla de las Farc.

Para la selección de la muestra se crearon dos reglas: la primera, tomar un marco temporal amplio que permita la comparación entre los casos y Colombia; y la segunda, que los casos escogidos se hayan inclinado a favor de alguna de las dos opciones posibles, es decir, justicia o verdad, de tal forma que se pueda revisar los efectos de estos caminos escogidos por los países. De lo anterior, se desprende la propuesta de Dogan y Pelassy (1996) en la que se destaca que el escogimiento de los objetos a comparar se da por criterios pertinentes y no por el gusto del investigador. También se tomó el planteamiento de Lijphart (1969) que señala que los casos a comparar deben tener características similares y, a su vez, variables distintas que valgan la pena contrastar. En este artículo se revisaron los informes «Nunca más» en Argentina (Comisión Nacional sobre la Desaparición de Personas (CONADEP) 1984), de la Comisión de la Verdad y la Reconciliación en Sudáfrica y el punto 5 del acuerdo de paz firmado por el Estado colombiano y la guerrilla de las Farc.

En relación con las técnicas de investigación, se usaron el análisis bibliográfico y el análisis documental. El análisis bibliográfico se hizo conforme a una lectura selectiva, estratégica y crítica de libros y artículos científicos con el objetivo de obtener el marco teórico, conceptual y los datos relevantes de cada caso.

Finalmente, en cuanto al análisis documental, entendido como el trabajo con documentos escritos que abordan un fenómeno social y que permiten el estudio del pasado (Corbetta 2015), se trabajó con documentos institucionales y de carácter público, producto de las instituciones creadas en cada uno de los casos. Se analizaron y estudiaron los informes sobre la cuestión producidos en Argentina, Colombia y Sudáfrica.

\section{Resultados}

La pregunta de investigación es, teniendo en cuenta el proceso de paz en Colombia, qué puede aprender este país de la experiencia de los Juicios a las Juntas Militares en Argentina y de la Comisión de la Verdad y la Reconciliación en Sudáfrica, en relación con los conceptos de justicia y verdad. En relación con ella, se resaltan cinco resultados concretos: 
1. La vía argentina demostró que, si bien fue eficaz en términos de justicia, pues los máximos responsables de violaciones a los derechos humanos fueron encarcelados, la participación de las víctimas y la construcción de la verdad fueron relegadas a otros espacios (Hilb 2011). Los militares no fueron escuchados, no se les dio voz, de manera que el paradero de cientos de desaparecidos, hasta hoy, sigue siendo un misterio. Así mismo, los mecanismos y dispositivos creados no motivaron a los militares a contar voluntariamente lo que había sucedido. Hablar en este contexto era igual a ir a una cárcel durante muchos años.

2. El caso sudafricano es la otra cara de la moneda. Aquí se escuchó a las víctimas y victimarios, en condiciones de proporcionalidad. Este encuentro de dos mundos, televisado durante más de 5 años, creó unas condiciones más propicias para la reconciliación y para cerrar las viejas heridas de la guerra (Hilb 2011). Aquí en algunos casos hubo arrepentimiento y, en consecuencia, perdón, temas vedados en Argentina (Hilb 2010, 21).

4. Del caso sudafricano, Colombia puede sacar lo mejor. Amplia participación de las víctimas en los procesos de verdad y memoria, es decir, se facilitó el contar qué fue lo que pasó, obviamente, para quien se considere víctima y acuda voluntariamente a la Comisión (para el esclarecimiento de la verdad, la convivencia y la no repetición). Además, hay una ventana de oportunidad en aras de la reconciliación, que es uno de los fundamentos de la justicia transicional, la transmisión de este proceso por los medios de comunicación públicos. Ver a las víctimas contar qué fue lo que les pasó, escuchar a los victimarios decir el por qué y cómo lo hicieron, de cara al país, incluidas sus víctimas y familiares, tendría un efecto terapéutico y de «catarsis colectiva» (Rodríguez Montenegro 2011, 58). Frente a esto, Hilb $(2010,13)$ coincide en afirmar que: «el efecto curativo, rehumanizador, que la exposición de las historias tuvo tanto para las víctimas como para los victimarios, sobre el poder creador de comunidad del discurso, sobre la potencia transformadora por la cual el dispositivo de amnistía convertía un mal moral en un bien político».

4. El Acuerdo de La Habana es susceptible de mejoras, pero en su conjunto, es equilibrado y robusto, si se compara, también, con lo alcanzado en Argentina y Sudáfrica. Contempla medidas importantes de verdad y reparación, en sus diversas formas, para las víctimas. Siendo consecuente con sus compromisos internacionales y los tiempos de hoy, Colombia dejó espacio para la justicia de los máximos responsables.

5. Finalmente, se rescata del acuerdo en Colombia el cierre jurídico del conflicto armado. Para ello, contempla justicia y participación para todas las partes, incluidos terceros, como empresas transnacionales, que estuvieron involucrados. Esto es importante mencionarlo, pues en el caso de Argentina, los militares no tuvieron voz. En Sudáfrica, todos los actores tuvieron participación proporcional para contar lo que había sucedido. 


\section{Conclusiones}

Como primera medida, el Acuerdo de La Habana va más allá de la mirada clásica de la justicia, es decir, de la punitiva, pues opta por el camino de la justicia transicional, que es una ley especial que se da en contextos de guerra civil (o conflictos armados) o de dictaduras. Esta justicia a la colombiana incluye castigo para los máximos responsables, que incluyen pena privativa de la libertad proporcional a los hechos causados, e incentivos para que cuenten toda la verdad posible y para que reparen a las víctimas, incluso con su propio patrimonio.

Colombia toma elementos de Argentina y Sudáfrica, sin embargo, labra su propio camino. Este país eligió una vía intermedia, de justicia para los máximos responsables de crímenes asociados al conflicto armado, y a su vez, puso en el centro del Acuerdo a las víctimas y sus derechos (a la verdad, la justicia, la reparación y la no repetición), y creó dispositivos que incentivan la producción de la más amplia verdad posible. Tanto víctimas como victimarios tendrán la oportunidad de ser escuchados en condiciones de proporcionalidad.

En tercer lugar, la creada Jurisdicción Especial de Paz, como el tribunal de crímenes de guerra de Núremberg, el ad hoc para la ex Yugoslavia, Burundi o Ruanda, no es un tribunal permanente, tiene un tiempo perentorio y busca impartir justicia a quienes participaron en el conflicto armado en Colombia. Esto es importante señalarlo porque no es un tribunal como la Corte Penal Internacional, que sí es permanente. El acuerdo de La Habana cumple con los tres principios fundamentales de la justicia transicional: el derecho a la justicia, el derecho a la verdad, el derecho a la reparación integral. En el fondo lo que busca el acuerdo es el cierre jurídico del conflicto, en el sentido de que todas las partes involucradas asistan al tribunal y aclaren su situación. Desde una manera holística, el acuerdo en su conjunto busca cerrar las causas estructurales de la guerra en Colombia, por ello se plantean reformas al agro, participación política, lucha contra las drogas, entre otras.

Otro elemento importante que hace diferentes los casos de Argentina y Sudáfrica y que puede servir a Colombia es el tema de la reconciliación, el arrepentimiento y el perdón. Siendo consciente del peligro que supone abordar el tema, por sus dimensiones religiosas y morales, y porque se inscriben al ámbito privado, desde una perspectiva política, el perdón es el equivalente del arrepentimiento (Walzer 1994). En aquellos lugares en donde se tiene la conciencia de lo que ocurrió, pero que no debió suceder, es decir, donde hay un arrepentimiento 
genuino, es posible imaginar una escena común de reconciliación. La reflexión para Colombia, basada en los casos de Argentina y Sudáfrica, es que sólo a partir de una apuesta en común de quienes se arrepienten y quienes perdonan, es decir, de ponerse en común, es posible construir un/os relato/s de (re)conciliación/ones. En el caso sudafricano este artilugio está presente, mientras que, en el argentino, no.

Para finalizar, el caso colombiano, que tomó elementos de Argentina y Sudáfrica, la exposición a la/s verdad/es va a ser el elemento integrador y de poner en común el interés de todas las partes por el diálogo, en contrapartida del silencio, que por décadas ha sido el gran protagonista (sin desmerecer los grandes esfuerzos que ha hecho el Centro Nacional de Memoria Histórica - CNMH). Llegó la hora de la/s verdad/es para Colombia.

\section{Bibliografía}

Ardila, Dorys. 2004. «Justicia transicional: principios básicos». Pangea. https:// escolapau.uab.cat/img/programas/derecho/justicia/doc004.pdf.

Brett, Roddy. 2017. «La voz de las víctimas en la negociación: sistematización de una experiencia». Programa de Naciones Unidas para el Desarrollo. https:// www.undp.org/content/dam/colombia/docs/Paz/undp-co-victimas2016ajustado-2017.pdf.

Cassin, Barbara. 2004. "Amnistie et pardon: pour une ligne de partage entre éthique et politique». En Le genre humain: vérité, réconciliation, réparation, editado por Barbara Cassin, Cayla Oliver y Philippe-Joseph Salazar, 37-57. París: Seuil.

Comisión Nacional sobre la Desaparición de Personas (CONADEP). 1984. Informe "Nunca más". Buenos Aires: CONADEP.

Corbetta, Piergiorgio. 2015. La ricerca sociale: metodologia e tecniche. Bologna: Il Mulino.

Dogan, Mattei, y Dominique Pelassy. 1996. How to Compare Nations: Strategies in Comparative Politics. Chatham: Chatham House.

Fideli, Roberto. 1998. La comparazione. Milano: Angeli.

Galtung, Johan. 1969. «Violence, peace, and peace research». Journal of Peace Research 6 (3): 167-191. 
Galtung, Johan. 1998. Tras la violencia, 3R: reconstrucción, reconciliación, resolución. Afrontando los efectos visibles e invisibles de la guerra y la violencia. Bilbao: Gernika Gogoratuz.

Gobierno de Colombia. 2016. «Acuerdo final para la terminación del conflicto y la construcción de una paz estable y duradera». Oficina del Alto Comisionado para la Paz. http://www.altocomisionadoparalapaz.gov.co/procesos-y-conversaciones/Documentos \%20compartidos/24-11-2016NuevoAcuerdoFinal.pdf.

Gómez-Suárez, Andrei. 2016. El triunfo del no: la paradoja emocional detrás del plebiscito. Bogotá: Icono.

Hilb, Claudia. 2010. «La virtud de la justicia y su precio en verdad. Una reflexión sobre los juicios a las Juntas en Argentina, a la luz de la Comisión de la Verdad y la Reconciliación en Sudáfrica». Estudios Sociales 39: 9-23.

Hilb, Claudia. 2011. "Virtuous justice, and its price in truth in post-dictatorial Argentina». African Yearbook of Rhetoric 2 (1): 13-20.

Lijphart, Arend. 1969. Comparative Politics and the Comparative Method. Bruselas: Association Internationale de Science Politique.

Maxwell, Joseph Alex. 2013. Qualitative Research Design: An Interactive Approach. Thousand Oaks: Sage.

Nino, Carlos. 1997. Juicio al mal absoluto. Buenos Aires: Emecé.

Oficina de Alto Comisionado para la Paz. 2016. «El acuerdo final de paz. La oportunidad para construir paz». Bogotá. https://www.refworld.org.es/ docid/5a874f254.html

Panjor, Fareeda, y Anchana Heemmina. 2018. «Chapter 3: Peace process and transitional justice: The comparative study of Mindanao, Colombia, and Thailand's Deep South». Asian Affairs: An American Review 45 (2): 78-97. doi: https://doi. org/10.1080/00927678.2019.1584279.

Piccone, Ted. 2019. Peace with Justice: The Colombian Experience with Transitional Justice. Washington: The Brookings Institution.

Pizarro Leongómez, Eduardo. 2017. Cambiar el futuro: historia de los procesos de paz en Colombia (1981-2016). Bogotá: Penguin Random House.

Pluralidad Z. 2019. Carlos Gaviria y el comisionado de paz de Álvaro Uribe Vélez. YouTube. https://www.youtube.com/watch?v=Nb82D2AiN3s

Rodríguez Montenegro, Gina Paola. 2011. «Los límites del perdón. Notas sobre la justicia transicional en Sudáfrica, Centroamérica y Colombia». Justicia Juris 7 (2): 52-66.

Santos, Juan Manuel. 2019. La batalla por la paz. Barcelona: Península Huellas. 
Sanz Luque, Belén. 2016. «Colombia, un proceso de paz con perspectiva de género». El País, 21 de septiembre. https://elpais.com/internacional/2016/09/21/ colombia/1474418366_503575.html.

Sartori, Giovanni. 2002. La política: lógica y método en las ciencias sociales. México: Fondo de Cultura Económica.

Secretario General de Naciones Unidas. 2004. El Estado de derecho y la justicia de transición en las sociedades que sufren o han sufrido conflictos. Nueva York: Consejo General de Naciones Unidas.

Tonon de Toscano, Graciela. 2011. «La utilización del método comparativo en estudios cualitativos en Ciencia Política y Ciencias Sociales: diseño y desarrollo de una tesis doctoral». Kairos: Revista de temas sociales 15 (27): 1-12.

Uprimny Yepes, Rodrigo, María Paula Saffón Sanín, Catalina Botero Marino, y Esteban Restrepo Saldarriaga. 2006. ¿Justicia transicional sin transición? Verdad, justicia y reparación para Colombia. Bogotá: Centro de Estudios Derecho, Justicia y Sociedad.

Vezzetti, Hugo. 2002. Pasado y presente. Guerra, dictadura y sociedad en la Argentina. Buenos Aires: Siglo XXI.

Villalobos, Joaquín. 2017. Del miedo a la ingobernabilidad. La salvadoreñización de Colombia. Bogotá: Fundación Ideas para la Paz.

Walzer, Michael. 1994. Thick and Thin. Moral Argument at Home and Abroad. Indiana: University of Notre Dame Press. 
ÍNDEX 


\title{
Considering degrowth from a feminist transrational peace perspective
}

\author{
Marisol C. Bock \\ a1295846@uji.es
}

We are facing multiple global interlocking crises in the economic, ecological, and social realms. Sustainable economic degrowth recognizes the hegemonic economic growth paradigm as a key problem. As a solution, it aims to promote a just downscaling of the economy, from interdisciplinary perspectives. While equality is a crucial component for degrowth advocates, the movement lacks an engagement with feminist theory. This work seeks to identify how feminism might enhance and promote the degrowth movement. This study provides a theoretical examination of the fields of degrowth and feminism as well as related areas, from a peace perspective, by engaging in conceptual analysis, deconstruction, and critical thinking. The peace perspective includes a notion of the epistemological shift coined by Martínez Guzmán (2000) and links with transrational peace research by Dietrich (2018). The analysis shows that capitalism is dependent on economic growth, yielding patriarchal features. Feminism, which promotes the abolishment of patriarchy is a heterogeneous movement whereby the pursuit and promotion of individual choices prevails, turning the overall canon of feminism into a neo-liberal market of ideas within the growth paradigm. I propose that a feminism useful for the degrowth context should (1) pursue gender equality, (2) recognize the deep-lying cultural roots of patriarchal violence, (3) consider intersectionality, and (4) have an awareness of the parallels between oppression of women and oppression of nature. From a transrational peace perspective, a parallel between patriarchal features in the economic logic 
based on scarcity and the oppression of women can be seen, considering the significance of feminine and masculine principles as described by adjacent fields of study. In conclusion, degrowth may be enhanced and promoted by adopting a growth-critical ecofeminist, transrational perspective. Open research lines include testing the applicability of such insights in the practical realm.

Keywords: degrowth, feminism, transrational, peace

\section{Introduction}

This essay deals with the sustainable economic degrowth movement, from a feminist and peace studies perspective. ${ }^{1}$ My writing embraces Martínez's epistemological shift (2000), the approaches proposed by Adrienne Rich's politics of location (1985), and Stanley and Wise's work on feminist research (2002) calling for a located first-person researcher that reveals their own academic and personal background. Following this line of transparency, I am a GermanVenezuelan female raised in different countries with Western values, in an intercultural context. My interest lies in complex global problems and long-term societal change, upon which I wish to critically and systemically reflect, starting at a grassroots level. I identify as a peace worker, researcher, and feminist, as I seek to trace connections of the personal and the political (Millett 2014) as well as the theoretical and activist side of peace work (Lederach 2004).

My research focuses on the degrowth movement. I understand the necessity for peace work to unfold from the inside out, that is, from the personal to the collective and from the local to the global. My tenets are:

1. This text is based on my master's dissertation «Considering Sustainable Economic Degrowth from a Feminist, Transrational Perspective» in Peace, Conflict and Development Studies (Bock 2015), which serves as the foundation of my current research on the same topic as part of the $\mathrm{PhD}$ program at the UnEsCo Chair of Philosophy for Peace, Universitat Jaume I. 
1) There are several global interlocking crises, in the economic, ecological, and social realms;

2) degrowth directly addresses the root problem underlying these crises -the pursuit of infinite economic growth on a finite planet- and proposes a «democratically led redistributive downscaling of production and consumption in industrialized countries as a means to achieve environmental sustainability, social justice and well-being» (Demaria et al. 2013, 3).

While social equality is an essential component for degrowth advocates, the concern for gender equality has hitherto remained at the margin of degrowth discourse and only a small proportion of degrowth publications adopt a feminist stance. ${ }^{2}$ Against this background, I pose the following question: How can feminism enhance and promote the degrowth movement?

This essay presents a theoretical analysis of the overlapping fields of degrowth and feminism from a peace perspective by engaging in conceptual analysis, deconstruction, and critical thinking. The peace perspective included is linked to the notion of 'transrational peace' (Dietrich 2018) and the 'epistemological shift' (Martínez 2000).

The following goals guide the structure of my writing:

1) Outline the argumentative structures of degrowth and their relation to global interlocking crises.

2) Identify contemporary feminist discourses which are relevant to the degrowth agenda.

3) Explore how the feminist movement might promote and enhance degrowth, from a transrational approach to peace.

\section{Global interlocking crises}

Economic growth is an intrinsic part of the capitalist global economy. It depends on increasing consumption, production, and resource exploitation and

2. For example, see Perkins 2010; Wichterich 2010; Melchiori 2012; Muraca 2012. The interest in the intersection of feminism and degrowth has increased over the past years, as the Feminism and Degrowth Alliance (FaDA) established in 2016 reflects on one of the main degrowth websites shows (degrowth.info). Further references to publications on degrowth and feminism can be found here. 
is measured by the Gross Domestic Product (GDP). Yet, growth cannot be infinite due to the limits of the resources available to humans, an issue which has been widely acknowledged since the publication of the scientific report The Limits to Growth (Meadows et al. 1972). Consequently, the United Nations called for "development that meets the needs of current generations without compromising the ability of future generations to meet their own needs» (WCED 1987, 1). However, the new international buzzword of sustainability became hijacked by businesses and interpreted as sustainable growth, which defied its purpose as the original problem remained unquestioned (Fournier 2008). Development critiques revealed some of the root problems in the economic development scheme by showing its underlying human and ecological exploitation (Escobar 1994; Esteva 1992; Shiva 1988).

Today, the 6th mass extinction, global warming, habitat destruction, the depletion of resources such as air, water, and soil, the destruction of ecosystems, pollution, and environmental catastrophes represent the evidence of ecological crises fostered or exacerbated by humans (Robbins 2012). The current era is referred to as the Anthropocene, which points to the fact that human influence has shaped global ecological patterns (ibid).

The aforementioned ecological degradation is also linked to social inequality due to the increasingly skewed distribution of finite resources, which has induced struggles over raw materials such as water, oil, and minerals, triggering violent conflict in many cases (McKie 2014). Moreover, detrimental power relations from colonial times continue to exist through resource and cheap labor exploitation (Escobar 1994). As a result, with few exceptions, the gap between rich and poor nations has increased over the past decades, whereby today the richest $1 \%$ is twice as wealthy as the poorest half of the planet (IPCC 2018). This can be termed structural violence (Galtung 1990) since it cannot be ascribed to a concrete perpetrator or group but is related to the structures and uneven power relations. Growth critics have identified crises in the ecological and social realms as linked with the economic system and refer to them as 'global interlocking crises' (Baykan 2007; Brownhill et al. 2012; Trainer 2012; Vail 2011; Kallis 2011; Schneider et al. 2010; Wichterich 2014). The described global situation suggests that there is an urgent need for a profound transformation. With the current economic paradigm that relies fully on economic growth (Oliver-Solà 2010), it is difficult to imagine the required change. Yet, more recent social movements such as the Fridays for Future campaign, that resulted from Greta Thunberg's school strike (Hagedorn et al. 2019), have rekindled a debate around climate 
change and the urgency of political change in Europe. Furthermore, European elections have shown a clear increase of Green and allied parties (9,99\%) as a rising political force (European Parliament 2019). Yet, degrowth remains largely absent from political debate.

\section{Sustainable economic degrowth}

To transform the global unsustainable economic, ecological, and social systems, I argue that the current growth paradigm needs to be questioned and transcended, as the sustainable economic degrowth movement suggests. The degrowth slogan was launched in France in 2001 as décroissance, which translates as 'reduction' (Demaria et al. 2013). During its relatively short life-span, it became a social movement and an activist-led area of studies with increasing importance. Degrowth can be defined as a deliberative, collective process to foster an equitable downscaling of the capacity of production and consumption as well as a reduction of the role of markets and commercial exchanges as a fundamental organizing principle of human lives (Sekulova et al. 2013). Its purpose is to promote human well-being and to enhance ecological condition, at the local and global levels, in the short and long terms.

Degrowth theory has three thematic pillars: the bio-economics pillar, the social pillar, and the pillar of the imaginary. The bio-economics pillar addresses the paradox that the capitalist economic system is based on infinite economic growth, whereby the Earth has finite resources. The exponential economic growth witnessed today is considered as a fundamental characteristic of the modern capitalist economy (Demaria 2013). The continuous production, however, involves the irreversible degradation of a certain proportion of energy and a loss of matter which cannot be reused or recycled (Georgescu-Roegen 1971). Yet, economic accounting mistakes this increase of entropy and the dissipation of resources for wealth creation, so that there is no proper accountability for environmental damage and the depletion of resources.

Degrowth supporters propose re-politicizing the economy in order to uncover it as a self-referential system, an abstract idea which presents itself as an objective reality. It is a call for reframing the meaning of economy. The economic endeavor of shrinking growth is framed in the sense that once the economy has decreased to truly sustainable levels, then living in a steady-state economy or 
zero-growth economy, that is an economy where stocks and flows of energy are constant and at a scale that is sustainable (O'Neill 2011).

The second pillar addresses degrowth's social and political dimensions. While the dominant discourse stemming from economically developed societies asserts that wealth and prosperity derive from innovation and technological progress, encouraging so-called underdeveloped nations to do business and to grow financially, the current agenda entails the introduction of new markets to expand the capitalist logic (Bonaiuti 2012). Development framed in terms of economic growth entails the idea of the so-called trickledown effect, whereby metaphorically speaking a rising tide lifts all boats. It is supposed to help to distribute wealth in a country, whereby those who are worst off in a society automatically benefit if overall wealth in a society increases. However, several studies have shown that this slogan does not hold (Muraca 2012). Instead, generally speaking, in the property-based economy, proprietors have exclusive privileges in contrast to non-proprietors, which creates a capitalist elite and reinforces social inequality (Griethuysen 2009).

Strategies within the degrowth matrix aim to bring the economy back into the hands of people and ecology back into people's responsibility and consciousness. For instance, degrowth scholar Serge Latouche proposes a straight-forward approach synthesized as the 8 R's: to revalue, re-conceptualize, restructure, relocate, redistribute, reduce, reuse, and recycle. These interdependent goals are supposed to provide activist and policy makers with tools for a genuine political program (Latouche 2010) and promote serene, convivial, and sustainable degrowth. The pillar of the imaginary refers to the set of ideas that a society has in common (Bonaiuti 2012). Our imaginary, according to degrowth supporters, is colonized by the idea of growth, which makes imagining a society with merely a shrinking GDP nightmarish, due to consequential skyrocketing unemployment and other social disasters (Latouche 2010). As ecofeminist and postcolonial thinkers among others have recognized, the root cause of the unquestioned growth paradigm can be traced back to the Cartesian mindset in which there is a split between mind and body, reason and matter (Eisenstein 2014). The modern mindset of separation has become one in which the white man seeks to dominate all other species in nature at his disposition. While, in a postmodern era, grand narratives of progress and development have been debunked and deconstructed, the one imaginary that remains globally is that of consumption (Latouche 2010). 
The scrutiny of the economic paradigm has led to totally different perceptions of human interaction from a well-being perspective. For instance, as the Easterlin paradox shows, the increase of consumption per capita shows no correlation with happiness once a certain level of basic needs satisfaction is met (Easterlin et al. 2010). Consequentially, the GDP ought to be abandoned and replaced with more suitable indicators that measure ecological sustainability, social equity and well-being. Moreover, degrowth scholars embraced the notion 'Buddhist Economics' coined by Ernst Schumacher (1973), as it shares some central assumptions with degrowth. Its tenets are as follows:

1. humans are interdependent with nature; hence nature must be central in the economic model;

2. humans are mutually dependent on each other and must also act in ethical ways;

3. the well-being of humans does not mainly depend on material consumption.

Like degrowth, Buddhist Economics does not seek to maximize but to optimize consumption. The ideas of human eco-dependency and interdependency have been taken up by current ecofeminist and feminist ecological economists, activists and scholars in Spain forming the basis of argumentation used by figures such as Yayo Herrero (2010), Alicia Puleo (2008), and Cristina Carrasco (Carrasco and Telló 2013).

While these authors are not explicitly degrowth activists, they do share similar notions which are valuable for the degrowth discourse. As mentioned above, feminist readings of and contributions to degrowth represent a gap that degrowth scholars are aware of (Perkins 2010; Wichterich 2012; Demaria et al. 2013) and needs to be bridged. Hence, the following section inspects feminism(s) in terms of its (their) existing and potential contributions to degrowth.

\section{Contemporary notions of feminism}

The feminist movement today is represented by a range of sub-movements, each exhibiting their own understandings and values. Some scholars argue 
that we are now in the third wave of feminism while others speak about the present in terms of postfeminism (Snyder 2008; Gill and Scharff 2011). The first feminists claiming the third-wave in the 1990s did not strive for overcoming second wave disagreements but instead contained them within a plural vision of feminism with an inclusive and nonjudgmental approach that refrains from controlling the boundaries of feminist politics (Wlodarczyk 2010; hooks 2000). Postfeminism represents an even stronger reification of third-wave principles, whereby in the end, feminism becomes anything that someone who is a selfproclaimed feminist chooses it to be and this is nobody else's business (Snyder 2008; Gill and Scharff 2011). Both concepts contrast with the second wave from the 1970s, since they describe a moment of plural individualistic discourses that stress individuality and reinforce consumer identity without paying critical attention to larger structures, thereby forming a neo-liberal market of ideas (Budgeon 2011).

In light of the above, when considering contemporary feminism, it is crucial to note it does not stand in opposition to the economic growth paradigm. To the contrary, its fragmentary character makes it perfectly blend into unquestioned consumerism and individualism of the economic growth paradigm. Hence, in the search for an ally to enhance and promote the degrowth movement, feminism in general is not suitable. The question of what can be considered feminist needs to be discussed. In this context, I promote a version of feminism which is a reflection of the characteristics which feminism would need not to contradicting itself while guarding the spirit of a positive transformation of society.

This feminism can be summarized in four points:

1. It pursues gender equality, recognizing that there is such a thing as patriarchy and that feminism is a movement to abolish sexism (hooks 2000). Patriarchy is a system of oppression whereby the feminine is seen as inferior to the masculine and that people of different genders suffer in distinct ways (Stanley and Wise 2002).

2. It recognizes the underlying cultural roots of patriarchal violence. Therefore, formal equality of women, in terms of women's quota in decision-making positions and overcoming the wage-gap, is not sufficient to change the system which is dominated by male-centered values. 
3. It considers that domination stretches into other categories such as class, race, sexuality, age, or religion, among others. In other words, feminism necessitates intersectional thinking (Crenshaw 1990).

4. The feminism I endorse recognizes the parallels between the oppression of women and the domination of nature. This aspect turns the feminism I endorse into an eco-feminism (Puleo 2008).

\section{Feminist contributions to degrowth}

While the contributions that specifically address degrowth from a feminist perspective are limited to date, scholars from several related fields provide reflections that are enriching for degrowth (see Waring 1988; Kaur 2012; Tong 2009). In the following, I shortly sketch three kinds of interrelated feminist contributions to degrowth which I refer to as care, sharing, and subsistence perspectives.

The care perspective refers to the idea that so-called women's work in the care and reproductive sector, and nature are fundamental and irreplaceable foundations of the economy (Perkins 2010). These unpaid services and goods dwarf the official economy in value, yet they are usually not considered important enough for policy deliberations (Pietilä 1997; d'Alisa, Deriu and Demaria 2015). While reproductive work has been moving into the formal sector, thereby gaining visibility, this has led to a further entrenchment of the capitalistic logic. Hence, rather than revaluing reproductive labor, this process subjugates many women to market principles, resulting in higher profits for capitalists. Additionally, the feminization of women's work has not increased equity in terms of work distribution. As it seeks to de-commodify labor, degrowth thus faces the challenge of addressing relative values, undervaluation, and justice related to exploitation of underpaid female workers, and of nature.

Contributions focusing on sharing benefits and burdens argue for justice and community-building as well as de-alienation. In this context, Perkins vision of the commons (2010) widely refers to people working cooperatively, to build methods of production, service provision, and exchange that create value and well-being as they integrate ecological care, justice, and long-term planning by employing their communities' abilities at their best. The subsistence perspective is based on an awareness that the degradation and oppression of women, the exploitation of 
labor, of nature, and of peoples in the Global South are the prerequisites for the growth paradigm (Mies and Bennholdt-Thomsen 2000). It suggests to abandon all practices related to consumerist and productivist lifestyles, embracing much simpler paths that reconnect with nature and by alternative means. Its driving principle is that everything that produces life has to become primary and earning money secondary and peripheral again. To foster alliances among both movements, a direct mutual engagement and interdisciplinary dialogue among growth-critical feminisms and degrowth would be necessary. The following section deals with the ways in which a transrational peace perspective envisions such an alliance.

\section{Transrational feminist degrowth}

The transrational vision in peace studies is proposed by Wolfgang Dietrich (2018), which is derived from John Paul Lederach's pyramid of conflict work (2004), adding a third dimension and several layers to the diagram. Lederach seeks to overcome gaps between theory and practice and to promote a paradigm of conflict transformation with a long-term engagement rather than just mitigating episodes of violence (ibid.). This resonates well with degrowth as an activist-led science that promotes long term change of social structures. Dietrich introduces four peace families, which present different viewpoints from which to analyze peace and conflict-related issues: the energetic, the moral, the modern, and postmodern peace family. These understand peace as harmony, as justice, as security as truth, respectively, and can be allocated to interior, exterior individual and collective aspects. In essence, the transrational perspective strives for a broader systemic understanding of conflict as a relational phenomenon. Since providing a thorough analysis of degrowth from a transrational perspective exceeds the scope of this article, I focus merely on providing a reflection on how informing my feminist perspective with a transrational approach to peace work can enhance degrowth due to its systemic and holistic nature. In the light of this, I suggest a transcendence of the epistemological narrowness of modern rational thinking that is still present in both degrowth philosophy and feminist thinking at large. This tendency visible in degrowth presents itself as a matrix of alternatives with a plurality of ideas on how to abandon growth rather than embrace something else (Schneider et al. 2010; Brownhill et al. 2012). Despite 
the fact that feminist research methods have transcended much of the positivist approaches of mainstream science (Stanley and Wise 2002), the poststructural necessity to avoid essentialism seems to limit more holistic understandings of feminist issues in relation to other oppressive structures such as the growth paradigm, particularly when it comes to action (Gaard 2011). In this context, I suggest a shift whereby interpretation and meaning-making gains prominence over the identification of a scientific, objective truth. This approach has allowed me to integrate insights that transcend feminist literature, while dealing with the problems of growth-based economy and patriarchy.

Concretely, I draw from economist Bernard Litaer's inspirational work Money Mystery (2002), which has provided an explanation of the imbalances that revolve around money by considering Taoist philosophy as well as Jungian psychology and archetypes. Litaer points to a correlation of the neglect of what can be called the feminine principle or, in Taoist terms, the Yin principle, and the rise of the dominant economic system based on growth, greed, and competition, which refer to yang qualities. The tendency of dominating Yang qualities prevails in both, the economic order and in terms of patriarchal culture.

Moreover, Litaer exposes that the Great Mother archetype has been buried into the unconscious for centuries (ibid). The presence of a divine feminine in the imaginary of the human species is evident in thousands of cultural artifacts that have been present for centuries and later disappeared and repressed. Beyond being a symbol of femininity, the Great Mother archetype represents abundance and the principles of money, sex, and death, which are taboo topics in Western society (Dibella 1980). In general terms, archetypes manifest their shadows when they are repressed (Jung 2014). The Great Mother archetype manifests the shadows of greed and fear of scarcity. The assumption of scarcity is the basis of the economic system as we know it and regarded as normal within economics and greed certainly is a symptom of glut and economic growth mentality.

From a transrational approach, Litaer's work represents a systemic interpretation of oppression that shows the connection between two different paradigms: the economic and the patriarchal system. What is striking about his interpretation is that he addresses it at the level of symbolism and cultural roots of our imaginary. Hence, one can argue that Litaer deals with the inner collective quadrant of the related conflicts of patriarchy and economic growth. 


\section{Conclusions and further research}

A feminism that can enhance and promote degrowth in fruitful ways ought to be critical of the growth paradigm and aware of the link between ecological and gender oppression. While I have identified three sets of ideas by which feminism contributes to degrowth -sharing, care, and subsistence perspectives- an active dialogue between feminist and degrowth perspectives is necessary.

Moreover, both degrowth and feminism show a certain epistemological narrowness in which the pluralist and approaches of both lack mutual interpellation, which evades more holistic, perhaps bolder understandings of their interrelatedness. A transrational approach to peacebuilding might serve to bridge this gap and approach feminism and degrowth from a relational, systemic perspective. Transdisciplinary literature such as that provided by Bernard Litaer can help to elucidate the foundations of an imaginary which has fostered both, patriarchal, and growth-based societies. His interpretations are useful to enhance the capacity to gain meaning as opposed to the search for objective scientific truth, in the realms of degrowth, feminism, and a normative lens of peace studies. Further research includes identifying practical examples of the re-emergence of the feminine principle and testing in what way the theories presented can be put into dialogue with one another through practice.

\section{References}

Baykan, Baris Gencer. 2007. «From limits to growth to degrowth within French green politics». Environmental Politics 16 (3): 513-517.

Bock, Marisol C. 2015. «Considering sustainable degrowth from a feminist transrational perspective». MA dissertation, Universitat Jaume I. http://repositori. uji.es/xmlui/handle/10234/145488.

Bonaiuti, Mauro. 2012. «Degrowth: Tools for a complex analysis of the multidimensional crisis». Capitalism Nature Socialism 23 (1): 30-50.

Brownhill, Leigh, Terisa E. Turner, and Wahu Kaara. 2012. «Degrowth? How about some 'de-alienation'?» Capitalism Nature Socialism 23 (1): 93-104.

Budgeon, Shelley. 2011. «The contradictions of successful femininity: Third-wave feminism, postfeminism and 'new' femininities». In New Femininities, edited 
by Rosalind Gill and Christina Scharff, 279-292. London: Palgrave Macmillan.

Carrasco, Cristina and Enrique Telló. 2013. «Apuntes para una vida sostenible». Tejiendo alianzas para una vida sostenible. Consumo crítico, feminismo y soberanía alimentaria, 11-14, Barcelona: Xarxa de Consum Solidari y Marcha Mundial de las Mujeres.

Crenshaw, Kimberley. 1990. «Mapping the margins: Intersectionality, identity politics, and violence against women of color». Stanford Law Review 43 (6): 1241.

D’Alisa, Giacomo, Marco Deriu, and Federico Demaria. 2015. «Care»In

Degrowth. Vocabulary for a New Era, edited by Giacomo D'Alisa, Federico Demaria, and Giorgios Kallis, 91-94. New York: Routledge.

Demaria, Federico, Francois Schneider, Filka Sekulova, and Joan Martínez-Alier. 2013. "What is degrowth? From an activist slogan to a social movement». Environmental Values 22 (2): 191-215.

Dibella, Geoffrey. 1980. «Mastering money issues that complicate treatment: The last taboo». American Journal of Psychotherapy 34 (4): 510-522.

Dietrich, Wolfgang. 2018. Elicitive Conflict Mapping. London: Palgrave Macmillan UK.

Easterlin, Richard, Laura McVey, Malgorzata Switek, Onnicha Sawangfa, and Jacqueline Zweig. 2010. «The happiness-income paradox revisited». Proceedings of the National Academy of Sciences of the United States of America 107 (52): 22463-22468.

Eisenstein, Charles. 2014. Sacred Economics: Money, Gift, and Society in the Age of Transition. Kindle Edition, New York: Evolver Editions.

Escobar, Arturo. 1994. Encountering Development, Princeton: Princeton University Press.

Esteva, Gustavo. 1992. «Development». In The Development Dictionary: A Guide to Knowledge as Power, edited by Wolfgang Sachs, 6-25. London: Zed Books Ltd.

European Parliament. 2019. «European elections results». European Parliament. http://www.europarl.europa.eu/news/en/press-room/elections-press-kit/8/ european-elections-results.

Fournier, Valerie. 2008. «Escaping from the economy: The politics of degrowth». International Journal of Sociology and Social Policy 28 (11/12): 528-545. 
Gaard, Greta. 2011. «Ecofeminism revisited: Rejecting essentialism and re-placing species in a material feminist environmentalism». Feminist Formations 23 (2): 26-53.

Galtung, Johan. 1990. «Cultural Violence». Journal of Peace Research 27 (3): 291305.

Georgescu-Roegen, Nicholas. 1971. «The entropy law and the economic process». The Economic Journal 83 (330): 476.

Gill, Rosalind, and Christina Scharff, eds. 2011. New Femininities. London: Palgrave Macmillan.

Griethuysen, Pascale van. 2009. «Why are we growth-addicted? The hard way towards degrowth in the revolutionary western development path». Journal of Cleaner Production 18 (6): 590-595

Hagedorn, Gregor, Peter Kalmus, Michael Mann, Sara Vicca, Joke Van den Berge, Jean Pascal Van Ypersele, Dominique Bourg, et al. 2019. «Concerns of young protesters are justified». Science 364: 139-140.

Herrero, Yayo. 2010. «Decrecimiento y mujeres. Cuidar: Una práctica política anticapitalista y antipatriarcal». In Decrecimientos, edited by Carlos Taibo, 17-30. Madrid: Los Libros de la Catarata.

hooks, bell. 2000. Feminism is for Everybody: Passionate Politics. Cambridge: South End Press.

IPCC (Intergovernmental Panel on Climate Change). 2018. Special Report: Global Warming of $1.5^{\circ} \mathrm{C}$. IPCC. Accessed June 20, 2019. https://wir2018.wid.world/

Jung, Carl G. 2014. The Archetypes and the Collective Unconscious. New York: Routledge

Kallis, Giorgios. 2011. «In defence of degrowth». Ecological Economics 70 (5): 873880. doi: https://doi.org/10.1016/j.ecolecon.2010.12.007

Kaur, Gurreet. 2012. "An exegesis of postcolonial ecofeminism in contemporary literature». International Journal of Law and Social Sciences 2 (1): 190-195.

Latouche, Serge. 2010. «Degrowth». Journal of Cleaner Production 18: 519-522.

Lederach, John Paul. 2004. The Moral Imagination. New York: Oxford University Press.

Litaer, Bernard. 2002. The Mystery of Money. Munich: Riemann Verlag.

Martínez Guzmán, Vicent. 2000. «Saber hacer las paces. Epistemología de los estudios para la paz». Convergencia 23: 49-96.

McKie, Robin. 2014. «Earth faces sixth 'Great Extinction' with $41 \%$ of amphibians set to go the way of the dodo». The Guardian. Accessed June 20, 2019. https:// 
www.theguardian.com/environment/2014/dec/14/earth-faces-sixth-great-extinction-with-41-of-amphibians-set-to-go-the-way-of-the-dodo

Meadows, Donella H., Dennis L. Meadows, Jorgen Randers, and William W. Behrens III. 1972. The Limits to Growth. New York: Universe.

Melchiori, Paola. 2012. «Feminism and degrowth: Parallelisms and intersections. The contribution of feminism to the economy of degrowth». Degrowth Conference Venice 2012 (January): 1-12.

Mies, Maria, and Veronika Bennholdt-Thomsen. 2000. The Subsistence Perspective. New York: Zed Books.

Millett, Kate. 2014. Sexual Politics. New York: Columbia University Press.

Muraca, Barbara. 2012. «Towards a fair degrowth-society: Justice and the right to a "good life" beyond growth». Futures 44 (6): 21-31.

Oliver-Solà, Jordi. 2010. «Prosperity without growth? - The transition to a sustainable economy». Journal of Cleaner Production 18 (6): 596-597.

O'Neill, Daniel W. 2011. «Measuring progress in the degrowth transition to a steady state economy». Ecological Economics. doi: http://doi.org/10.1016/j. ecolecon.2011.05.020.

Perkins, Patricia E. 2010. «Equitable, ecological degrowth: Feminist contributions». In 2nd Conference on Economic Degrowth. Barcelona, 1-8.

Pietilä, Hilkka. 1997. «The triangle of the human economy: Household-cultivation-industrial production. An attempt at making visible the human economic in toto». Ecological Economics 2: 113-128.

Puleo, Alicia. 2008. «Libertad, igualdad, sostenibilidad. Por un ecofeminismo ilustrado». Isegoría 38: 39-59.

Rich, Adrienne. 1985. «Notes toward politics of location». In Women, Feminist Identity and Society in the 1980s, edited by Myriam Díaz-Diocaretz and Iris M. Zavala, 7-22. Amsterdam: John Benjamins.

Robbins, Paul. 2012. Political Ecology: A Critical Introduction. 2nd edition. Malden: Wiley-Blackwell.

Schneider, Francois, Giorgios Kallis, and Joan Martínez-Alier. 2010. «Crisis or opportunity? Economic degrowth for social equity and ecological sustainability. Introduction to this special issue». Journal of Cleaner Production 18 (6): 511518.

Schumacher, Ernst Friedrich. 1973. Small is Beautiful: Economics as if People Mattered. New York: Harper Perennial. 
Sekulova, Filka, Giorgos Kallis, Beatriz Rodríguez-Labajos, and Francois Schneider. 2013. «Degrowth: From theory to practice». Journal of Cleaner Production 38: 1-6.

Shiva, Vandana. 1988. Staying Alive. Women, Ecology and Survival in India. London: Zed Books Ltd

Snyder, R. Claire. 2008. "What is Third Wave Feminism? A New Directions Essay.» Signs 34 (1): 175-196.

Stanley, Liz, and Sue Wise. 2002. Breaking Out Again. Hoboken: Taylor and Francis.

Tong, Rosemarie. 2009. Feminist Thought. A More Comprehensive Introduction. 3rd edition. Boulder: Westview Press.

Trainer, Ted. 2012. «De-growth: Do you realise what it means?» Futures 44 (6): 76-85.

Vail, John. 2011. «Decommodification and egalitarian political economy». Politics and Society 38: 310.

Waring, Marilyn. 1988. If Women Counted: A New Feminist Economics. San Francisco: Harper \& Row.

Wichterich, Christa. 2012. «The future we want. A feminist perspective». Heinrich Böll Stiftung 21: 1-50.

Wichterich, Christa. 2014. «Searching for socio-ecological and socio-economic transformation - a feminist perspective on the 4th De-growth Conference in Leipzig». In: 4th De-growth Conference in Leipzig. Leipzig.

Wlodarczyk, Justyna. 2010. Ungrateful Daughters: Third Wave Feminist Writings. Cambridge: Cambridge Scholars Publishing.

WCED (World Commission on Environment and Development). 1987. Our Common Future. Oxford: Oxford University Press. 


\title{
The focus on interculturality: A quantitative and qualitative bibliometric study on legal interpreting research
}

\author{
Ana Vázquez Fernández \\ anavazquezfdez@gmail.com
}

Bibliometrics started as a fundamentally quantitative approach which has now begun to integrate qualitative aspects. In this chapter I present a mixedmethods approach which brings together bibliometrics and Philip Mayring's qualitative content analysis. With this methodology I tackle the issue of how interculturality has been incorporated into research on legal interpreting, positing that, in accordance with the cultural shift that has taken place in Translation and Interpreting Studies, the latest studies will incorporate the intercultural dimension in new and more diverse ways. After building a corpus made up of articles on legal interpreting, I applied Mayring's summarising content analysis and inductive category formation. I identified twenty different ways in which interculturality is brought into research on legal interpreting. It appears in relation to different sub-topics of sorts, such as the issue of cross-cultural equivalence, among others, as well as in different parts of the research (as part of the research question or the theoretical considerations, for instance). Close analysis of the results showed that the main issue related to this topic seems to be that of the interpreter's role: mere conduit or active broker? The results also show that further clarification is needed on what is culture and what are cross-cultural issues. Finally, while the initial hypothesis was only partially confirmed, the insights gained further prove 
the usefulness and potential of the mixed-methods approach presented in this chapter.

Keywords: interculturality, culture, translation and interpreting studies, legal interpreting, bibliometrics, qualitative content analysis

\section{Introduction}

Bibliometrics and scientometrics, which nowadays have become almost synonymous (Glänzel 2003, 6; Grbić and Pöllabauer 2008, 297), refer to the scientific description and evaluation of scientific production. They not only describe but also evaluate the science being conducted. Bibliometrics is widely used nowadays to measure the productivity and impact of journals and researchers throughout all fields (Geisler 2000, 154, 157; Bellis 2015, 23). One of its biggest contributions has been to enable us to gather information about the evolution, dynamics, and trends within different academic disciplines. What started as a fundamentally quantitative approach has now begun to integrate qualitative methods coming from fields such as sociology or text linguistics (Grbić and Pöllabauer 2008, 298; Martínez-Gómez 2015, 207). This chapter presents an approach to incorporate this qualitative dimension to bibliometric studies by including methods from communication analysis. I have already successfully applied this methodology to the study of the gender dimension in Interpreting Studies (Vázquez Fernández 2019) and, in this chapter, I aim to further prove its usefulness and potential in other fields by exploring the study of interculturality within legal interpreting.

\section{Interculturality in legal interpreting and translation}

Culture, and therefore, interculturality, have been notoriously difficult to define (Katan 2018, 25). As the American anthropologist and cross-cultural researcher Edward Hall put it, «culture is not one thing, but many» $(1973,217)$. 
He distinguished three facets in culture (ibid., 217-218): technical culture, or culture as the arts and other manifestations of human intellectual achievement; formal culture, or culture as learned appropriate behaviour, what should and should not be done; and informal culture, or culture as unconscious mental processes that influence judgements, feelings or behaviour (Katan 2018, 25-28). Culture in all of its facets plays a central role in communication. Hall actually identified them with each other: "culture is communication and communication is culture» $(1973,217)$. In the next paragraphs I will review the relationship between culture and a specific form of communication: legal interpreting and translation. All of this will serve as the required (see section 3.2) theoretical foundations for the analysis that I will carry out in this paper.

\section{Legal interpreting and translation and culture}

Legal interpreting and translation have a long, recognised relationship with the evolution of cultures and cross-cultural contacts: «the first legal translation is said to be a bilingual peace treaty signed between the Egyptians and the Hittites in 1271 BCE, which places LIT ahead of other specialisms» (Monzó-Nebot 2018, 464). However, the traditional views that have dominated legal interpreting and translation (and translation and interpreting in general) have represented the translator/interpreter as a conduit whose role is limited to the mere transfer of words (Cooke 1995), and translation as simply a matter of contrastive linguistics. This has changed over the course of the four past decades, in which a 'cultural shift' has taken place within Translation and Interpreting Studies (Harding and Carbonell Cortés 2018, 1). Regarding translation and interpreting in legal settings, this cultural shift challenging the view of the translator/interpreter as a machine-like being has taken place in the literature (Vidal Claramonte 2013) and before the courts, although this is a recent development (MACI 2016; Kracum 2014).

Traditionally, legal interpreting and translation have been expected to limit their scope to mere linguistic transfer, without tackling cultural issues. And yet the law and the language used to express the law were born and evolved within a specific culture, thus becoming deeply embedded in it. Due to the performative character of the law, form is identified with function: the language of the law is the law. When this is taken into consideration, the challenge of translating 
culturally rooted terms might seem daunting, and may even lead to the issue of untranslatability: how do we take a legal text whose foundations are culturally specific, and make it work in a new system?

\section{The issue of cross-cultural equivalence}

Cross-cultural 'equivalence' has been thoroughly discussed, and the seemingly most satisfying answers are the concepts of legal equivalence and functional equivalence. The former compares which terms or discursive genres are used for a certain notion or legal situation across legal systems. This is only possible when the same notion exists in different legal systems, which is usually the case either in bilingual legal systems (like Canada) or in legal systems with a shared tradition, i.e. within the same legal family (Monzó-Nebot 2018, 467). The functional approach, on the other hand, looks into how different cultures solve particular legal problems and searches for strategies to find formulations in the target language that convey «the part of the source term's legal meaning that is conducive to the target text's purpose» (ibid., 468).

Another notion that might answer the issue of cross-cultural 'equivalence', while raising other questions regarding the interaction of the law and culture in an increasingly transcultural world, is that of intercultural legal discourse. The philosopher Otfried Höffe succinctly defines it like this: «the basic idea of the intercultural discourse can be formulated as follows: Whatever we consider to be fundamentally important, we can expect to discover in different cultures, too» $(2015,362)$. Höffe's proposal posits the existence of a universal legal culture of sorts. This might be a bridge to enable 'translatability' between different legal systems. The fundamental elements that we consider to be basic justified claims can be found in other cultures; the same goes for whatever we might consider basic blameworthy actions or behaviours. There seems to be a fundamental, cross-cultural concept of good/justice, as well as one of wrongdoing/injustice. Intercultural discourse demands respect for each culture's distinctiveness and avoids the dangers of ethnocentrism. Finally, Höffe distinguishes different levels at which the intercultural legal discourse can take place $(2015,362)$ : 
- Within the history of moral and legal thought, by trying to determine the origin of morality and law characteristic of different cultures, and the various cross-cultural influences.

- Within the comparison of different legal cultures, by focusing on the shared moral legacy of humankind.

- Within the justification of moral and legal principles, by using premises that are universally valid because they are founded on human reason and anthropological elements, while also employing a distinction between universality and uniformity, thus remaining open to the distinctiveness of different cultures.

- Within moral and legal practice, by demanding that the principles guiding said practices be realised with caution, leaving space for each culture to go through learning processes regarding human rights and international law.

In this paper, I will present a case study focused on interculturality in legal interpreting research. My aim is to see to what degree interculturality has been integrated into research on legal interpreting. To that end, I will analyse a corpus of scientific publications on the topic using the methodology that I will present in the following section. I will posit that, in accordance with the cultural shift that has taken place within Translation and Interpreting Studies, and in legal interpreting and translation more specifically, the most recent investigations will tackle interculturality in new and more diverse ways.

\section{Integrating quantitative and qualitative approaches}

In this chapter, I aim to present a mixed-methods approach for the analysis of interculturality in legal interpreting. This approach integrates quantitative and qualitative elements by combining bibliometrics with Philipp Mayring's qualitative content analysis.

\section{Bibliometrics}

Since the end of World War II, bibliometrics has become synonymous with the scientific description and evaluation of scientific production. In the 1950s, 
the American chemist Eugene Garfield began to evaluate papers systematically based on the literature used and cited. This evaluating activity has grown into a vast collection of data, with comprehensive databases recording all the information needed for the majority of bibliometric analysis (Ball 2018, 1-2). These databases «include citations or bibliographic references, and sometimes abstracts of literature» (Chowdhury 2010, 17). Although they present many advantages, they also have weaknesses, most notably in relation to certain biases that they might present, such as an inclination «towards 'popular' fields, longestablished publications, and English-speaking works, among others» (MartínezGómez 2015, 209). In spite of these limitations, bibliographic databases remain the main source of data for bibliometric analyses.

Bibliometric analysis can be used to examine the evolution, trends, and dynamics of a discipline. Translation and Interpreting Studies have successfully used this approach to analyse its development. Some of the pioneers in this field are Gile, Grbić, Pöllabauer or Pöchhacker. The former actually used this method to claim for Interpreting Studies to be considered as a field of its own. In Translation Studies, bibliometric analyses have been carried out in the fields of scientific (Franco Aixelá 2004), medical (Franco Aixelá 2010), and audiovisual translation (Franco Aixelá and Orero Clavero 2005). With regards to interpreting, some of the modalities studied with this methodology have been conference interpreting (Gile 2000), interpreting in police settings (Pöllabauer 2006) and in public services (Grbić and Pöllabauer 2008), among others. Current trends in Translation and Interpreting Studies have also been identified through a bibliometric lens (Shlesinger 2009; Yan et al. 2013). Finally, this approach has also proven fruitful in order to characterise new sub-fields of study such as nonprofessional translation and interpreting (Martínez-Gómez 2015).

Parallel to all of these studies, bibliographic databases specific to Translation and Interpreting Studies have also been established. We have identified databases such as E-lectra (Monzó-Nebot 2010), specific to legal interpreting and translation, BITRA (Franco Aixelá 2001-2019), or Translation Studies Bibliography (Gambier and van Doorslaer 2004b-2019). BITRA belongs to the University of Alicante and has been assembled and coordinated by Javier Franco Aixelá. The latter belongs to John Benjamins, whose series Benjamins Translation Library is «the leading and most extensive book series in translation studies» (Pöchhacker 2011, 7). The Translation Studies Bibliography is edited by Yves Gambier and Luc van Doorslaer and aims to capture a broad scope of publications related to Translation and Interpreting Studies. To that end it uses 
a working definition of Translation and Interpreting Studies as a «broad field of transfer and mediation, containing aspects of intra- and interlingual translation, adaptation, interpreting, reformulation/rewording, localisation/multimedia translation, language mediation and terminology/documentation» (Gambier and van Doorslaer 2004-2019a). Gambier and van Doorslaer also acknowledge the interdisciplinary nature of Translation and Interpreting Studies, thus taking into account publications from other disciplines which might be relevant and of interest to the field. Due to its interdisciplinary nature and the different types of publications included, as well as its accessibility through the University Jaume I, I have chosen this database in order to conduct the searches for my case study.

\section{Qualitative content analysis}

Qualitative Inhaltsanalyse, or qualitative content analysis in English, is an approach developed by the German sociologist, psychologist, and educator Philipp Mayring with the aim of integrating a qualitative dimension to the already existing methodology of content analysis. This had been developed during the 20th century within the field of communication sciences as a technique for «the systematic analysis of large quantities of textual data from the growing mass media» (Mayring 2004, 266). Qualitative content analysis enables the systematic examination of large textual quantities (textual corpus) through different procedures: summarising content analysis, inductive category formation, explicating content analysis and structuring content analysis (ibid., 268-269; see also Mayring 2016, 115). These analytical procedures are based on the following fundamentals: understanding of the material to be analysed as embedded in its context of communication, systematic nature of the analysis (based on rules, theory-dependency, gradual procedure), measurability against quality criteria, inter-coder reliability, and incorporation of quantitative analytical procedures in a justified way (Mayring 2004, 267).

The different procedures of Mayring's qualitative content analysis serve different goals. Summarising content analysis and inductive category formation are the best suited for the aim of this paper. The former will allow me to reduce the texts in my corpus «in such a way that the essential contents are preserved, but a manageable short text is produced» (ibid., 268). The latter enables the 
gradual development of categories, which in turns offers an overview of the different ways in which interculturality is approached in the corpus.

\section{Summarising content analysis}

Summarising content analysis is «always suitable if one is only interested in the content level of the material and is required to condense the material into a manageable short text» (Mayring 2004, 268). It can be done through a series of techniques based on the psychology of text processing: omission, generalisation, construction, integration, selection and bundling (ibid.). Before carrying out the analysis, the desired level of abstraction is defined. In this case, I am interested in all propositions dealing with interculturality. The next is applying the aforementioned techniques: first, selection (already general and summarising propositions are kept without changes), generalisation (propositions implicit in others are replaced by the more abstract, general proposition), and omission (repeated propositions with the same meaning are omitted); and afterwards, bundling (content-related propositions distributed throughout the whole text are bundled in one), construction (a global proposition is formulated based on more specific others), and integration (propositions that can be integrated into more general ones are omitted) (Mayring 2016, 96). If the end result does not match the desired level of abstraction, the procedure can be repeated once more.

\section{Inductive category formation}

Summarising content analysis can be completed with inductive category formation. In this step, the procedures of the first are used to develop categories gradually from some material. In this paper, I will be developing categories from the summarised text resulting from the preceding step (Mayring 2004, 268). Before doing so, we must also determine the level of abstraction. In this case, I am interested in building categories that correspond to different aspects of interculturality. Mayring's inductive category formation also calls for determining, prior to the actual analysis, which characteristics a category might 
have. This is done based on the subject matter, research question, and relevant theoretical considerations.

In this case study, a category might be built based on any text passage that relates to interculturality. A priori after examining the research question and the theoretical considerations presented, I expect to build categories that relate to the following aspects: different definitions of culture, different perceptions of the interpreter's role, the relation between law, language and culture, the issue of cross-cultural equivalence, intercultural (legal) discourse, interculturality as part of the research question in each article, and interculturality as part of the theoretical considerations in each publication.

The next step is going through each line of the material. Once a passage is found which fits the definition of a category, a label is assigned. The next time that we come across a suitable passage, we either subsume it within the same existing category or create a new one. After going through between $10 \%$ and 50 $\%$ of the text, the established categories are reviewed. Necessary adjustments are done and then we may analyse the whole text. If the end result does not match the established abstraction level and the category definitions are not clear, then the whole analysis needs to be started again.

\section{The study}

\section{The data}

The data for this study are scientific publications within the sub-field of legal interpreting which integrate interculturality. As already stated, I selected John Benjamin's Translation Studies Bibliography as my bibliographic database. For my analysis, however, I needed the full texts. The language chosen was English, which means I will only examine how interculturality is tackled in English-language research on legal interpreting, since bringing in publications in other languages would also bring in different conceptions of culture and interculturality. For my searches I used the combined keywords 'interculturality $=$ transculturality $=$ cros s-culturality' and 'legal interpreting', as well as 'interculturality=transculturali ty $=$ cross-culturality' and 'court interpreting.' The first selected keyword serves to capture any publications dealing with issues related to interculturality, which 
might also appear under the terms transculturality and cross-culturality in the searched database. The keywords 'legal interpreting' and 'court interpreting' were used to capture any item dedicated to interpreting in a legal setting, not just in a trial context.

The first query, using 'legal interpreting', yielded six results, while the second one hit only five. Three titles were overlapping, so I ended up with a total of eight different publications. Of those, only five were either open access or accessible through the Berlin State Library or the University Jaume I. They were the following (listed in chronological order from newest to oldest):

- Hale, Sandra. 2014. «Interpreting Culture. Dealing with Cross-Cultural Issues in Court Interpreting».

- Barsky, Robert F. 1996. «The Interpreter as Intercultural Agent in Convention Refugee Hearings».

- Cooke, Michael S. 1995. «Understood by All Concerned? Anglo/Aboriginal Legal Translation».

- Dunnigan, Timothy, and Bruce T. Downing. 1995. «Legal Interpreting on Trial: A Case Study».

- Hammond, Matt. 1995. «A New Wind of Quality from Europe: Implications of the Court Case Cited by Holz-Mänttäri for the U.S. Translation Industry».

\section{Summarising content analysis}

The aim behind the summarising content analysis procedure was to reduce the material to a shorter text which was still a faithful reflection of how the original tackled interculturality. The starting corpus was composed of five articles (around 80 pages). After applying the first techniques of summarising content analysis, namely selection, generalisation, and omission, the corpus was reduced to nine pages or 116 propositions. The next step was using the techniques of grouping, construction, and integration. In the end, the original corpus was reduced to five pages or 82 propositions that were still a faithful reflection of interculturality in the original texts. The following graphic depicts the distribution of those 82 propositions per article in the corpus: 


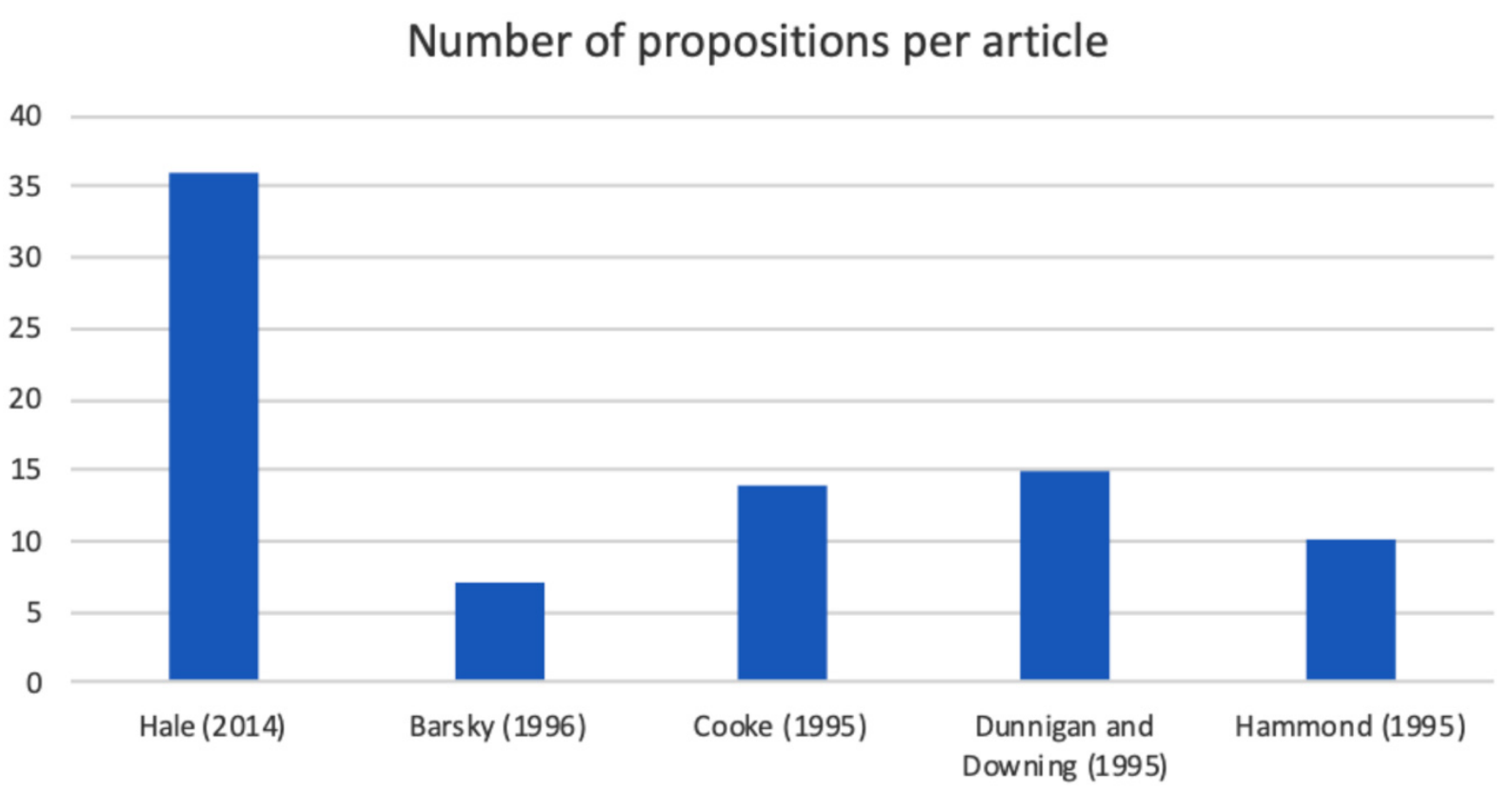

number of propositions per article

Figure 1. Number of propositions per article

As can be seen, most of the propositions regarding interculturality can be found in Hale's article. I will discuss the significance of these results later on.

\section{Inductive category formation}

In this step, I built categories based on the analysed text while bearing in mind the research question, research object, and relevant theoretical considerations. Twenty categories were built on the basis of the theoretical foundations laid, reflecting different ways in which interculturality is brought into research in legal interpreting. As expected, categories pertained to different definitions of culture, different perceptions of the interpreter's role, the relation between law, language and culture, the issue of cross-cultural equivalence, intercultural (legal) discourse, interculturality as part of the research question in each article, and interculturality as part of the theoretical considerations in each publication. In addition to those categories, the analysis revealed other aspects in which interculturality played a role, such as interculturality as an argument in court, who is responsible for managing 
interculturality, interculturalityas an element for the professionalisation of translators/ interpreters, and the issue of how one becomes qualified in interculturality. Another key issue detected was the question of how to define culture and/or interculturality. Only two articles, Hale's and Cooke's, include interculturality as their research object and, therefore, use it as well in their theoretical framework. By far, the biggest aspect related to interculturality that is tackled in the corpus is that of whether or not the interpreter should act as a cultural mediator or broker of sorts.

As for the distribution of the categories, fourteen different categories have been found in Hale's article, seven in Barsky's, ten both in Cooke's and Dunnigan and Downing, and only five in Hammond's. Hale's investigation presents the highest variety in aspects of interculturality among those taken into consideration. Each article tackles an average of nine different issues related to interculturality.

\section{Discussion}

In this section, I will present the results of my study. First, I will analyse how each article in the corpus tackles interculturality. Then I will showcase the common, most salient elements detected, as well as the limitations of this study. Finally, in light of the results, I will confirm or reject the claim previously posited: the latest articles in legal interpreting, in accordance with the cultural shift that has taken place in Translation and Interpreting Studies, and in legal interpreting and translation, will take interculturality into account in new and more diverse ways.

After applying Mayring's content summarising analysis I obtained 82 propositions which reflect how the articles in the corpus take into account interculturality. By far, Hale's 2014 study is the one that dedicated more lines to this issue. This is in accordance with her research object since her aim was to find out the expectations of both judicial officers/tribunal members and interpreters regarding cross-cultural misunderstandings. Logically, she also presents different theoretical considerations related to this issue. The inductive category formation revealed the different ways in which Hale considers interculturality. Her paper focuses mainly on how interculturality relates to the interpreter's role: should s/he be allowed to act as a cultural broker, or should his/her role be limited to that of linguistic transfer? One of the main points made by Hale regards the lack of clarification on what exactly are 'culture' and 'cross-cultural differences'. 
She presents conceptualisations of culture such as culture as appropriate learned behaviour, culture as a series of unconscious mental processes and culture as a dynamic being. Hale also touches on how translator's/interpreter's codes of ethics reflect (or lack reflection) on cross-cultural differences and how to act when they arise. She also mentions the issue of qualifications, that is, which skills or knowledge are required to become an intercultural expert?

As for Barsky, his investigation also focuses mainly on the interpreter's role in the face of cross-cultural issues: should s/he be a fully-fledged intercultural agent or just a conduit? He recognises the cultural nature of the law and the consequences deriving from it. Barsky also presents culture as a series of unconscious mental processes linked to the spirit of a particular people or nation. Cooke's research has interculturality in legal settings as his subject matter and, therefore, as part of his theoretical considerations. Cooke refers to the cultural nature of both the law and its language, and he reflects on the issue of crosscultural equivalence. He presents culture as something shared and, like Barsky, relates to a certain people. His article is mostly focused on legal translation, but he reminds us throughout it that the same applies in legal interpreting.

Dunnigan and Downing's text brings forth a new dimension for interculturality: as an argument to be used in court. In their review of a case in which interculturality played a big role, the cultural nature of the law and its language, the issue of cross-cultural equivalence, the existence of an intercultural legal discourse and, like the other authors in this corpus, the interpreter's role as an intercultural agent (which leads to the question of qualifications) or mere conduit are tackled. Finally, Hammond's investigation also presents interculturality as a case argument, as well as continuing the discussion on the interpreter's role. Interestingly, he argues that if translators/interpreters become fully-fledged interculturality experts, this would contribute to the professionalisation of their job.

All in all, the main issue related to interculturality within legal interpreting seems to be that of the interpreter's role: should s/he act as a cultural broker? In which instances? The answers to these questions require a clear definition of cross-cultural differences, which as the corpus demonstrates, still has not been agreed upon, as well as of the qualifications required to become an intercultural agent. Clarification seems to be needed on what we consider, at least in legal settings, culture and intercultural issues. The analysed corpus engages with the discussion on cross-cultural equivalences, but no unifying solution has 
been identified. Finally, it seems like the possibilities of the intercultural legal discourse have not yet been fully explored.

Before rejecting or confirming the claim posited, I must first acknowledge the limitations of this study. First of all, the corpus studied cannot be considered a representative sample of research in legal interpreting, since it is made up of only five texts. Furthermore, I only included articles which indexed interculturality as one of their keywords. However, this does not mean that other texts do not take into account this issue, since it is present in the very nature of legal interpreting. Finally, this study has been limited to English-language research, excluding other languages and (potentially) other legal and translational cultures.

Bearing all of this in mind, it is now time to examine the claim I made in the light of the results: do more recent studies approach interculturality in new and more diverse ways? This seems to be true for the corpus analysed: the newest article deals extensively with cross-cultural issues and approaches them in different ways. However, at this point, I cannot confidently confirm that this is the same for all research in interculturality within legal interpreting. This is why I only consider my claim as validated for the concrete case of this corpus. Further research would be needed to extend this claim beyond the limits of these pages.

\section{Conclusions and implications for the future}

My aim in this chapter was to present a mixed-methods approach for the study of interculturality and to prove its usefulness through a case study in legal interpreting. Even with a very limited corpus, as in this paper, this methodology has proved its usefulness: it has allowed to categorise how interculturality is taken into account in legal interpreting research and it has opened up further research questions. The approach presented, combining bibliometrics with Mayring's qualitative content analysis, can be used to gain insights not only into how much research is being produced but also into the content of said research. Furthermore, it can answer very specific research questions on how a concrete concept, such as interculturality or gender dimension, for example, is being integrated into research. Finally, it can also be used to identify unexplored research avenues. Further research is necessary to explore its full potential and to improve it. 


\section{Acknowledgements}

I would like to thank Dr Monzó-Nebot for her support, for encouraging me, for reading this chapter, and for her invaluable advice.

\section{References}

Alam, Shahidul. 2008. «Majority world: Challenging the West's rhetoric of democracy». Amerasia Journal 34 (1): 88-98. doi: https://doi.org/10.17953/ amer.34.1.13176027k4q614v5.

Ball, Rafael. 2018. Introduction to Bibliometrics: New Development and Trends. Cambridge: Chandos Publishing.

Bellis, Nicola de. 2015. «History and evolution of (biblio)metrics». In Beyond Bibliometrics: Harnessing Multidimensional Indicators of Scholarly Impact, edited by Blaise Cronin and Cassidy R. Sugimoto, 23-44. Cambridge: MIT Press.

Chowdhury, Gobinda G. 2010. Introduction to Modern Information Retrieval. 3rd ed. London: Facet.

Cooke, Michael S. 1995. «Understood by all concerned? Anglo/aboriginal legal translation». In Translation and the Law, edited by Marshall Morris, 37-63. Amsterdam: John Benjamins.

Franco Aixelá, Javier. 2001-2019. BITRA (Bibliografía de Traducción e Interpretación): Base De Datos En Acceso Abierto https://aplicacionesua.cpd.ua.es/tra_int/ usu/buscar.asp?idioma $=e n$.

Franco Aixelá, Javier. 2004. «The study of technical and scientific translation: An examination of its historical development». Jostrans - The Journal of Specialised Translation 1:29-49. https://www.jostrans.org/issue01/art_aixela.php.

Franco Aixelá, Javier. 2010. «Una revisión de la bibliografía sobre traducción e interpretación médica recogida en BITRA (Bibliografía de Interpretación y Traducción)».Panace@ 11: 151-160. https://www.tremedica.org/wp-content/ uploads/n32_tribuna_aixela2.pdf.

Franco Aixelá, Javier, and P. Orero Clavero. 2005. «Research on audiovisual translation: Some objective conclusions, or the birth of an academic field». In Research on Translation for Subtitling in Spain and Italy, edited by John D. Sanderson, 79-92. Alicante: Universidad de Alicante. 
Gambier, Yves, and Luc van Doorslaer. 2004-2019a. «General information: Criteria \& coverage». Accessed November 14, 2019. https://benjamins.com/online/ tsb.

Gambier, Yves, and Luc van Doorslaer, eds. 2004-2019b. TSB: Translation Studies Bibliography. Amsterdam and Philadelphia: John Benjamins.

Geisler, Eliezer. 2000. The Metrics of Science and Technology. Westport: Quorum Books.

Gile, Daniel. 2000. «The history of research into conference interpreting». Target 12 (2): 297-321. doi: https://doi.org/10.1075/target.12.2.07gil.

Glänzel, Wolfgang. 2003. «Bibliometrics as a research field: A course on theory and application of bibliometric indicators». https://www.cin.ufpe.br/ ajhol/ futuro/references/01 \%23_Bibliometrics_Module_KUL_BIBLIOMETRICS \%20AS \%20A \%20RESEARCH \%20FIELD.pdf.

Grbić, Nadja, and Sonja Pöllabauer. 2008. «Counting what counts: Research on community interpreting in German-speaking countries - A scientometric study». Target 20 (2): 297-332. doi: https://doi.org/10.1075/target.20.2.06grb.

Hall, Edward Twitchell. 1973. The Silent Language. Anchor books 948. New York: Anchor Press.

Harding, Sue-Ann, and Ovidi Carbonell Cortés. 2018. «Introduction: Translation and culture». In Harding and Carbonell Cortés 2018, 1-13.

Harding, Sue-Ann, and Ovidi Carbonell Cortés, eds. 2018. The Routledge Handbook of Translation and Culture. London: Routledge.

Höffe, Otfried. 2015. «Intercultural legal discourse: Philosophical aspects». In International Encyclopedia of the Social \& Behavioural Sciences, edited by James D. Wright, 362-367. Amsterdam: Elsevier.

Katan, David. 2018. «Defining culture, defining translation». In Harding and Carbonell Cortés 2018, 17-47.

Kracum, John. 2014. «The validity of United States v. Nazemian following Crawford and its progeny: Do criminal defendants have the right to face their interpreters at trial?» Journal of Criminal Law and Criminology 104 (2): 431. https:// scholarlycommons.law.northwestern.edu/jclc/vol104/iss2/5.

MACI, ed. 2016. Brief for the Massachusetts Association of Court Interpreters, INC. As Amicus Curiae in Support of Petitioner No. 15-10002. Chelmsford: Supreme Court of the United States.

Martínez-Gómez, Aída. 2015. «Bibliometrics as a tool to map uncharted territory: A study on non-professional interpreting». Perspectives 23 (2): 205-222. doi: https://doi.org/10.1080/0907676X.2015.1010550. 
Mayring, Philipp. 2004. «Qualitative content analysis». In a Companion to Qualitative Research, edited by Uwe Flick, Ernst von Kardoff, and Ines Steinke, 266-269. London: Sage Publishing.

Mayring, Philipp. 2016. Einführung in die Qualitative Sozialforschung: Eine Anleitung zu Qualitativem Denken. Weinheim, Basilea: Beltz.

Monzó-Nebot, Esther. 2010. «E-Lectra: A bibliography for the study and practice of legal, court and official translation and interpreting». Meta 55 (2): 355-373.

Monzó-Nebot, Esther. 2018. «Translation and culture in legal settings and institutions». In Harding and Carbonell Cortés 2018, 463-482.

Pöchhacker, Franz. 2011. «Researching interpreting: Approaches to inquiry». In Advances in Interpreting Research: Inquiry in Action, edited by Brenda Nicodemus and Laurie Swabey, 5-25. Amsterdam: John Benjamins.

Pöllabauer, Sonja. 2006. «During the Interview, the interpreter will provide a faithful translation. The potentials and pitfalls of researching interpreting in immigration, asylum and police settings: Methodology and research paradigms». In Taking Stock: Research and Methodology in Community Interpreting, edited by Erik Hertog and E. van der Veer, 224-29. Antwerp: Hogeschool Antwerpen.

Shlesinger, Miriam. 2009. «Crossing the divide: What researchers and practitioners can learn from one another». Translation \& Interpreting 1 (1): 1-16.

Vázquez Fernández, Ana. 2019. «El género como factor en los estudios sobre interpretación no profesional». MA thesis, Universitat Jaume I.

Vidal Claramonte, África. 2013. «Towards a new research model in legal translation: Future perspectives in the era of asymmetry». Linguistica Antverpiensia 12. https://lans-tts.uantwerpen.be/index.php/LANS-TTS/article/ download/233/208.

Yan, Jackie Xiu, Jun Pan, Hui Wu, and Ying Wang. 2013. «Mapping Interpreting Studies: The state of the field based on a database of nine major translation and interpreting journals». Perspectives 3 (21): 446-473. 
ÍNDEX 


\title{
Problemes en la traducció de musicals: estratègies i tècniques per resoldre'ls
}

\author{
Joan Alfred Noll Obiol
}

al367994@uji.es

La traducció per a la representació escènica de musicals, un gènere popular a Espanya en versió traduïda (Mateo 2008), s'ha estudiat tradicionalment des d'un marc teòric que inclou la traducció funcional (Nord 2006), la traducció de texts lligats a música (Golomb 2005) i l’aproximació Pentatló a la traducció cantable (Low 2012). El fet que els musicals traduits hagin de ser representables implica diverses limitacions difícils d'equilibrar, dominades per la dependència de la paraula amb la música i analitzables comparant diverses traduccions. Aquest procés permet revisar posteriorment la intervenció del traductor/a expert, en la forma d'idees afegides o un estil diferent en el text meta, així com les seves motivacions en una tasca tan restringida (Apter 1985). En aquest article es presenta el disseny d'un estudi que, adaptant les eines ja existents, permeti accedir a aquestes motivacions, a través de la realització i anàlisi de traduccions dels musicals Sunday in the Park with George (Sondheim i Lapine 1991) i Into the Woods (Sondheim i Lapine 1986), en versions en català i italià. Aquest estudi s'articula a partir d'una adaptació $i$ ampliació de les eines d'anàlisi textual per a la traducció de musicals adequada per als casos estudiats. La metodologia aplicada per implementar aquestes eines modificades inclou una anàlisi estructural de la composició dels texts, la comparació entre traduccions i la classificació de les estratègies i tècniques de traducció utilitzades, així com el disseny d'entrevistes als traductors, destinades a triangular els resultats obtinguts a partir de l'anàlisi textual. 
Paraules clau: musicals, problemes de traducció, traducció relacionada amb la música, Sondheim, tècniques de traducció

\section{Introducció}

El gènere anomenat musical, malgrat provenir de gèneres anteriors, tal i com opinen Gottfried i Frankel, en considerar-lo un producte de la cançó i dansa bàsiques del vodevil $(1979,7)$, es pot considerar com «una forma escènica purament americana» (ibid.). Es poden trobar obres autòctones tan aviat com el 1767, amb The Disappointment de Samuel Adler, però la cristal-lització del gènere com a popular i amb influència en la societat es donaria recentment: «[c]om a forma d'entreteniment popular per a públics prou massius, al llarg del segle XX els musicals van dramatitzar, reflectir o desafiar diversament les nostres actituds culturals i creences més arrelades $»^{1}$ (Jones 2003 , 2). Més enllà de tenir una història encara curta, es tracta d'un gènere que ha estat poc estudiat des d'una perspectiva acadèmica, malgrat la popularitat que ha adquirit i l'impacte assolit tant en la cultura on es va originar com en aquelles en què s'ha difós, amb versions originals o traduïdes.

La particularitat més remarcable dels texts de musical és que combinen música i paraula, seguint la tradició dels gèneres relacionats amb el teatre musical europeu clàssic (òpera, sarsuela, opereta i altres), tot i que afegeixen altres factors escènics a l'hora de representar-los, com la inclusió de coreografies o de muntatges escenogràfics que enriqueixen la història contada. De fet, Gottfried i Frankel ens expliquen que "[e]l musical és un art a la vegada popular i d'alta qualitat, que comparteix arrels amb el vodevil i el burlesque» $(1979,7)$. Cal remarcar que, en aquest article, ens centrem en musicals de llibre, que conten una història, en contraposició als anomenats revue compostos per una sèrie de cançons i esquetxos (ibid. 1979, 12).

En una tasca de traducció que molts cops és una empresa solitària, cada traductor/a aportarà l'experiència, les idees i l'expertesa a l'hora de confeccionar

1. Totes les traduccions de l’anglès són pròpies. 
una traducció adequada per a l'encàrrec que se li hagi fet. Lestudi de les diferències entre els texts traduïts a cultures diferents ens pot ajudar a esbrinar quines han estat les motivacions que han portat a prendre eleccions de traducció diverses. En aquest article en particular, es presenta el disseny d'un estudi que permeti accedir a aquestes motivacions i que s’articula com $a$ ) una adaptació i ampliació de les eines d'anàlisi per a la traducció de musicals adequada per als casos estudiats, incloent l'anàlisi de la composició del text, $b$ ) una comparació de traduccions i $c$ ) la implementació d’entrevistes als traductors/es.

\section{Revisió i adaptació de les eines d'anàlisi de musicals}

L'article s'emmarca dins la recerca en el camp de la traducció de musicals, concretament en la (re)definició d’eines metodològiques destinades a analitzar tant les estratègies i tècniques de traducció utilitzades pels traductors/es professionals com, a posteriori, a explicar els efectes dels texts en les societats de destí (meta) en què siguin traduïts per a la representació escènica. Els texts predecessors dels musicals, en general aquells que es consideren com a part del teatre musical clàssic europeu, com l'òpera, han estat estudiats des de diverses perspectives, entre elles la que ens ocupa dels estudis de traducció. Tot i així, la recerca en el camp és escassa. Aquest article en concret contribueix a les recerques disponibles amb la proposta d'eines d'anàlisi textual per als texts de musical, caracteritzats pel fet que la paraula i la música es troben marcadament enllaçades.

Des de la definició dels subtipus de text inclosos en un musical, s'efectua una primera aproximació a les unitats de traducció utilitzades per subdividir l'anàlisi comparativa. A partir d'aquesta subdivisió, s'ha fet una descripció de les estratègies de traducció usades en la tasca de traducció. La subseqüent comparació de traduccions representables de musicals realitzades per professionals, un cop redefinit el mètode d'anàlisi textual, ens ha de servir per detectar les solucions aplicades per als problemes de traducció fonamentals d'aquests texts, atesa la seva dependència del ritme musical. Les tècniques utilitzades per a cada subtext s'han etiquetat segons la classificació d'Hurtado i Molina (taula 1).

El disseny proposat es completa amb entrevistes semi-estructurades destinades a traductores/es professionals que permetran triangular les dades obtingudes, aportant un altre origen de dades a l'anàlisi de les traduccions i, per tant, donant una major validesa a les conclusions obtingudes, amb una metodologia 
mixta. El fet de poder parlar amb els autors/es d'aquestes ens ofereix la possibilitat de comprovar, refutar o enriquir les conclusions obtingudes a partir de la comparació textual, quant a les eleccions de tècniques de traducció, en el cas del musical Into the Woods, i conèixer de primera mà les estratègies utilitzades en realitzar les seves traduccions enllaçades amb la música en un entorn professional.

La revisió d'aquestes eines hauria de permetre la seva utilització en recerques posteriors, i ajudar així al foment de la recerca en aquest camp de la traducció tant interessant com poc estudiat.

\section{Traducció de musicals per a escena}

Els texts utilitzats en aquesta recerca són els musicals Sunday in the Park with George (Sondheim/Lapine 1984) i Into the Woods (1986), dels mateixos autors. Pel que fa a Into the Woods, es compararan les traduccions al català (Joan Vives) i a l'italià (Andrea Ascari) per esbrinar les solucions trobades per aquests dos traductors subjectes a tradicions culturals diverses i amb diferent pes demogràfic $i$ influència.

La teoria sota la qual s'analitzen els materials s'emmarca en la traducció funcional (Reiss i Vermeer 1971) i en la visió anomenada funció més fidelitat (Nord 2006). La metodologia de traducció proposada per Nord per realitzar traduccions a partir del seu Skopos o objectiu es pot prendre com a base a l'hora d'analitzar traduccions, veient si el producte final compleix els seus objectius, habitualment proveïts per un encàrrec de traducció.

En el cas concret dels texts de musical per a escena, el tipus de traducció estudiada s'anomena «equifuncional», ja que es pretén que les traduccions facin el mateix paper en la cultura meta que el que feien en la cultura origen, és a dir: s'han de produir texts que també siguin interpretables en un escenari en el llenguatge meta.

Alguns dels problemes que presenta la traducció d'aquests materials per a escena són els mateixos que en qualsevol tipus de traducció teatral, ja que els texts han de ser interpretables. Franzon ho expressa dient que «els professionals del teatre poden preferir una traducció 'representable' d'una obra que una de 'literària'» (Franzon 2008, 375). En aquest cas, a més, s'afegeix la dependència respecte de la música, fent que sigui el traductor/a qui ha de «modificar la 
representació verbal, plantejant-la més lliurement, parafrasejant o esborrant-ne parts i afegint al contingut de la lletra [musical] original. (ibid., 386)». Fins a quin punt es tradueixen les referències culturals o es mantenen les originals? Es conserva el to i l'estil de l'obra o s'adapta aquest als costums de la cultura meta per a aquest tipus de texts? Aquestes preguntes i moltes altres de similars han estat estudiades en la traductologia i en molts casos s'han utilitzat per qualificar una traducció com a encertada o errònia, com a fidel o infidel al text origen. Tanmateix, hem de tenir en compte que, quant al fet que els texts comparats han estat ja representats en les cultures meta i en alguns casos amb un èxit considerable, aquest fet ja es pot considerar com una mostra que la traducció ha estat adequada per als seus fins, encara que per a reeixir s'hagi hagut d'allunyar de loriginal en major o menor mesura. Dit això, la traducció de textos enllaçats amb la música presenta tota una sèrie de problemes relacionats amb la dependència del text a la música i que cal tenir en compte per analitzar els canvis en la traducció i les seves causes últimes.

El tipus de traducció concret que tracta amb texts que inclouen paraula i música fou etiquetat per Golomb com Traducció Enllaçada amb la Música (o TEM) (2005), terme que expressa sintèticament tant el contingut com la problemàtica que impliquen aquests tipus de texts a l'hora de traduir-los. Tot i que existeixen diversos tipus de traducció en aquest àmbit, depenent entre altres coses del grau de sincronització entre la paraula i la música, el tipus que es tracta en aquesta recerca és aquell que implica major sincronització. Així, la traducció interpretable sobre un escenari del text d'un musical ha de conservar el mateix contingut musical de l'original, però ha de produir un text meta tan proper a l'original com sigui possible, depenent de la llibertat donada en l'encàrrec, i a la vegada sincronitzar aquestes paraules amb la mateixa música en les parts del text on es doni aquesta dependència.

En analitzar el text d'un musical, per tant, ens trobem amb un material que inclou «dos sistemes auditius separats, el musical i el verbal» com ja ho explicava Apter en el seu article fundacional en el camp sobre les dificultats de traduir òperes a l'anglès $(1985,309)$. Malgrat les similituds amb el cas estudiat, també cal tenir en compte les diferències, ja que Apter parlava de traduccions que havien d'incloure, en la major part dels casos, versos rimats. Els musicals, i més concretament els estudiats en aquest treball, tenen de fet alguns versos rimats però aquest tipus de material no és ni molt menys el més comú. Els mètodes usats per analitzar el text seran: l'anàlisi textual, en la seva definició modificada 
per al tipus concret de text a partir de les troballes del màster, seguit per l'anàlisi comparatiu entre traduccions representades sobre un escenari.

Lanàlisi de la primera escena del musical ofereix una sèrie de resultats inicials sobre els quals es poden elaborar les primeres conclusions. Per tal d'analitzar la part verbal del text, s'utilitza un conjunt de tècniques fonamentades en la classificació d'Hurtado i Molina (2002/2011), basada en una llarga tradició dins el camp de la traducció amb aportacions de teòrics com Vinay i Dalbernet, Nida o Newmark, entre d'altres.

Taula 1. Tècniques de traducció segons Hurtado i Molina

Tècnica

Adaptació $[\mathrm{AD}]$

Ampliació lingüística [AL]

Amplificació [AM]

Reducció [RE]

Calc $[\mathrm{CA}]$

Compensació $[\mathrm{CO}]$

Compressió lingüística [CL] Sintetització d’elements lingüístics

Creació Discursiva [CD]

Descripció [DE]

Equivalent encunyat $[\mathrm{EE}]$
Canvi d'elements culturals

Addició d’elements lingüístics

Addició de precisions no formulades al text origen

Pèrdua de precisions

Traducció literal de terme estranger

Introducció d'element d'informació en un altre lloc del text

\begin{tabular}{lc} 
Descripció $[\mathrm{DE}]$ & funció \\
\hline Equivalent encunyat $[\mathrm{EE}]$ & $\begin{array}{c}\text { Utilització d'equivalent reconegut per l'ús o el } \\
\text { diccionari }\end{array}$
\end{tabular}

Generalització [GE] Ús d’un terme més general i neutre 


\section{Tècnica}

Canvi de punt de vista, enfoc o categoria (lèxic/
estructural)

\section{Definició}

Ús d’un terme més precís o concret
Ús de paraula o expressió d’una altra llengua
Préstec [PR]

Canvi d’elements lingüístics per paralingüístics o

Substitució [SU] viceversa

\begin{tabular}{cc} 
viceversa \\
\hline Traducció literal [TL] & $\begin{array}{c}\text { Traducció paraula per paraula d’un sintagma o } \\
\text { expressió }\end{array}$
\end{tabular}

Transposició [TR] Canvi de categoria gramatical

Variació [VA] Canvi d'elements lingüístics o paralingüístics

Per a Hurtado, el terme mètode de traducció es refereix a la forma general en què un text es tradueix, que en aquest cas es podria resumir com una traducció amb una intenció interpretativa-comunicativa, que malgrat els canvis de contingut, traslladi efectivament el missatge del text original. A més, cal diferenciar també entre estratègies i tècniques de traducció, que per a cada unitat de traducció «descriuen el resultat obtingut i poden ser usades per classificar diferents tipus de solucions traductives», mentre que les estratègies es refereixen als «mecanismes usats pels traductors al llarg del procés de traducció complert» (Hurtado i Molina 2002, 507). Per resumir, les tècniques disponibles, emmarcables també dins el contínuum de tècniques de Marco (2004, 3-4), que en permet la superposició dins una unitat de traducció, es relacionen en la taula 1 , indicant l'etiqueta utilitzada per indicar la tècnica detectada en cada cas, i incloent tècniques tant lingüístiques com culturals, segons la classificació d'Hurtado/Molina.

Cal tenir en compte que, per les particularitats del text, on la paraula parlada es troba enllaçada amb el ritme musical, és d'esperar un nombre elevat d'unitats traduïdes mitjançant l'ús de la creació discursiva, així com el moviment d’idees i conceptes d'un vers a un altre, per conservar-les malgrat les restriccions formals. 
Per últim, i tal com defineix Marco (2004, 138), es considera que l'equivalent encunyat, quan ens estem referint a la unitat de traducció del text de musical, es pot considerar com un nivell de traducció zero, ja que més que solucionar un problema en aquests casos s'està utilitzant una solució acceptada i que, per tant, inicialment es consideraria com la solució estàndard, i que se situa fora del conjunt de tècniques d'Hurtado i Molina.

Un cop revisada la part verbal no podem deixar de tenir en compte la relació d'aquesta amb la part musical, per a la qual cosa s'adaptarà l'anomenat mètode Pentatló de Low $(2005,191-199)$ a l'ús amb el text de musicals. Així, tot i que per a Gorlée l'enfocament musico-cèntric en la traducció de cançó significaria no tenir en compte el text parlat (Gorlée 2005, 8), en aquest cas implica tan sols que es troba totalment supeditat a la música. Per a Low, la traducció de texts que enllacen la música i la paraula, en una visió bàsicament musicocèntrica que en suposa la preponderància, els cinc paràmetres que permeten comprovar la idoneïtat d'una traducció per a ser cantada són el significat, el ritme, la rima, la naturalitat i la cantabilitat. L'adequada ponderació d'aquests cinc paràmetres permet assolir una traducció cantable el més compensada possible, sempre que les que en el seu enfocament considera pèrdues en la traducció siguin ben repartides entre els cinc paràmetres.

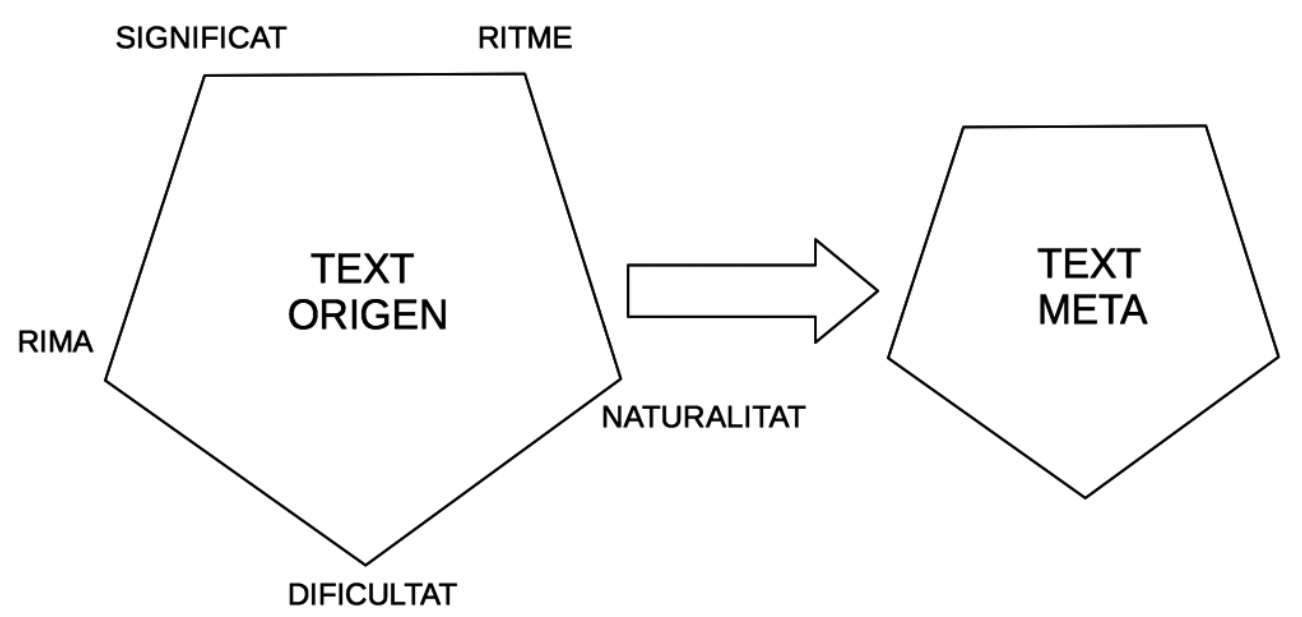

Figura 1. Representació d’una traducció cantable equilibrada 
La figura 1 mostra gràficament (indicant-ho amb la reducció del radi per a cada vèrtex del pentàgon, corresponent a cada paràmetre), una representació de com una traducció cantable pot sofrir pèrdues en els diversos paràmetres (segons el principi Pentatló de Low) i tot i així seguir sent equilibrada. Per la seva part, en la figura 2 podem veure representada la possibilitat que la traducció cantable hagi sofert pèrdues de manera desigual. Per exemple, en el cas que s'hagi mantingut el ritme però el significat no hagi estat tingut tant en compte, $i$ altres paràmetres hagin també canviat en ponderacions diverses.

En la figura 3, d'altra banda, podem observar un exemple del tipus de material amb què cal treballar per analitzar el text origen i les seves traduccions. El material, contingut en la partitura vocal i destinat a la preparació de la representació per als intèrprets de la peça, inclou tots els tipus de subtext en què s'ha subdividit el material, tal com es detallarà en el proper apartat.

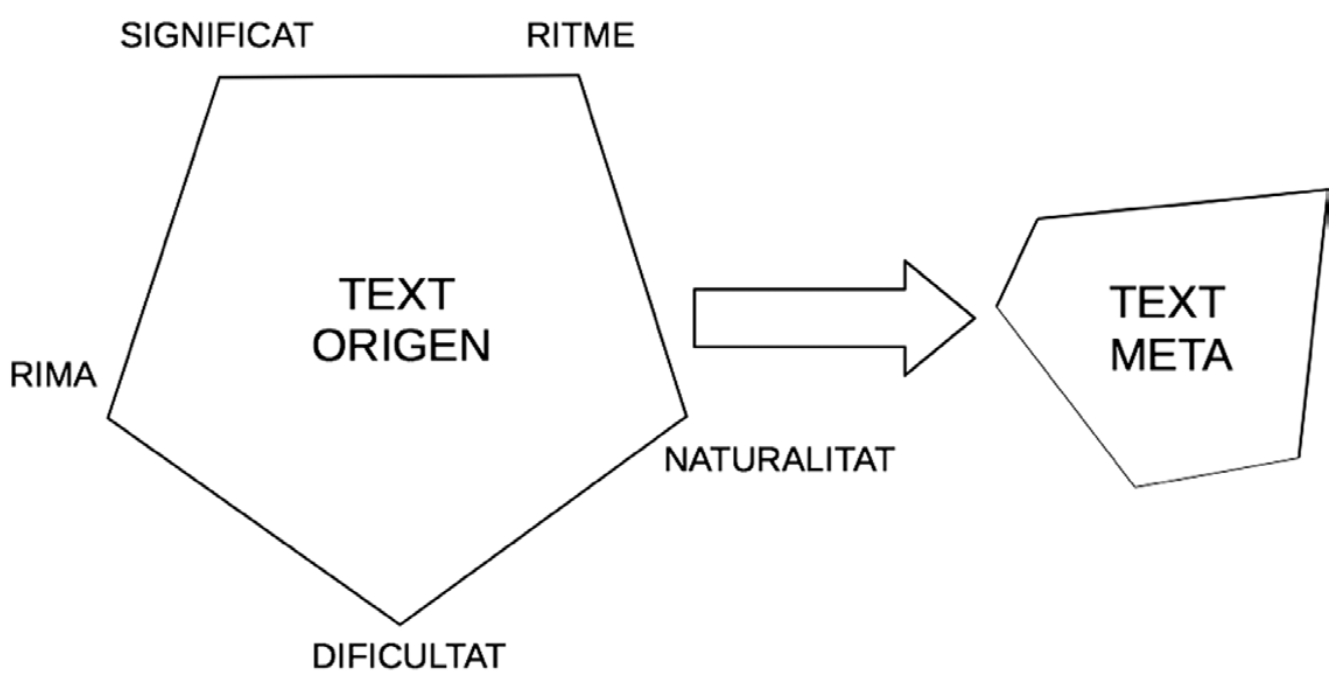

Figura 2. Representació d'una traducció cantable no equilibrada 


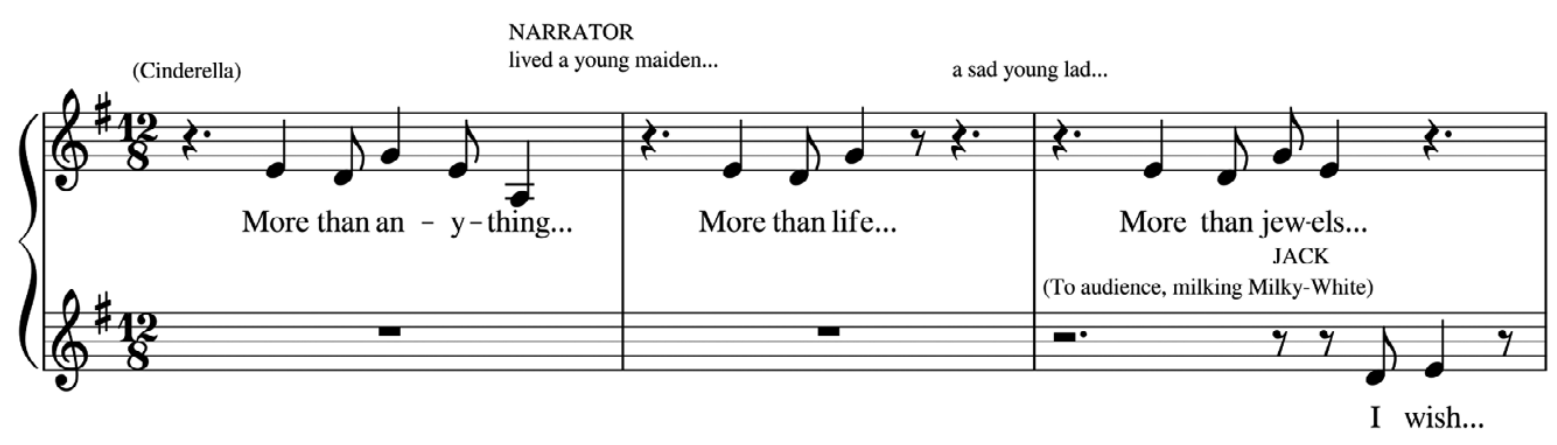

Figura 3. Fragment d'Into the Woods (Vocal Score), p. 211

\section{Resultats i anàlisi: Les traduccions d'Into the Woods}

El treball inicial amb els materials de Sunday in the Park va permetre subdividir el text del musical segons els subtipus de contingut verbal detectats. Lobservació d’altres musicals pertanyents al mateix gènere, objectiu més enllà de làmbit d’aquest article, hauria de permetre demostrar que aquesta subdivisió és generalitzable per al tipus de text estudiat. Així, es pot considerar que els tres subtipus de text que apareixen són la paraula parlada, la paraula cantada i les indicacions escèniques/teatrals. Com a exemple del procés seguit, es mostra a continuació l’anàlisi del principi de la primera escena del musical Into the Woods, que inclou paraula parlada («in a far off kingdom...»), paraula cantada («I wish... More than anything...») i indicacions teatrals o escèniques (To audience, polishing floor). Per tant, resulta adequat per exemplificar el mètode d’anàlisi implementat en la comparació de traduccions.

Les restriccions imposades per la sincronització amb la música permeten argumentar que el text cantat serà el més dificultós de traduir, mentre que el text parlat malgrat haver de respectar la coherència amb els canvis implementats en el text cantat presentarà menys restriccions formals. Finalment les indicacions escèniques haurien d’oferir llibertat màxima en la traducció, donat que són els texts que s'utilitzaran per a la preparació prèvia de l'escenari i la interpretació dels/les intèrprets. Aquestes hipòtesis ens permeten considerar que la traducció del text s'haurà realitzat estratègicament seguint l'ordre «Text cantat - Text parlat - Indicacions escèniques», de manera que els canvis deguts a la traducció enllaçada a la música es puguin compensar en les traduccions subsegüents. 
En les taules 2 i 3 observem els exemples de cada tipus de traducció segons el subtipus de text ( $\mathrm{IE}=$ Indicacions escèniques, $\mathrm{PP}=$ Paraula parlada, $\mathrm{PC}=$ Paraula cantada), per als idiomes català i italià. Tot i que evidentment no es tracta d'una mostra representativa de com de les tècniques són aplicades en la totalitat del text, sí que ens permet observar el mètode utilitzat per comparar el text original i el text meta a l'hora d'extreure'n dades en brut.

Taula 2. Traducció interpretable al català

\begin{tabular}{cccc} 
Tipus & Text origen & Text meta - català & $\begin{array}{c}\text { Tècnica } \\
\text { de traducció }\end{array}$ \\
IE & $\begin{array}{c}\text { (Light on Jack and Milky- } \\
\text { White) }\end{array}$ & $\begin{array}{c}\text { Llum sobre JAN i } \\
\text { la vaca }\end{array}$ & $\begin{array}{c}\text { Equivalent } \\
\text { encunyat }\end{array}$ \\
\hline PP & ...a young maiden... & ...una donzella... & $\begin{array}{c}\text { Equivalent } \\
\text { encunyat }\end{array}$ \\
\hline PC & More than an-y-thing & Per da-munt de tot & $\begin{array}{c}\text { Equivalent } \\
\text { encunyat }\end{array}$ \\
\hline
\end{tabular}

Cal remarcar que, en els dos idiomes, la traducció del text parlat en aquest cas utilitza la tècnica de l'equivalent encunyat. Es pot considerar que també ho fa a nivell lingüístic per a les indicacions escèniques, encara que es perd el nom de la vaca (Milky-White) en els dos idiomes meta.

Per últim podem veure com la traducció en català del text cantat també aconsegueix conservar el significat del text original amb un equivalent encunyat, cosa que no succeirà en la major part del text, mentre que en italià ha calgut realitzar una transposició, utilitzant la traducció d'un altre vers ( «I wish») en aquesta unitat de traducció particular. En una visió més enllà de l'exemple mostrat, cal remarcar que -a més dels resultats pròpiament tècnics- l'anàlisi comparativa de la primera escena ens mostra com el text català presenta característiques estilístiques diferencials com l'ús d'abreviacions o l'addició de tocs d'humor no existents a l'original o la traducció italiana (per exemple en l'escena on s'explicita que el malefici llençat per la bruixa al forner és destinat a les seves «parts»). 
Taula 3. Traducció interpretable a l'italià

\begin{tabular}{cccc} 
Tipus & Text origen & $\begin{array}{c}\text { Text } \\
\text { meta }- \text { italià }\end{array}$ & $\begin{array}{c}\text { Tècnica } \\
\text { de traducció }\end{array}$ \\
IE & $\begin{array}{c}\text { (Light on Jack and Milky- } \\
\text { White })\end{array}$ & $\begin{array}{c}\text { Luci su Jack e la } \\
\text { mucca }\end{array}$ & $\begin{array}{c}\text { Equivalent } \\
\text { encunyat }\end{array}$ \\
\hline PP & ...a young maiden... & ...una fanciulla... & $\begin{array}{c}\text { Equivalent } \\
\text { encunyat }\end{array}$ \\
\hline PC & More than an-y-thing & Io de-si-de-ro & Transposició \\
\hline
\end{tabular}

Així, després d'analitzar la primera escena d'Into the Woods en la seva traducció a l'italià i al català, els primers resultats quant a les tècniques de traducció utilitzada són els següents:

- El text cantat, atesa la seva elevada restricció deguda a la música, ha estat traduït en un elevat nombre de casos utilitzant la tècnica de la creació discursiva i la compensació, tal com es preveia. Malgrat això, és remarcable el fet que en la traducció italiana els significats són molt més propers a loriginal i per tant amb un menor nivell de creació discursiva.

- El text parlat, que s'havia considerat en un segon nivell de dificultat i que per tant seria susceptible de canviar a causa de la traducció del text cantat, s'ha traduït en ambdós idiomes utilitzant majoritàriament traducció de nivell zero, o equivalent encunyat.

- Les indicacions escèniques, que també s'havien suposat com a susceptibles de canvi per compensar les modificacions degudes a la traducció enllaçada amb la música, també mostren en la major part dels casos traducció de nivell zero.

La revisió d'aquestes dades permet afirmar que de fet els supòsits inicials sobre quins subtexts canviarien més en la traducció no es compleixen en aquests dos casos. Per tant les estratègies hipotetitzades per a la traducció d'aquest tipus de text, quant a ordre de traducció i enfocament de cada subtipus, no haurien estat necessàriament aplicades en la traducció realitzada per professionals. Quant 
al text cantat, és cert que es produeixen un gran nombre de versos amb creació discursiva, compensació i altres tècniques com la generalització i la particularització però, aparentment, tots aquests canvis que es donen en la traducció del subtext cantat no han afectat necessàriament els altres subtipus de text.

Un cop analitzats els components lingüístics, però, cal tenir també en compte els components musicals per a aquest cas. Aplicant a l'exemple analitzat els cinc paràmetres definits per Low en la seva aproximació Pentatló a la traducció cantable (tot i que modificant el nom del cinquè paràmetre cantabilitat pel de dificultat que al meu parer defineix millor el component mesurat pel paràmetre) ens trobem amb els resultats mostrats en la taula 4 . La taula correspon exclusivament al subtipus de text corresponent a la paraula cantada.

Taula 4. Mostra del grau de similitud entre les traduccions cantables i l'original

Text origen Text meta Significat Ritme Rima Naturalitat Dificultat

$\begin{array}{cccccc}\begin{array}{c}\text { More than } \\ \text { anything }\end{array} & \begin{array}{c}\text { Per } \\ \text { damunt } \\ \text { de tot }\end{array} \quad \text { Alt } & \text { Alt } & \text { N/E } & \text { Alt } & \text { Alt }\end{array}$

\begin{tabular}{ccccccc}
\hline $\begin{array}{c}\text { More than } \\
\text { anything }\end{array}$ & $\begin{array}{c}\text { Io } \\
\text { desidero }\end{array}$ & $\begin{array}{c}\text { Alt } \\
\text { (Transp.) }\end{array}$ & Alt & N/E & Alt & Alt \\
\hline
\end{tabular}

En la taula s'indica el grau de similitud dels components segons una gradació qualitativa dividida en (Alt/Mitjà/Baix/No Existent), considerant un grau encara que el text s'hagi transposat. Com hi podem observar, es mantenen de manera notable tots els paràmetres del mètode Pentatló, aconseguint així una traducció adequada a la seva finalitat.

Un cop revisats els components lingüístics i els musicals, es poden resumir les troballes afirmant que la paraula cantada, a més de conservar la cantabilitat, comporta un ús notable de la creació discursiva, mentre que tant les indicacions escèniques com el text parlat s'han traduït en català i italià utilitzant com a tècnica general la traducció de nivell zero, donat que els termes s'han traduit generalment als seus equivalents més acceptats. Per què es dona aquesta fixació del text a priori menys restrictiu? És possible que es tracti d'una imposició de 
l'encàrrec de traducció? Potser els traductors com a experts han pres la mateixa decisió en ambdós casos? En aquest punt és quan sembla necessària l'aportació de noves dades per revisar els resultats parcials obtinguts.

Laddició d'un mètode etnogràfic com la realització d'entrevistes als traductors ens pot portar a entendre millor les tries que aquests experts han implementat en les seves tasques, a la vegada que ens permet triangular les dades obtingudes. El procés previ d'anàlisi textual n’ofereix la base sobre la qual dissenyar nous instruments d'anàlisi sociològica, per tal com aporta:

- Una relació d'estratègies i tècniques utilitzades per traductors professionals en la traducció de musicals.

- Laparició de noves preguntes de recerca basades en els resultats obtinguts, verificables fins a un cert punt mitjançant l'ús d’altres mètodes de recollida de dades.

Aquests resultats, conjuntament amb la utilització del disseny d'entrevistes semi-estructurades basat en Seidman (2006, 17-19), possibiliten eines de recollida adequades, tot i notant la necessitat de tenir la capacitat de modificar les preguntes durant l'entrevista (repreguntar), en base a un bon coneixement $\mathrm{i}$ l'experiència personal sobre el tipus de traducció tractada. Les bases d'aquestes entrevistes es poden plantejar segons l'estructura següent en tres parts proposada per Seidman (ibid.):

- Experiència personal de l'entrevistat/da en la seua vida personal i professional.

- Revisió detallada de la traducció realitzada, en el context de l'encàrrec de traducció. Qüestions sobre les tècniques de traducció detectades amb l'anàlisi textual.

- Reflexió personal de l'entrevistat/da sobre l'experiència de traducció en general. Qüestions sobre les estratègies de traducció utilitzades en l'àmbit professional.

Finalment, a partir d'aquesta segona sèrie de resultats obtinguts mitjançant les entrevistes es podrà comprovar fins a quin punt les estratègies extretes a partir de la realització d'una traducció i l'anàlisi textual es poden considerar adequades en un o diversos casos, o si per contra, com sembla suggerir la comparació de la traducció catalana i la italiana, aquestes estratègies no són sempre necessàries o en tot cas universals. Les entrevistes també poden aportar respostes 
per saber fins a quin punt el traductor/a farà la feina amb la intenció de deixar la seva empremta o només de fer asèpticament la feina assignada, així com per saber quines tècniques i estratègies de traducció considera adequades per a la traducció de musicals per a escena.

\section{Conclusions}

Lestudi dels texts i les seves corresponents traduccions interpretables ha permès desenvolupar un mètode per a l'anàlisi de la traducció del text musical basat en la combinació de l'anàlisi de traduccions segons la classificació definida per Hurtado/Molina, complementada amb el mètode Pentatló de Low, que afegeix les consideracions degudes a la dependència amb la música.

Mitjançant l'anàlisi i la comparació de traduccions d'Into the Woods s'han detectat en una primera aproximació diferències d'estil i enfocament de la traducció que es podran aprofundir en propers estadis de la investigació, un cop classificades les tècniques de traducció utilitzades en cadascuna de les traduccions. Aquesta aproximació ofereix resultats com l'observació que en el cas català s'ha intentat assolir un text amb un to més humorístic i més familiar a l'audiència meta, omplint així un buit en el sistema cultural, tal com ja suggeria Mateo parlant de musicals traduits a l'espanyol (2008).

Quant a la determinació del mètode i estratègies de traducció, seguint la classificació d'Hurtado/Molina, la primera aproximació realitzada mitjançant una traducció i comentari ha ofert una sèrie de resultats detallats en l'article, però cal remarcar que les traduccions realitzades per traductors professionals no s'hi ajustarien sempre, segons l'anàlisi comparativa dels materials disponibles. En aquest sentit, el disseny d'entrevistes semi-estructurades proposat ha de permetre revisar els resultats i conclusions inicials, a partir de la valuosa font d'informació de primera mà que suposa l'experiència dels traductors/es.

Per finalitzar, com a ampliació de la recerca, i tenint en compte l'escassetat actual de traductors/es professionals de musicals i l'increment d'espectacles en versió original, es considera la possibilitat de realitzar també entrevistes a cantants de musical. Aquests poden aportar una visió experta sobre la representació dels texts, i de fet, en alguns casos, el procés de traducció s'haurà realitzar amb el suport de la companyia teatral en conjunt. A més, els/les cantants són qui poden indicar de manera adequada fins a quin punt la traducció ha preservat o 
modificat la dificultat per representar el text origen, segons el paràmetre indicat en el principi Pentatló de Low com a cantabilitat.

\section{Bibliografia}

Apter, Ronnie. 1985. «A peculiar burden: Some technical problems of translating opera for performance in English». Meta 30 (4): 309-319.

Bosseaux, Charlotte. 2011. «The translation of song». A The Oxford Handbook of Translation Studies, editat per Kirsten Malmkjær i Kevin Windle, 132-141. Òxford: Oxford University Press.

Franzon, Johan. 2008. «Choices in song translation: Singability in print, subtitles and sung performance». The Translator 14 (2): 373-399.

Golomb, Harai. 2005. «Music-linked translation (MLT) and Mozart's operas: Theoretical, textual and practical perspectives». A Song and Significance: Virtues and Vices of Vocal Translation, editat per Dinda L. Gorlée, 121-162. Àmsterdam: Rodopi.

Gorlée, Dinda. L. 2005. Songs and Significance: Virtues and Vices of Vocal Translation. Àmsterdam, Nova York: Rodopi.

Gottfried, Martin i Lory Frankel. 1979. Broadway Musicals. Nova York: HN Abrams.

Hurtado, Amparo. 2011. Traducción y traductología. Bilbao: Cátedra.

Jones, John Bush. 2004. Our Musicals, Ourselves. A Social History of the American Musical Theatre. Líban: UPNE.

Low, Peter. 2005. «The pentathlon approach to translating songs». A Song and Significance. Virtues and Vices of Vocal Translation, editat per Dinda L. Gorlée, 185-212. Àmsterdam i Nova York: Rodopi.

Marco, Josep. 2004. «Les tècniques de traducció (dels referents culturals): retorn per a quedar-nos-hi». Quaderns. Revista de traducció 11: 129-149.

Mateo, Marta. 2008. «Anglo-American musicals in Spanish theatres». The Translator 14 (2): 319-342.

Molina, Lucía i Amparo Hurtado. 2002. «Translation techniques revisited: A dynamic and functionalist approach». Meta 47 (4): 498-512.

Nord, Christiane. 2006. "Translating as a purposeful activity: A prospective approach». Teflin Journal 17 (2): 131-143. 
Reiss, Katharina. 1971[2003]. «Type, kind and individuality of text. Decision making in translation». A The Translation Studies Reader, editat per Lawrence Venuti i Mona Baker, 160-171. Londres i Nova York: Routledge.

Reiss, Katharina i Vermeer, Hans J. 2014. Towards a General Theory of Translational Action. Skopos Theory Explained. Londres i Nova York: Routledge.

Seidman, Irving. 2006. Interviewing as Qualitative Research: A Guide for Researchers in Education and the Social Sciences. Nova York: Teachers College Press.

Sondheim, Stephen i James Lapine. 1991. Sunday in the Park with George. Milwaukee: Hal Leonard Corporation.

Toury, Gideon. 1995. Descriptive Translation Studies and Beyond. Àmsterdam i Filadèlfia: John Benjamins. 
ÍNDEX 


\title{
El adorno en estuco en la Valencia de $\mathbf{1 7 0 0 .}$ De las propuestas autóctonas a la eclosión de la profesión
}

\author{
Gaetano Giannotta \\ al377467@uji.es
}

Entre la segunda mitad del siglo XVII y los comienzos de la centuria siguiente, en la ciudad de Valencia, se produjo un cambio fundamental en la práctica de la ornamentación. Con la llegada de nuevas influencias desde el extranjero, la actividad de adornar interiores eclesiásticos y particulares, que antes estaba sujeta al control arquitectónico de tradicional matriz gremial, pasó a manos de profesionales no vinculados con la propia arquitectura. Además de representar un cambio en el desarrollo de la profesión ornamental, estos artistas introdujeron en el medio valenciano nuevas técnicas, materiales y métodos de ejecución, implantaron novedosos modelos estilísticos y, por primera vez, atribuyeron al adorno un verdadero protagonismo en la definición de los programas iconográficos. Una metodología histórico-científica, la investigación archivística y bibliográfica, el análisis iconográfico y el continuo estudio directo de las obras han permitido lograr los resultados esperados. En particular, se ha conseguido hacer hincapié sobre las técnicas, métodos de ejecución y modelos estilísticos propios del adorno; profundizar en sus vinculaciones con la producción autóctona anterior y, sobre todo, con las innovaciones procedentes del extranjero; aclarar autorías dudosas, aquilatar las biografías de artistas poco conocidos y desvelar programas iconográficos inéditos. En conclusión, el presente trabajo aporta un avance en el conocimi- 
ento de la producción ornamental en Valencia que, en el setecientos, se abría artística y culturalmente a Europa.

Palabras clave: adorno, arquitectura, estuco, siglo XVIII, Valencia

\section{Introducción}

Con el presente artículo, se pretende profundizar en el conocimiento de la profesión y práctica de la ornamentación en estuco en Valencia y su entorno entre 1693 y la década de los setenta del siglo XVIII, analizando la obra tanto de los artistas más destacados cuanto de los considerados menores, así como de los talleres y escuelas. Adquiere particular relevancia en el estudio el análisis de los modelos que manejaron, entre los cuales se da importancia a los antecedentes locales pero, sobre todo, a los innovadores procedentes del resto de Europa que incorporaron elementos de una cultura artística alóctona. Además, se analizan las técnicas y métodos de ejecución empleados, la evolución de la profesión del ornamentista y su relación con lo arquitectónico. Finalmente, se aclara el protagonismo del estuco y del adorno en general en la definición de los programas iconográficos diseñados para los edificios barroquizados en la centuria de referencia.

Para conocer la profesión, se estudia de manera comparada la realidad valenciana con la europea, pero específicamente con la de Italia, donde la actividad de los estucadores experimentó un desarrollo similar con particular protagonismo de la región lombarda y de la zona del Valle de Intelvi. El fin último de esta contribución, además de aquilatar las biografías y producción de artistas eminentes del setecientos valenciano, es determinar las causas y consecuencias del formidable desarrollo del adorno y la profesión de ornamentista en la Valencia del siglo XVIII, explicar sus fases, fuentes estilísticas y metodologías y acotar, en conclusión, los motivos de su progresiva disolución a partir de la fundación de la Real Academia de Bellas Artes de San Carlos en 1768.

Para alcanzar los resultados de la presente investigación, se ha unido al trabajo de campo y a la observación directa de las obras el continuo estudio bibliográfico y la investigación de archivo. Para la elaboración de la bibliografía se han aprovechado los fondos de las bibliotecas de la Comunidad Valenciana 
y en particular de las bibliotecas de la Universitat Jaume I, de la Universitat de València y la Biblioteca Valenciana Nicolau Primitiu. Además, he utilizado los fondos de la Biblioteca Hertziana de Roma, que son insuperables respecto a la historia del arte italiano y europeo. Para profundizar en el ámbito siciliano, he empleado los fondos de la Biblioteca Central de la Región Sicilia y de la Universidad de Palermo, mientras que para Serpotta y su taller se han tomado como referencia las publicaciones del Museo Diocesano de Palermo.

Por lo que concierne las fuentes de archivo, se ha investigado la documentación notarial emitida a lo largo del siglo XVIII conservada, en parte, en el Archivo del Reino de Valencia y, sobre todo, en el Archivo de Protocolos Notariales del Patriarca de Valencia. Además, han sido objeto de investigación el Archivo de la Real Academia de Bellas Artes de San Carlos y el Archivo Municipal de Valencia.

Una puntual recogida de datos y catalogación ha acompañado la investigación junto a una permanente revisión de los datos obtenidos según premisas metodológicas histórico-críticas e histórico-culturales.

\section{El adorno en Valencia en el siglo XVII: entre las propuestas autóctonas y el cambio de gusto de finales del siglo}

\section{Breve estado de la cuestión}

Las publicaciones disponibles hasta la fecha se han ocupado de la decoración en estuco y, en general, de la decoración barroca en la ciudad de Valencia solo de manera indirecta, al tratar la arquitectura. Esto es, en las ocasiones en que se han estudiado los artistas más eminentes del entorno valenciano, se analizó además la decoración, si estos también se habían comprometido con ella, al realizar sus proyectos y los materiales y modelos que emplearon. En ese sentido, siempre se ha vinculado toda producción decorativa únicamente con antecedentes locales (Pingarrón 1998, 109-120). Al contrario, cuando la decoración había sido subcontratada a artistas considerados menores o incluso artífices anónimos, esta no fue objeto de atención por parte de los investigadores.

En realidad, todo ello es una consecuencia directa de la condición en la cual se hallaba la decoración en los años próximos al 1700, puesto que siempre estaba sometida a la propia producción arquitectónica. De hecho, tradicionalmente, la realización de 
obras arquitectónicas estaba sujeta al preponderante papel de los gremios. En el caso del adorno, los obrers de vila se adjudicaban las obras, creaban sus trazas y, por su parte, subcontrataban la realización material a los pedrapiquers; así que los primeros corresponden de algún modo a los modernos arquitectos y los segundos eran artesanos que ejecutaban un proyecto dado. Cuando las obras contenían adorno, normalmente, también se subcontrataba a los pedrapiquers. En consecuencia, la decoración estaba fuertemente vinculada a la ordenación establecida por el arquitecto y el propio adornista no tenía libertad artística (González Tornel 2004, 121-123; González Tornel 2005a, 305-306; González Tornel 2008, 121; Pingarrón 1998, 115-122).

\section{Los intentos novadores}

Dentro de este panorama, no faltaron intentos de renovación promovidos por los mayores arquitectos de la época. Entre ellos, destacó Juan Pérez Castiel (1650-1707). A menudo este maestro de obra fue contratado para renovar, mediante la decoración, espacios sagrados, que revistió sistemáticamente con mármol e incluso estucos y yesería (Aldana Fernández 1968, 55-87; Pingarrón 1998, 291-300). ${ }^{1}$ En su obra maestra, la renovación del presbiterio de la Catedral de Valencia, empleó elementos vegetales, mancebos y putti para rodear el espacio más sagrado de la Seo (figura 1). Vinculada por diferentes autores con el Churriguerismo castellano y lo andaluz, el presbiterio de la Seo pronto se convirtió en el mayor ejemplo del nuevo decorativismo barroco (Bérchez 1993, 2834; González Tornel 2005a, 33-40; Pingarrón 1998, 109-120).

Activo entre 1673 y 1703, también el arquitecto Francisco Padilla recubrió sus interiores eclesiásticos con estucos de elevadísima calidad. ${ }^{2}$ Su figura, erróneamente,

1. Aparte de la renovación del presbiterio de la Seo valenciana, Juan Pérez Castiel realizó también la reedificación de la Arciprestal de Nuestra Señora de los Ángeles de Chelva, de los templos de Tuéjar, Torrent y La Vall de Almonacid, la Capilla de Santa Bárbara en la Iglesia de San Juan del Hospital, la parroquial de San Valero en Ruzafa, la remodelación de la Iglesia de Santa Catalina de Alzira, la Capilla de la Comunión de la parroquial de Biar, además de intervenciones en la Capilla de San Pedro, siempre en la Catedral valenciana, en el Colegio Seminario San Pío V, en Requena y Sagunto (González Tornel 2004, 123-124; Pingarrón 1998, 120, 291-301, 503-508).

2. Francisco Padilla, tras Juan Pérez Castiel el arquitecto más solicitado en la Valencia del fin del siglo XVII, fue activo en la capital en la Capilla de la Comunión de la Iglesia de San Esteban, en la remodelación de la iglesia del convento de Santo Domingo, en la construcción de la iglesia del convento de Belén y de la iglesia del convento del Remedio, en el Oratorio de San Felipe Neri, en el claustro del 
ha sido considerada secundaria respecto a la de Pérez Castiel. Sin embargo, su producción arquitectónica demuestra las primeras aplicaciones de las teorías geométricas que tendrán gran fortuna en Valencia durante la primera mitad del siglo XVIII. Quizá por primera vez en el medio valenciano, un arquitecto se alejó de la propia práctica artesanal y gremial de su profesión hasta llegar a categorías más científicas (González Tornel 2004, 121-148; González Tornel 2005a, 44-48).

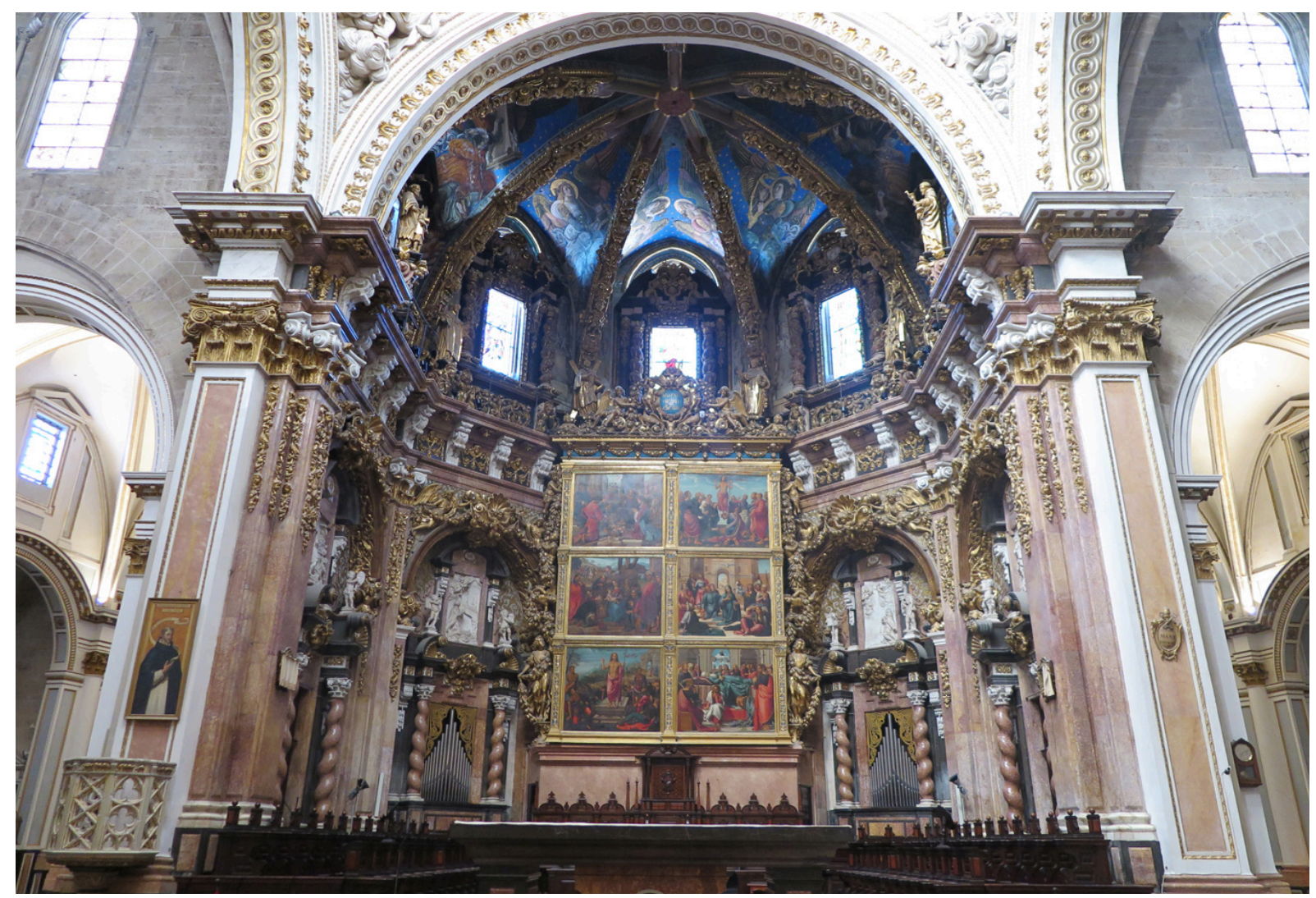

Figura 1. Juan Pérez Castiel, Catedral de Valencia (presbiterio), 1674-1682

En este panorama entre tradición e innovación, hay que destacar también el papel de algunos retableros, como los hermanos José y Domingo Cuevas y Leonardo Julio Capuz. Introduciendo el uso de materiales pétreos y alguna vez interfiriendo en las propias obras de cantería, contribuyeron a la entrada de elementos novedosos y de ruptura de la tradición gremial dominante aún en la ciudad (Ferrer Orts 1996, 55-63;

convento del Pilar y en la fachada de la Seo valenciana. Fuera de la capital, obras suyas se hallan en Meliana, Benaguacil, Albalat de la Ribera, Alboraya, etc. (González Tornel 2004, 124-148). 
González Tornel 2005a, 55-65). ${ }^{3}$ Las obras de todos estos artistas brevemente nombrados, si bien por un lado representaron una primera inversión de tendencia respecto a la tradición tardo-manierista vigente en la ciudad, manifestaban, no obstante, unas características aun fuertemente dependientes de los cansados modelos del pasado. En particular se distingue el uso de elementos decorativos repetitivos, incluso seriados: esa era la característica de las llamadas hojarascas que cubrieron los espacios sagrados renovados por los citados arquitectos. La finalidad de estas decoraciones era casi exclusivamente estética, es decir que estos primeros experimentos de barroco decorativo valenciano no tenían intentos iconográficos, sino que servían solo para sorprender al espectador, dejarlo sin aliento ante su riqueza y majestuosidad. El papel de trasmitir los mensajes contrarreformistas y evangélicos se le confiaba, más tradicionalmente, a pinturas y esculturas. Como se verá, todo ello cambió entre finales del siglo XVII y el siguiente, con la renovación barroca del templo de los Santos Juanes, donde realmente los adornos, en particular el estuco, comenzaron a desempeñar una finalidad iconográfica, aparte de ornamental, en íntima relación con pinturas y esculturas.

\section{El punto de inflexión: la llegada en Valencia de tres artistas foráneos}

La situación cambió radicalmente con la llegada a la ciudad de tres artistas foráneos cuya obra representó en Valencia el primer paso hacia un verdadero protagonismo del ornamento en la evolución del Barroco. El primero de ellos fue Antonio Aliprandi (16541718, en Valencia entre 1700 y 1705). Lombardo de origen, se había formado en Viena, trabajando en las habitaciones imperiales, donde tuvo ocasión de conocer al archiduque Carlos de Austria, aspirante al trono de España y junto al cual probablemente llegó a Valencia. Sus innovadoras intervenciones en la ciudad y su entorno, determinaron la desintegración entre producción arquitectónica y ornamento al contratar el lombardo directamente sus acciones decorativas basándose en sus propios diseños. ${ }^{4}$ Además, por

3. Para completar esta panorámica sobre la introducción del barroco decorativo en Valencia, se debe también tener en cuenta la injerencia en el medio artístico de la ciudad del grupo de los novatores. Se trataba de intelectuales relacionados con el mundo de las matemáticas como el jesuita José Zaragozá, Félix Falcó de Belaochaga, José Vicente del Olmo, Baltasar İñigo, Juan Bautista Corachán y el más famoso Tomás Vicente Tosca (González Tornel 2005a, 67-88). Junto con ellos aparecían de manera constante en las resoluciones artísticas y en la definición de los proyectos arquitectónicos de la ciudad también los canónigos Vicente Vitoria (1650-1709) y Antonio Pontons (noticias entre 1683 y 1706), ambos fuertemente vinculados artística y culturalmente a Italia (De Orellana 1967, 269-281; González Tornel 2005a, 89-103).

4. En Valencia, Antonio Aliprandi se dedicó principalmente al revestimiento con estuco de espacios sagrados antiguos, como la Iglesia de los Santos Juanes, en donde trabajó junto al escultor 
primera vez, un artífice se define a sí mismo como estucador, profesión que no estaba incluida dentro de ninguno de los tradicionales gremios y que, por lo tanto, pudo escapar a su rígido control. En consecuencia, las obras de Aliprandi supusieron en Valencia las primeras injerencias en el hecho arquitectónico de un artista ajeno a la práctica de la arquitectura. Estilísticamente, sus decoraciones hacen patente un atento estudio del natural, puesto que cada elemento floral y vegetal que él empleó puede relacionarse con una específica tipología vegetal (Bérchez 1982, 48-53; De Orellana 1967, 251, 586, 588; González Tornel 2002, 127-145; González Tornel 2005a, 106-198).

También de procedencia lombarda, Giovanni Giacomo Bertesi (1643-1710) se formó en su tierra como escultor. Al llegar a Valencia, se definió a sí mismo como arquitecto en el único documento en el cual hay constancia de él, fechado en 1702 (González Tornel 2005a, 227). De las obras que De Orellana le atribuyó, solo se conserva la grandiosa renovación barroca del templo de los Santos Juanes en Valencia (De Orellana 1967, 251). ${ }^{5}$ Su programa, a la vez iconográfico y decorativo, fue realizado enteramente en estuco y está basado en la representación de Jacob y sus hijos en las estatuas exentas entre los contrafuertes que separan las capillas y las alegorías reclinadas de dos en dos sobre los arcos de embocadura de las mismas (figura 2). Esta composición de los paños murales rompió completamente con los interiores que se habían hecho en Valencia hasta entonces y conecta con ejemplos contemporáneos italianos de ámbito romano y florentino (Bérchez 1982, 48-53; Gil Gay 1909, 20-45; González Tornel 2005a, 198-254). ${ }^{6}$

El tercer y último artista que llegó a Valencia en los años que rodearon el paso de siglo fue Conrad Rudolph, también relacionado, como Aliprandi, con el archiduque Carlos de Austria. Su nombre estuvo vinculado sobre todo con el concurso de 1701 para la realización de la fachada de la Catedral valenciana. Rudolph, de hecho, lo ganó y, frente a los artistas autóctonos cuyos proyectos fueron rechazados y entre los cuales destacaba el de Pérez Castiel, defendió su labor declarando que se había formado estudiando la técnica

Giacomo Bertesi, y la Capilla de San Pedro en la Seo de la ciudad. Además, decoró la Capilla de la Purísima en la Iglesia de la Compañía de Jesús de la ciudad y el Huerto de Pontons. Finalmente, desde 1705 es documentado en Cocentaina, donde realizó la que quizá pueda considerarse su obra maestra: los adornos y retablo de la Iglesia del Convento de la Virgen del Milagro en Cocentaina (González Tornel 2005a, 130-196).

5. Marcos Antonio de Orellana, el mayor biógrafo de los artistas valencianos de los siglos XVII y XVIII atribuyó a Giacomo Bertesi, además de la citada renovación de la Iglesia de los Santos Juanes, las decoraciones del salón del Huerto de Pontons y de la Capilla de la Concepción en la Iglesia de la Compañía de Jesús, ambas desaparecidas. Sin embargo, en las cartas de pago relativas a esta segunda obra, el nombre de Bertesi no figura, mientras que la dirección de las obras está ocupada por el estucador Antonio Aliprandi (González Tornel 2005a, 179-180, 236; Archivo del Reino

6. En particular, la relación entre estatuas alegóricas sentadas sobre los arcos de embocadura de las capillas y óvalos pictóricos era presente en la iglesia de Santa Maria in Vallicella en Roma según proyecto de Pietro da Cortona y en la Iglesia de la Annunziata de Florencia (González Tornel 2008, 127-128). 
de los antiguos y modernos arquitectos romanos. Precisamente Conrad Rudolph, llamado el Romano, introdujo en la ciudad el uso de la geometría cóncavo-convexa, sin precedentes en España y vinculada a las obras de Francesco Borromini y Guarino Guarini (De Orellana 1967, 215; González Tornel 2005a, 255-302; Pingarrón 1998, 130-142).

\section{La evolución de la profesión del ornamentista en el siglo XVIII}

\section{La eclosión del adorno}

Las innovaciones introducidas en el medio artístico valenciano por Aliprandi, Bertesi y Rudolph pronto fueron recibidas e introducidas en sus obras por los artistas autóctonos. Se trataba de personalidades que se habían formado en la tradición gremial y habían vivido de cerca la desintegración de los rígidos esquemas corporativos, debido al éxito en tierras valencianas de nuevos rasgos estilísticos procedentes de Italia y Europa central. El primero de estos artistas fue Francisco Vergara el Mayor (1681-1753), al cual se le confió la edificación de la fachada de la Seo valenciana después de que Rudolph abandonara Valencia. La influencia del proyecto rudolphiano será patente en todas las obras posteriores de Vergara, en particular en la renovación decorativa de la Iglesia de San Martín, cuyo friso presenta los trofeos eclesiásticos que Rudolph había previsto para los frisos de las cornisas de la fachada catedralicia (figura 3. González Tornel 2005a, 311-321; González Tornel 2005b, 41-51; Pingarrón 1997, 327-363; Pingarrón 1998, 141-142, 259-262). ${ }^{7}$

Hijo de Francisco Vergara, el escultor Ignacio Vergara (1715-1776) es conocido sobre todo por sus obras escultóricas, entre las cuales destacó el magnífico portal de piedra alabastrina del Palacio del Marqués de Dos Aguas, hoy en día sede del Museo Nacional de Cerámica González Martí. Realizó también proyectos arquitectónicos, como el de la portada lateral de la citada Iglesia de San Martín, y fue gran enseñante, antes en su taller privado y en seguida como profesor de escultura de la Academia de San Carlos, de la cual fue promotor junto con su hermano, el pintor José Vergara (Bérchez 1997, 16-107, 111-121; Pingarrón 1990, 67-82). Su producción en estuco es incierta y todavía por investigar. Es cierto que decoró las pechinas de la cúpula del

7. La labor de Francisco Vergara en la fachada de la Catedral y en San Martín fue fundamental incluso para artistas de la generación siguiente como Felipe Rubio, que insertó los trofeos eclesiásticos en su remodelación barroca de la Iglesia de Santa Catalina (González Tornel 2005a, 317-318; Pingarrón 1997, 334-338). 
escalón del citado Palacio de Dos Aguas, las cuales estilísticamente pueden vincularse también con la contrafachada y otros elementos decorativos de la cercana Iglesia de San Andrés (figura 4. Giannotta 2019, 223-225; González Tornel 2011, 102).

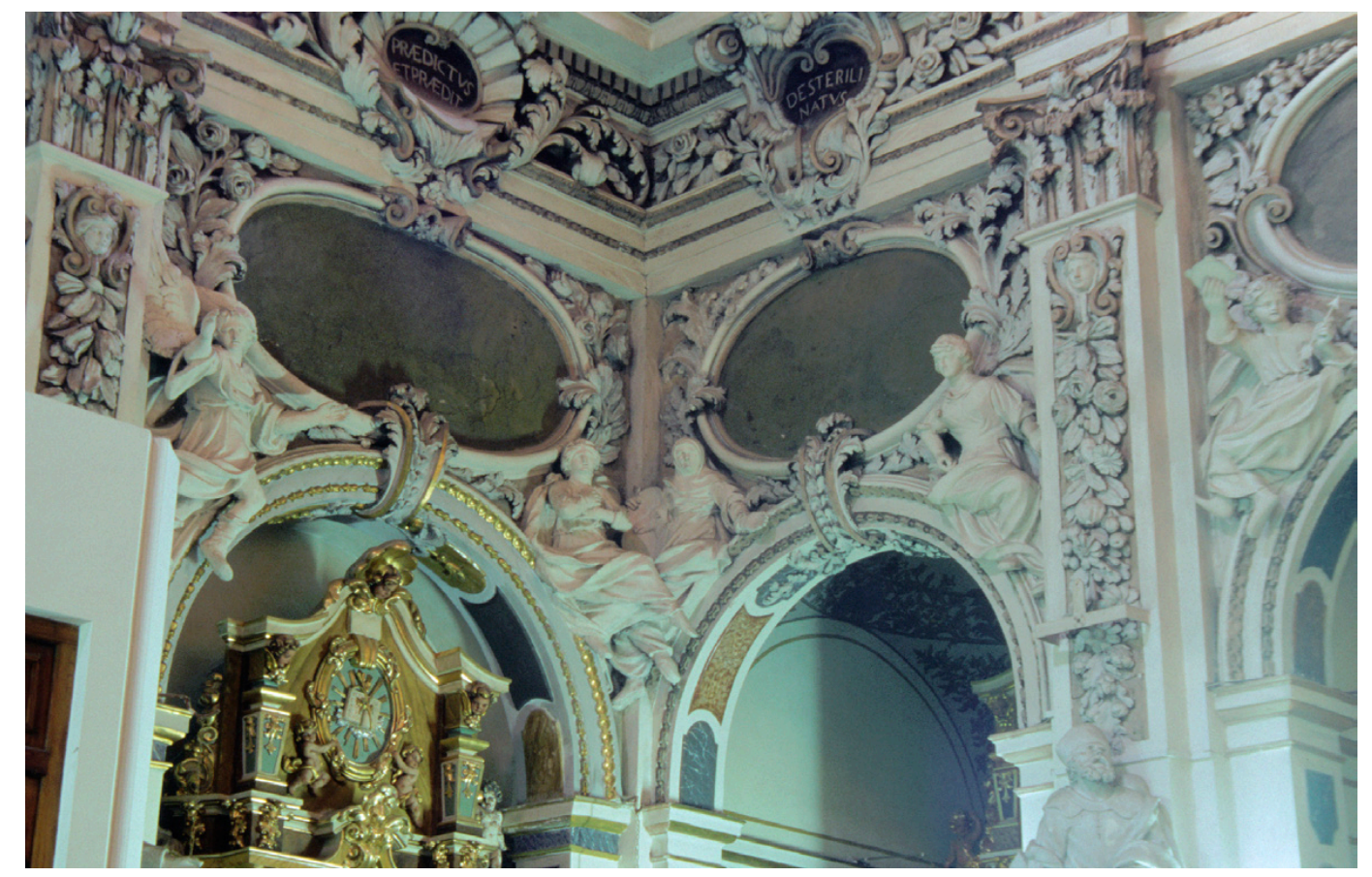

Figura 2. Giacomo Bertesi y Antonio Aliprandi, Iglesia de los Santos Juanes, Valencia, 1693-1702

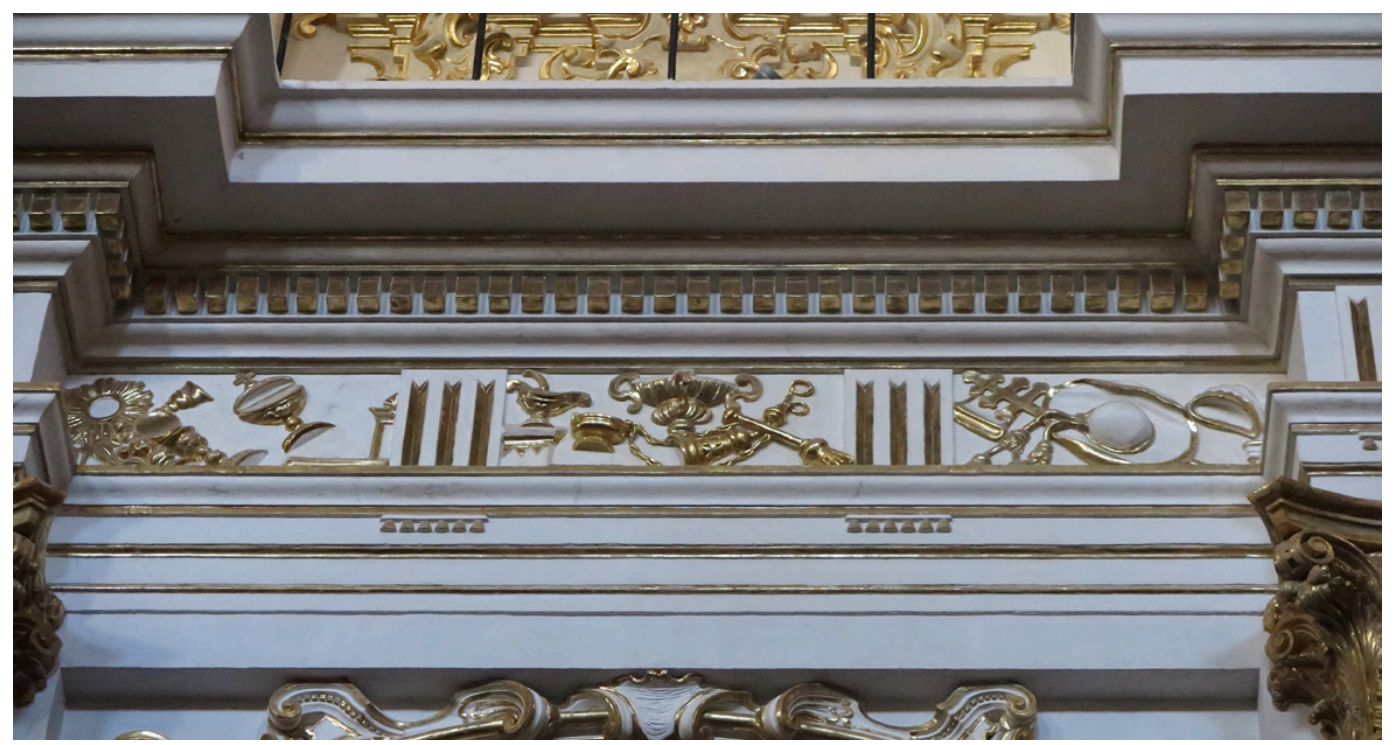

Figura 3. Francisco Vergara y José Herrero, Iglesia de San Martín Obispo y San Antonio Abad, Valencia, 1735-1755 
Otro estucador poco conocido y cuya obra merece analizarse en profundidad es el alicantino José Artigues (†1733). Fue activo durante el primer tercio del siglo XVIII sobre todo en la ciudad de Elche, donde, dentro del ámbito de edificación de la basílica de Santa María, talló su retablo, decoró las pechinas de su cúpula y proyectó su Puerta del Sol. Todas manifiestan una fuerte vinculación con las innovaciones de principios del siglo, sobre todo con la fachada de la Catedral valenciana de Rudolph y las intervenciones de Aliprandi en Cocentaina (Cañestro Donoso 2015, 192-196; González Tornel 2005a, 322323). Está todavía por documentar la participación de Artigues en la decoración de las capillas de San Antonio de Padua y de Santa Ana y la Virgen Niña que se encuentran enfrentadas en el Convento de San José, siempre en Elche. Realizadas respectivamente en 1724 y 1729, su decoración fue elaborada casi totalmente con el estuco que dialoga iconográficamente con las pinturas murales. El repertorio de conchas rugosas, estilizaciones vegetales y flores recuerdan precisamente a la citada obra de Artigues para la basílica de Santa María y, en última instancia, a las innovaciones de Aliprandi. En el estado actual de los estudios, esa atribución es incierta y la obra sigue siendo anónima (González Tornel 2005a, 323-325).

Otra obra anónima hasta la fecha que reclama un estudio profundo para dilucidar su autoría, fuentes e impacto, es la decoración del trasagrario, camarín y pasillo de la Iglesia del Convento de la Virgen del Niño Perdido en Caudiel (figura 5). Las obras para estos espacios empezaron en 1701 y tuvieron que ser concluidas en el año 1717. El trasagrario es el lugar en donde el adorno de estuco tiene más protagonismo llegando a una verdadera asfixia decorativa: conchas rugosas, hojas y flores carnosas, rocallas, caras de mancebos y putti rellenan totalmente las paredes hasta el interior de la cúpula. También en este caso es presente la relación entre los estucos y las pinturas, atribuidas a Gaspar de la Huerta (González Tornel 2005a, 322-332; Vilaplana 1987, 57-63). 


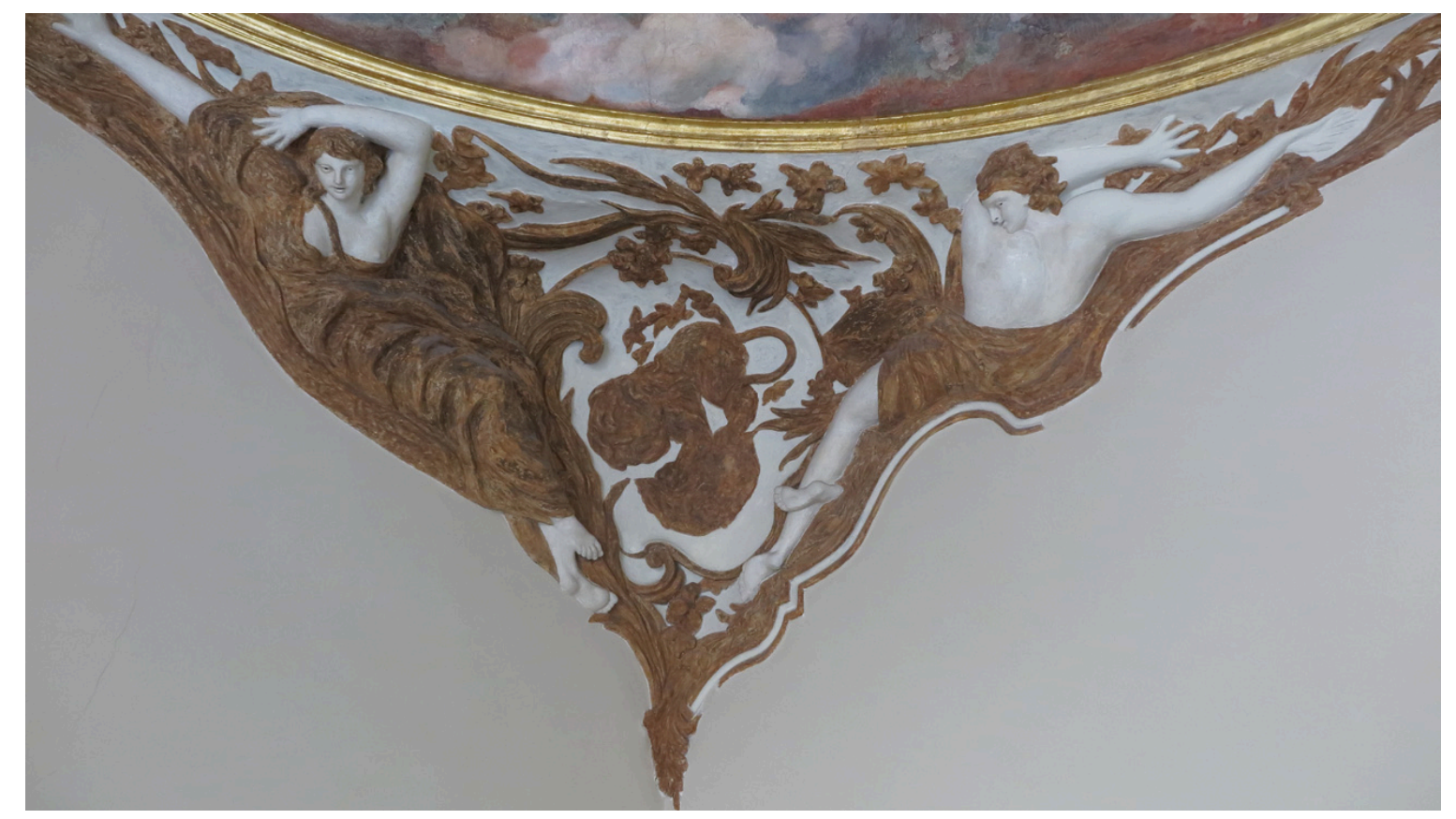

Figura 4. Ignacio Vergara, Palacio del Marqués de Dos Aguas (pechinas de la cúpula del antiguo escalón de honor), mitad del siglo XVIII

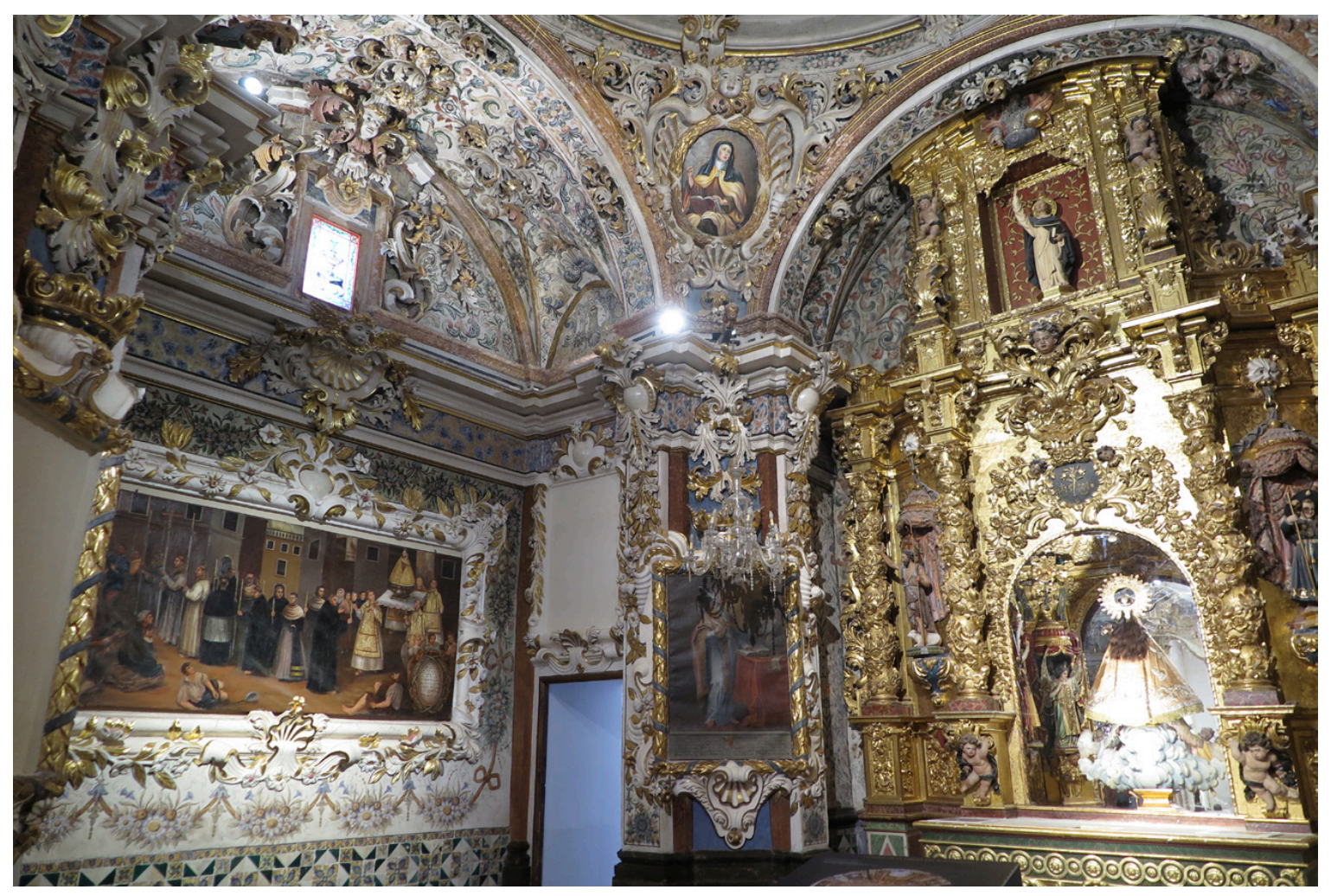

Figura 5. Anónimo, Santuario de la Virgen del Niño Perdido (trasagrario), Caudiel, 1701-1717 
El último de los artistas cuya obra espera una mayor profundización es el escultor y estucador Luis Domingo (1718-1767). Ejemplificó, mejor que ningún otro en Valencia, la personalidad del artista rococó, cuya obra difícilmente se puede encasillar en uno de los tradicionales ejercicios profesionales, siendo a la vez pintor, escultor, retablero, adornista y tallista. Además, debería aclararse su actividad como ceramista y proyectista de cerámicas, puesto que firmó las del refectorio del Convento de Santo Domingo en Orihuela. En consecuencia, puede considerarse como quien puso verdadero fin a la tradición gremial valenciana y, además, como el último verdadero ornamentista barroco antes de la implantación del academicismo. Tradicionalmente la obra maestra que se le atribuye es la renovación decorativa de la Iglesia de San Andrés. Sin embargo, las investigaciones archivísticas realizadas hasta la fecha solo han permitido aclarar que la obra de renovación empezó en 1751 y se limitó inicialmente al presbiterio y a los primeros dos tramos adyacentes de la nave. En 1753 se contrató a Félix Lorente la doradura de los estucos que entonces comprendían también el tercer tramo. El último dato documental es el libramiento a Bautista Pontons y Juan Simarro de ocho pedestales y medio, cuya obra se les pagó en $1765 .{ }^{8}$ La intervención en San Andrés constituyó el clímax de la ornamentación en estuco cuyo desarrollo hasta aquí se ha analizado. En particular, el estuco tuvo en ella una fundamental finalidad iconográfica, junto con las pinturas y las cerámicas (figura 6). Por lo tanto, es necesario aclarar el papel de Domingo u otros artistas en esta intervención y devolver a la Iglesia de San Andrés la importancia que tiene en el ámbito artístico valenciano de entre el barroco y el neoclasicismo.

8. Archivo del Reino de Valencia, Protocolos notariales, signaturas 4995-5012, años 1751-1768. Notario: Agustín Bonet. 


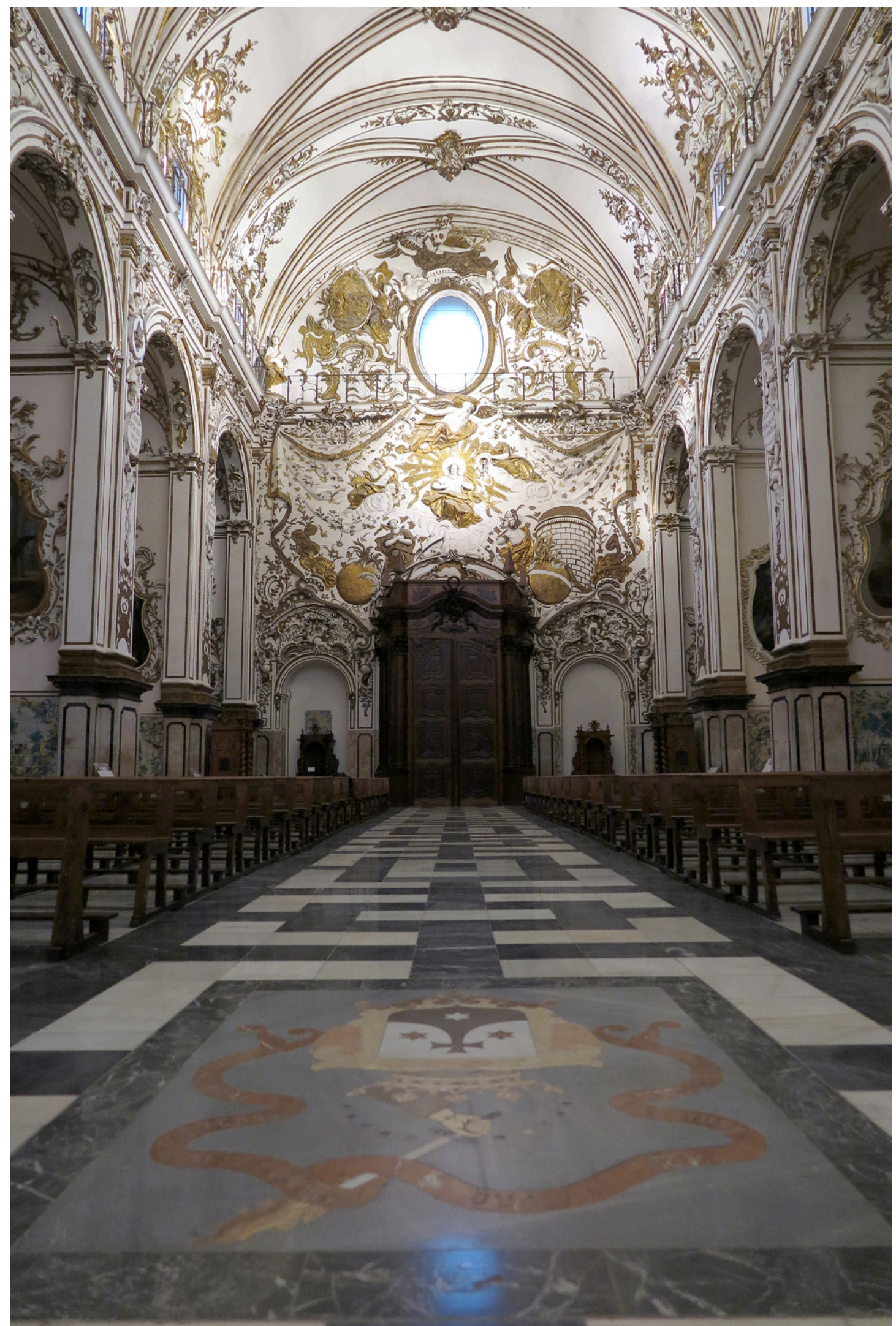

Figura 6. Félix Lorente y otros, Iglesia de San Andrés, Valencia, post 1751 
Hacia una común matriz europea de los estucos dieciochescos valencianos

El ornamento en estuco como base de los revestimientos arquitectónicos fue hiperdesarrollado en toda Europa a lo largo del siglo XVIII. La razón de todo ello se basa en la dispersión en todo el continente de los estucadores lombardos y del Valle Intelvi, que en su tierra se habían formado en una técnica de tradición centenaria. De ahí venían también Giacomo Bertesi y Antonio Aliprandi, a los cuales se debió, como he referido, la introducción de elementos estilísticos y procedimientos técnicos totalmente novedosos en el medio valenciano (González Tornel 2005a, 106-112). Además, la aportación cultural de toda una élite de canónigos y aficionados vinculada a Italia y en particular a Roma hizo que en los grandiosos programas decorativos realizados en Valencia se introdujesen los postulados estilísticos de Bernini, Borromini, Pietro da Cortona, el Baciccia y Luca Giordano. Mientras tanto lo mismo ocurría en el resto de Europa. Por ejemplo, en los mismos años en que en Valencia estucaban Aliprandi o Luis Domingo, en Palermo, Sicilia, Giacomo Serpotta (1656-1732) y su taller revestían oratorios e iglesias con parecidas decoraciones en estuco empleando los mismos rasgos estilísticos citados e iguales técnicas ejecutivas.

La creación de la Real Academia y el fin del desarrollo de la profesión de adornista

En 14 de febrero de 1768 surgió en Valencia la Real Academia de Bellas Artes de San Carlos. Habían fomentado su creación algunos de los artistas que se habían formado en la primera mitad del siglo, cuando los tradicionales esquemas gremiales daban el paso a las innovaciones introducidas en Valencia por artistas extranjeros. Entre ellos destacaban Ignacio Vergara y Luis Domingo, que, como se ha considerado, se habían comprometido con la decoración barroca de derivación italiana y centroeuropea. Mientras tanto, una nueva generación de artistas estaba naciendo y estaba vinculada con nuevos principios estilísticos de carácter neoclásico. Entre ellos estuvo Vicente Gascó (1734-1802), que puede considerarse el verdadero artífice de la reforma en sentido neoclásico e ilustrado de la arquitectura en Valencia. Su actividad de enseñanza en la sección de arquitectura de la Academia y sus proyectos para algunos de los edificios más emblemáticos de la ciudad se convirtieron en una verdadera arma contra el decorativismo asfixiante de las producciones anteriores, reputados ejemplos del imperante mal gusto que el academicismo se proponía 
cancelar. ${ }^{9}$ A raiz del surgimiento de la Academia de San Carlos se puede considerar concluido el proceso de evolución del adorno barroco brevemente descrito.

\section{Conclusiones}

En conclusión, el estudio de manera independiente y específica de los adornistas y de la producción artística en estuco en Valencia a lo largo del siglo XVIII, inexistente hasta la actualidad, tiene un considerable impacto académico. Dicho impacto será positivo en la medida en que ponga de manifiesto artistas poco conocidos hasta la fecha, a la aclaración de técnicas y modelos inusuales en la Valencia gremial, al desvelamiento de programas iconográficos aún ocultos y, sobre todo, a la conexión de la ciudad con un contexto cultural europeo. En última instancia se pretende que el mayor conocimiento de las obras y artistas estudiados suponga una revalorización de los mismos que contribuya a la conservación y transmisión del patrimonio cultural valenciano estudiado.

\section{Bibliografía}

Aldana Fernández, Salvador. 1968. «El arquitecto barroco Juan Pérez Castiel». Boletín de la Sociedad Castellonense de Cultura 43: 55-87.

Bérchez, Joaquín. 1993. Arquitectura barroca valenciana. Valencia: Bancaja.

Bérchez, Joaquín. 2009. «Ideario ilustrado y académico valenciano en la renovación de la Catedral de Segorbe». La Real Academia de Bellas Artes de San Carlos en la Valencia ilustrada, editado por Romà de la Calle, 187-192. Valencia: Universitat de València.

Cañestro Donoso, Alejandro. 2015. Arquitectura y programas artísticos en la provincia de Alicante durante la Edad Moderna. Madrid: CSIC.

Ferrer Orts, Albert. 1996. «A propósito de tres retablos valencianos». Archivo de Arte Valenciano 77: 55-63.

9. Entre sus obras se citan el proyecto irrealizado para la Capilla de San Vicente Ferrer en el convento de Santo Domingo, la Capilla de la Comunión de la Iglesia de los Desamparados, el proyecto de ampliación de la Iglesia de Càlig, la decoración de la Capilla de la Comunión de la Iglesia Mayor de Vila-real, solo por citar algunos ejemplos (Bérchez 2009, 187-192). 
Giannotta, Gaetano. 2019. «The Church of Saint Andrew of Valencia. State of the issue and research's objectives». En 11th European Symposium on Religious Art, Restoration \& Conservation. Proceedings Book, editado por María Luisa Vázquez de Ágredos-Pascual (et al.), 223-225. Torino: Kermes Books.

Gil Gay, Juan. 1909. Monografia histórico-descriptiva de la Real parroquia de los Santos Juanes de Valencia. Valencia: Tipografía de San José de J. Canales.

González Tornel, Pablo. 2002. «Antonio Aliprandi, un estucador lombardo en la Valencia de 1700». Espacio, tiempo y forma. Serie VII, Historia del Arte 15: 127-145.

González Tornel, Pablo. 2004. «El arquitecto barroco Francisco Padilla. Una visión de la arquitectura desde la geometría y la tratadística». Espacio, tiempo y forma. Serie VII, Historia del Arte 17: 121-148.

González Tornel, Pablo. 2005a. Arte y arquitectura en la Valencia de 1700. Valencia: Institució Alfons el Magnànim.

González Tornel, Pablo. 2005b. «Francisco Vergara y los revestimientos arquitectónicos del barroco valenciano en las primeras décadas del siglo XVIII». Archivo de Arte Valenciano 86: 41-51.

González Tornel, Pablo. 2008. «Barroquizar la arquitectura. Intervenciones de signo barroco en construcciones eclesiásticas medievales de la ciudad de Valencia». En Historia de la ciudad V. Tradición y progreso, 131-152. Valencia: Colegio Oficial de Arquitectos de la Comunidad Valenciana.

González Tornel, Pablo. 2011. «El ornamento arquitectónico como base del cambio de gusto en la Valencia de mediados del siglo XVIII. De los estucos de la Parroquia de San Andrés a los modelos académicos de Vicente Gascó en la Capilla del Carmen». Ars Longa 20: 97-108.

Orellana, Marcos Antonio de. 1967. Biografía pictórica valentina o vida de los pintores, arquitectos, escultores y grabadores valencianos. Valencia: Ayuntamiento de Valencia. Pingarrón, Fernando. 1990. «Portadas de la iglesia parroquial de San Martín de Valencia». Archivo de Arte Valenciano 71: 67-82.

Pingarrón, Fernando. 1997. «Algunos documentos sobre las reformas tardobarrocas de las iglesias de San Andrés, Santa Catalina y San Martín en Valencia a mediados del siglo XVIII». Saitabi 47: 327-363.

Pingarrón, Fernando. 1998. Arquitectura religiosa del siglo XVII en Valencia, València: Ayuntamiento de Valencia.

Vilaplana Zurita, David. 1987. «Una obra maestra del Barroco valenciano: el santuario de la Virgen del Niño Perdido en Caudiel». Archivo de Arte Valenciano 68: 57-63. 


\title{
Estereotipos de género, elección de estudios universitarios y práctica deportiva competitiva en una muestra de deportistas CADU de la Universitat Jaume $I^{1}$
}

\author{
Jahel Molina Ulldemolins, \\ M. Carmen Pastor Verchili, María Escrig Mateu, \\ Carlos Hernando Domingo, Sonia Reverter Bañón \\ jahel.molina@uji.es
}

Actualmente las mujeres equiparan o superan el número de hombres que completan el nivel de educación superior, lo que sugiere una igualdad entre sexos. Sin embargo, siguen eligiendo titulaciones universitarias distintas, con una menor participación de mujeres en áreas académicas consideradas tradicionalmente masculinas. Los estereotipos de género también influyen en la práctica deportiva competitiva, puesto que las mujeres que se identifican con rasgos más masculinos presentan una menor tasa de abandono. La influencia de estereotipos de género en el deporte y la elección de estudios universitarios se deben parcialmente a la interiorización de la identidad de género, definida por las expectativas de la persona y del entorno sociocultural. Nuestro estudio pretende explorar la relación entre estereotipos de género y elección de titulación universitaria en una muestra de 125 estudiantes de la Universitat Jaume I (61 mujeres), con una media de edad de 20 años, que competían en diferentes deportes de la liga universitaria. Se administró una batería de 
cuestionarios en formato de lápiz y papel, entre ellos la escala de Estereotipos de Género Actuales (EGA) y el Cuestionario de Datos Sociodemográficos. Los análisis preliminares sugieren una perpetuación del patrón tradicional de elección de titulación, con un desequilibrio entre sexos en todos los centros, a excepción de la Facultad de Ciencias Jurídicas y Económicas. Sin embargo, no se observaron diferencias significativas en las puntuaciones en el EGA, lo que no confirma la hipótesis de que los estudiantes que practican deporte de forma competitiva tendrían un perfil más masculinizado. Nuestros resultados sugieren que los estereotipos de género afectarían únicamente a las expectativas del entorno sociocultural para cada sexo, por influencia directa de los roles de género. Esto explicaría que hombres y mujeres sigan eligiendo estudios típicamente masculinos o femeninos, independientemente de que practiquen deporte de competición. Futuros estudios deberían incorporar un grupo control equiparado en características sociodemográficas que no haya practicado deporte competitivo.

Palabras clave: estereotipos de género, roles de género, práctica deportiva competitiva, elección de estudios universitarios, población universitaria

\section{Introducción}

Los estereotipos son aquellas creencias populares sobre los atributos que caracterizan a un grupo social y sobre el que hay un acuerdo básico (Mackie 1973, 431-447). No reflejan una imagen exacta de la sociedad; más bien, el rol que desempeñan las personas dentro de los grupos sociales. La función principal es darnos un valor funcional y adaptativo que nos permita entender el mundo de forma simple y predecir futuros acontecimientos. Además, en la socialización, nos facilitan la identidad social y la conciencia de pertenecer a un grupo (González Gavaldón 1999). Los estereotipos surgen del medio social y se aprenden a través de los procesos de socialización, especialmente durante la adolescencia e infancia, y se afianzan en la edad adulta. 
Uno de los estereotipos que más se ha mantenido a lo largo de la historia ha sido el asociado al género, que se ha generalizado en la mayoría de contextos sociales y ha derivado en una creencia uniforme de lo que se considera ser mujer y hombre en casi todo el mundo. En la cultura occidental, los roles que tradicionalmente se han asignado a los hombres han sido orientados al trabajo y la racionalidad, formando el estereotipo masculino. En cambio, las cualidades de cuidadora, calidez y sensibilidad se han asociado al estereotipo femenino (González Gavaldón 1999). Una vez las personas han interiorizado los roles de género, tanto hombres como mujeres tienden a actuar de forma congruente con ellos, convencidos de que los hombres están orientados hacia el trabajo y las mujeres hacia las relaciones interpersonales por naturaleza, sin ser conscientes de que se debe a una interiorización de los estereotipos y roles de género que se han mantenido generación tras generación durante los procesos de socialización.

Esta interiorización de los estereotipos y roles de género, junto con un contexto también estereotipado sobre lo que se espera de un hombre y una mujer, son los encargados de perpetuar un patrón en la elección de carrera de las y los jóvenes, que les impulsa a elegir materias de estudio diferentes, acorde con su rol de género. Si observamos las estadísticas de la mayoría de las universidades españolas, podremos observar que las mujeres eligen mayoritariamente estudios relacionados con actividades de atención y cuidados, mientras que los hombres prefieren cursar grados relacionados con el sector tecnológico e industrial. Observando los datos de la Universitat Jaume I (Unitat d'Igualtat UJ 2018), podemos observar un desequilibrio entre sexos en casi todos los centros, facultades y Escuela. En la Facultad de Ciencias Humanas y Sociales (FCHS), las mujeres representan el 70,83 \% y los hombres el 29,17\%, con un desequilibrio por sexos con una diferencia de 41,65 \% a favor de las mujeres. En la Facultad de Ciencias Jurídicas y Económicas (FCJE), las mujeres representan el 56,26 \% y los hombres el $43,74 \%$, lo que la convierte en la única facultad que presenta un equilibrio por sexos con una diferencia de un 12,52 \% a favor de las mujeres. En cambio, en la Escuela Superior de Tecnología y Ciencias Experimentales (ESTCE), las mujeres representan el 26,60 \% y los hombres el 73,40 \%, lo que muestra un desequilibrio entre sexos con una diferencia de $46,80 \%$ a favor de los hombres. Finalmente, en la Facultad de Ciencias de la Salud (FCS), las mujeres representan el 73,90 \% y los hombres el 26,10\%, con un desequilibrio entre sexos del $47,80 \%$ a favor de las mujeres. Estos resultados son coincidentes con el resto de las universidades valencianas y españolas, donde la rama de ciencias jurídicas y económicas es la 
más equilibrada y la rama de ingenierías y arquitectura es la más desequilibrada, con una mayor participación de hombres.

Cuando hablamos del deporte competitivo, apenas se cuestionan las diferencias de género, ya que en algunos casos se perciben como resultado de factores biológicos naturales (Chalabaev et al. 2013, Clément-Guillotin et al. 2013), lo que soslaya tanto los factores ambientales como los estereotipos y roles de género que influyen en la participación y el rendimiento de la práctica deportiva competitiva. Pese a que la participación en la práctica deportiva competitiva de las mujeres está aumentando de forma notable en las últimas décadas, se sigue considerando que el área deportiva es mayoritariamente de dominio masculino (Riemer y Visio 2003). En la actualidad, se sigue hablando de deportes femeninos y masculinos. Esta clasificación por género de los deportes va a depender de las características de deporte o del tipo de actividad que se realiza, entendiendo como deportes masculinos aquellos que requieran fuerza, velocidad, que incluyen contacto físico, el cara a cara o la agresividad. En cuanto a los deportes neutrales, el análisis es inexacto, ya que va a depender de en qué cualidad del deporte nos centremos. En cambio, los deportes considerados más femeninos incluyen características como la expresividad, la gracia y la estética, características que se definen aparentemente como más femeninas (Hardin y Greer 2009). De nuevo, en esta diferenciación del deporte por género se observa, por una parte, la propia interiorización de los estereotipos y roles de género y, por otra, la influencia de las expectativas e ideales del entorno cultural de lo que se espera de un hombre y una mujer.

\section{Objetivos}

El presente estudio tiene como objetivo explorar los estereotipos de género en una muestra representativa de deportistas universitarias/os de la Universitat Jaume I (UJI). Se pretende investigar si la percepción de los estereotipos de género en personas que realizan práctica deportiva competitiva también influye en la elección de la carrera universitaria.

Para ello se han formulado diferentes hipótesis que permiten englobar los diferentes objetivos del trabajo. En primer lugar, se prevé que la elección de carrera de las y los deportistas estará más sesgada hacia carreras consideradas más masculinas, ya que el deporte se considera de dominio masculino. Por tanto, 
en las y los deportistas predominarán los estereotipos masculinos. En segundo lugar, se explorará si los hombres y mujeres puntúan alto en los estereotipos pertenecientes a su rol de género. En tercer lugar, se comprobará si los centros que están más masculinizados puntúan más alto en estereotipos de género masculinos y aquellos considerados más femeninos puntúan más alto en estereotipos de género femeninos. Es decir, que la Escuela Superior de Tecnología y Ciencias Experimentales puntúe más alto en estereotipos masculinos, mientras que la Facultad de Ciencias Humanas y Sociales, junto a la Facultad de Ciencias de la Salud, puntúen más alto en estereotipos femeninos. Y, por último, se prevé que la Facultad de Ciencias Jurídicas y Económicas obtenga una puntuación menos orientada hacia los polos.

\section{Participantes}

La muestra experimental fue de 125 estudiantes, de las cuales 61 eran mujeres, con una media de edad de 20 años $(\mathrm{DT}=2,89)$. Los y las participantes de la muestra realizaban deporte competitivo, ya que formaban parte de los diferentes equipos CADU que representan a la Universitat Jaume I en la liga universitaria. La muestra entrenaba una media de 6,30 horas a la semana $(\mathrm{DT}=3,88)$ y llevaba una media de 7,5 años compitiendo (DT $=4,71$ ).

\section{Instrumentos de evaluación}

Escala de Estereotipos de Género Actuales (EGA; Castillo-Mayén y MontesBerges 2007): mide estereotipos de género utilizados en la actualidad para describir a las personas en general, o a los hombres y mujeres en particular. Consta de 40 adjetivos con una respuesta tipo Likert de 5 puntos: $1=$ En absoluto característico de mí, 2 = Poco característico de mí, 3 = Ni característico ni no característico, $4=$ Bastante característico de mí y $5=$ Totalmente característico de mí. De los 40 adjetivos, 20 representan estereotipos femeninos, 10 definen cualidades positivas y 10 negativas. Un ejemplo de ítems sería: cálido/a y sumiso/a, respectivamente. Los otros 20 son adjetivos considerados estereotipos masculi- 
nos, formado por 10 positivos y 10 negativos; por ejemplo: con éxito profesional y maltratador/a respectivamente.

\section{Procedimiento}

El presente trabajo corresponde a una parte de los resultados preliminares obtenidos de una investigación más amplia acerca del funcionamiento psicológico en la práctica deportiva competitiva, en las ligas CADU con estudiantes universitarias/os de la Universitat Jaume I. Todas las y los participantes acudieron a la Universitat Jaume I para realizar sus desplazamientos en las diferentes competiciones CADU. Durante los desplazamientos a las diferentes competiciones se administró una batería de instrumentos psicológicos en formato papel, incluyendo la Escala de Estereotipos de Género Actuales (EGA). Además, se obtuvieron los datos sociodemográficos de las y los participantes (por ejemplo, titulación que estudian, género/sexo, el deporte que practicaban, edad, los años que llevan compitiendo y horas de entrenamiento semanales).

\section{Análisis estadísticos}

En primer lugar, se calcularon el número de participantes que realizaban práctica deportiva competitiva para cada centro, facultades y escuela, de la Universitat Jaume I. A continuación, se calcularon las medias y desviaciones típicas de las medidas de autoinforme utilizadas en el presente estudio, para el grupo experimental (hombres y mujeres; centro al que pertenece el grado que estudian; grupo de hombres y mujeres por cada centro al que pertenece el grado que estudian). En segundo lugar, se realizaron pruebas $t$ de Student para investigar si existían diferencias entre el grupo experimental (hombres y mujeres, comparación de los diferentes centros, comparación de los diferentes centros para el grupo de hombres y mujeres) para el instrumento de evaluación (EGA). Los análisis fueron realizados con el paquete estadístico IBM SPSS Statistics versión 21 y el software JMP 9.0.1 (SAS Institute Inc.). 


\section{Resultados y análisis}

En primer lugar, se calculó la distribución de participantes por centros de la Universitat Jaume I (diferentes facultades y escuela). Se obtuvo el número de mujeres y hombres que practicaban deporte de forma competitiva en cada centro (véase figura 1).

A continuación, se calcularon los estadísticos descriptivos (medias y D.T.) del EGA desagregados por sexo (véase tabla 1). Por otra parte, también se calcularon los estadísticos descriptivos (medias y D.T.) del EGA distribuidos por centros: ESTCE, FCJE, FCHS y FCS (véase tabla 2). Igualmente, se calcularon estos valores para el grupo de mujeres y hombres que practicaban deporte competitivo, para cada centro por separado (véase tabla 2).

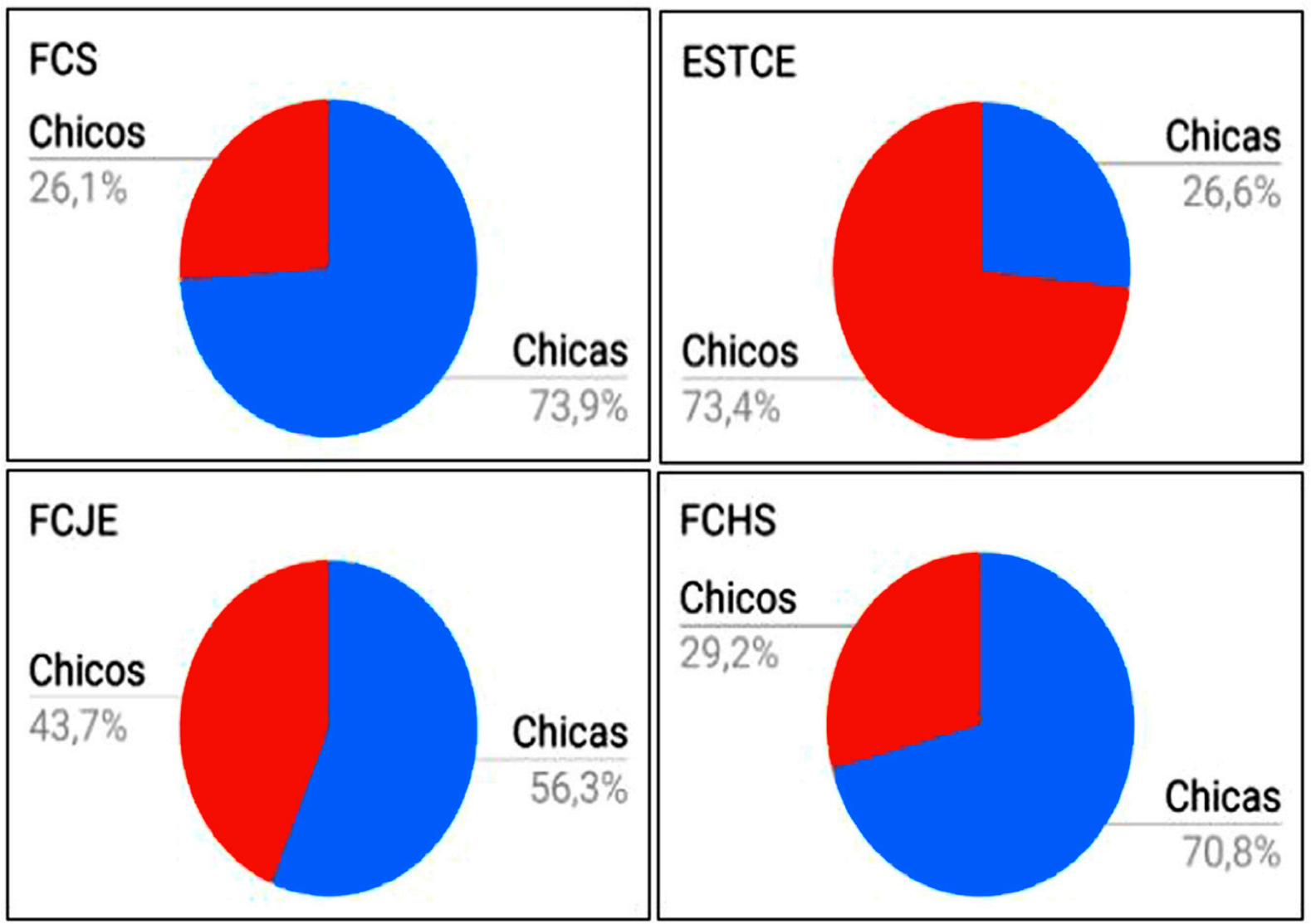

Figura 1. Distribución de participantes por género y por centros de la Universitat Jaume I 
Tabla 1. Medias (D.T.) de las puntuaciones en las subescalas del EGA, desagregadas por sexo. Comparaciones (pruebas $t$ de Student) entre chicas y chicos, para cada subescala por separado

\begin{tabular}{lcccccc} 
& \multicolumn{2}{l}{ Mujeres } & \multicolumn{2}{l}{ Hombres } & \multicolumn{2}{c}{ t de Student } \\
\hline & N & Media (D.T.) & N & Media (D.T.) & $\boldsymbol{t}$ & $\boldsymbol{p}$ \\
\hline EGA & & & & & & \\
Hombres Negativo & 61 & $17,39(3,41)$ & 64 & $18,92(4,57)$ & $-2,11$ &, $037^{*}$ \\
Hombres Positivo & 61 & $31,72(4,81)$ & 64 & $32,66(4,60)$ & $-1,11$ &, 269 \\
Mujeres Negativo & 61 & $23,05(4,97)$ & 64 & $22,28(5,07)$ & 0,85 &, 395 \\
Mujeres Positivo & 61 & $38,43(4,10)$ & 64 & $37,26(4,85)$ & 1,44 &, 152 \\
\hline
\end{tabular}

Tabla 2. Medias (D.T.) de las puntuaciones en las subescalas del EGA, desagregadas por sexo, para cada centro de la Universitat Jaume I (escuela y facultades) por separado

\begin{tabular}{llllllll} 
& ESTCE & & FCJE & & FCHS & \multicolumn{2}{l}{ FCS } \\
\hline N & M (D.T.) & N & M (D.T.) & N & M(D.T.) & N & M (D.T.) \\
\hline
\end{tabular}

\section{TOTAL}

Hombres Negativo $47 \quad 18,44(4,89) \quad 27 \quad 17,85(4,37) \quad 30 \quad 17,97(3,19) \quad 21 \quad 18,29(3,05)$

Hombres Positivo $47 \quad 34,23(4,41) \quad 27 \quad 30,22(4,25) \quad 30 \quad 30,13(4,32) \quad 21 \quad 33,14(4,43)$

$\begin{array}{lllllllll}\text { Mujeres Negativo } & 47 & 22,70(4,79) & 27 & 22,37(6,16) & 30 & 21,93(4,58) & 21 & 23,95(4,59)\end{array}$

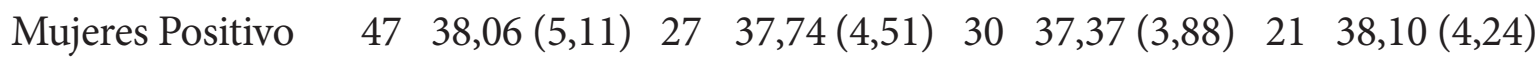

\section{CHICAS}

Hombres Negativo $15 \quad 16,87(4,37) \quad 15 \quad 17,00(2,85) \quad 16 \quad 16,94(2,98) \quad 15 \quad 18,80(3,21)$

Hombres Positivo $15 \quad 35,53(3,50) \quad 15 \quad 29,00(3,63) \quad 16 \quad 29,00(4,50) \quad 15 \quad 33,53(4,05)$

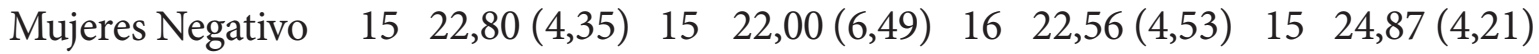

Mujeres Positivo $15 \quad 40,13(5,01) \quad 15 \quad 38,47(2,61) \quad 16 \quad 37,13(3,88) \quad 15 \quad 38,07(4,35)$ 


\begin{tabular}{llllllll} 
& ESTCE & & FCJE & & FCHS & \multicolumn{2}{l}{ FCS } \\
\hline $\mathrm{N}$ & M (D.T.) & $\mathrm{N}$ & $\mathrm{M}$ (D.T.) & $\mathrm{N}$ & $\mathrm{M}$ (D.T.) & $\mathrm{N}$ & $\mathrm{M}$ (D.T.) \\
\hline
\end{tabular}

\section{CHICOS}

Hombres Negativo $32 \quad 19,19(5,02) \quad 12 \quad 18,92(5,71) \quad 14 \quad 19,14(3,12) \quad 6 \quad 17,00(2,37)$

Hombres Positivo $32 \quad 33,63(4,72) \quad 12 \quad 31,75(4,61) \quad 14 \quad 31,43(3,86) \quad 6 \quad 32,17(5,53)$

$\begin{array}{lllllllll}\text { Mujeres Negativo } 32 & 22,66(5,05) & 12 & 22,83(5,97) & 14 & 21,21(4,69) & 6 & 21,67(5,09)\end{array}$

Mujeres Positivo $32 \quad 37,09(4,93) \quad 12 \quad 36,83(6,15) \quad 14 \quad 37,64(4,01) \quad 6 \quad 38,17(4,36)$

En cuanto a las pruebas $t$ de Student realizadas para comparar mujeres y hombres en cada subescala de EGA (véase tabla 1), los resultados mostraron diferencias de sexo significativas. Concretamente, en la subescala de Estereotipos Hombres Negativos, con una mayor puntuación del grupo de los hombres.

También se realizaron pruebas $t$ de Student comparando el grupo de hombres y mujeres, para cada centro por separado (véase tabla 3). Únicamente se observaron diferencias de sexo significativas para la ESTCE y la FCHS. Más concretamente, en la subescala de Estereotipos Mujeres Positivo para la ESTCE, con una mayor puntuación para las mujeres. En la FCHS, se observaron diferencias significativas en la subescala de Estereotipos Hombres Negativo, con una mayor puntuación para el grupo de los hombres. Para el resto de centros, no se encontraron diferencias significativas.

Además, se realizaron pruebas t de Student para comparar los diferentes centros (véase tabla 4). Observamos diferencias significativas en la comparación entre Centros en la ESTCE-FCJE, con la ESTCE-FCHS y la FCHS-FCS. Respecto a la comparación ESTCE-FCJE, observamos diferencias significativas en la subescala de Estereotipos Hombres Positivo del EGA, con una mayor puntuación para la ESTCE. En la comparación de la ESTCE-FCHS, se aprecian diferencias significativas en la subescala de Estereotipos Hombre Positivo del EGA con una mayor puntuación para la ESTCE. Finalmente, comparando la FCHS-FCS, podemos observar diferencias significativas para la subescala de Estereotipos Hombres Positivos del EGA, con una mayor puntuación para la FCS. 
Tabla 3. Comparaciones (pruebas $t$ de Student) entre chicas y chicos de cada centro, para cada subescala por separado del EGA

\begin{tabular}{lccccccccc} 
& \multicolumn{2}{c}{ ESTCE } & \multicolumn{2}{c}{ FCJE } & \multicolumn{2}{c}{ FCHS } & \multicolumn{2}{c}{ FCS } \\
\cline { 2 - 9 } & $\boldsymbol{t}$ & $\boldsymbol{p}$ & $\boldsymbol{t}$ & $\boldsymbol{p}$ & $\boldsymbol{t}$ & $\boldsymbol{p}$ & $\boldsymbol{t}$ & $\boldsymbol{p}$ \\
\hline EGA & & & & & & & & \\
Hombres Negativo & $-1,54$ &, 131 & $-1,14$ &, 266 & $-1,83$ &, $057^{*}$ & $-1,24$ &, 231 \\
Hombres Positivo & 1,39 &, 170 & $-1,69$ &, 106 & $-1,57$ &, 127 & 0,63 &, 537 \\
Mujeres Negativo & 0,10 &, 925 & $-0,34$ &, 734 & 0,80 &, 431 & 1,49 &, 153 \\
Mujeres Positivo & 1,96 &, $056^{*}$ & 0,86 &, 404 & $-0,36$ &, 722 & $-0,05$ &, 963 \\
\hline
\end{tabular}

Nota: Los valores en negrita son estadísticamente significativos $(p<, 05)$ en contrastes de una cola.

Posteriormente, se realizaron pruebas $t$ de Student comparando los centros desagregados por sexos (véase tabla 4). Comparando las chicas de los diferentes centros, se observan diferencias significativas entre los centros ESTCE-FCJE, ESTCE-FCHS, FCJE-FCS y FCHS-FCS. En la comparación de las chicas de la ESTCEFCJE, observamos diferencias significativas en la subescala de Estereotipo Hombre Positivo del EGA, con una mayor puntuación de las mujeres de la ESTCE. En la comparación de las mujeres ESTCE-FCHS, podemos observar diferencias significativas en la subescala de Estereotipo Hombre Positivo del EGA, con una mayor puntuación de las mujeres de la ESTCE. Tras comparar las mujeres de la FCJE-FCS, se encontraron diferencias significativas en la subescala de Estereotipo Hombre Positivo del EGA, con una mayor puntuación de las mujeres de la FCS. Comparando las mujeres de la FCHS-FCS, se obtuvieron diferencias significativas para la subescala de Estereotipo Hombre Positivo, con una mayor puntuación de las mujeres de la FCS. Finalmente, en la comparación de los hombres de los diferentes centros, no se observaron diferencias significativas en ninguna de las comparaciones. 


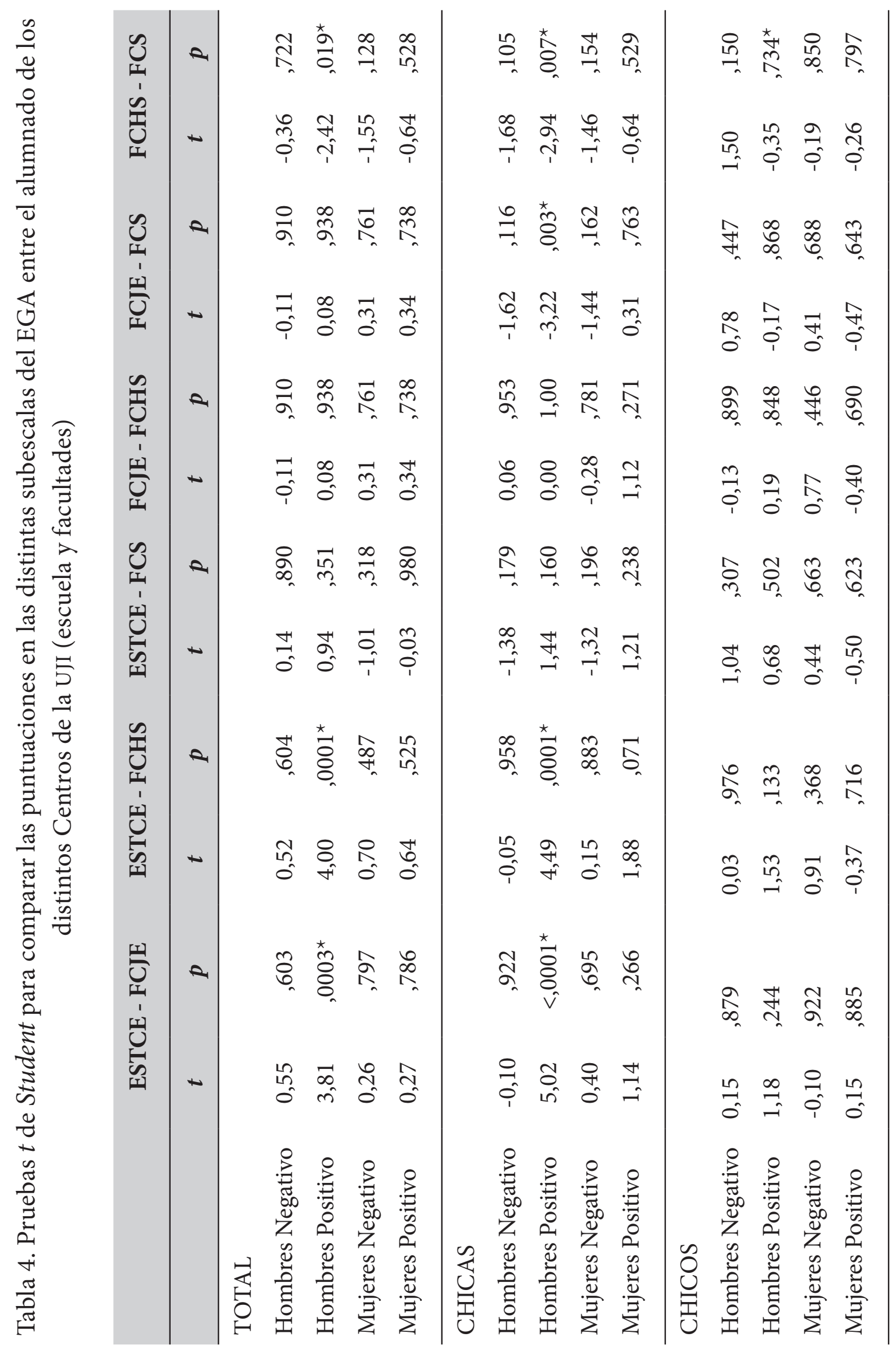




\section{Conclusiones}

La presente investigación pretendía explorar los estereotipos de género en una muestra representativa de deportistas universitarias/os de la Universitat Jaume I. Más concretamente, el objetivo de este trabajo era investigar si esta autopercepción de los estereotipos de género en personas que realizan práctica deportiva competitiva también influía en la elección de la carrera universitaria.

A priori, podemos observar que realizar práctica deportiva competitiva no influye en la elección de la carrera universitaria, ya que los resultados de las/ os deportistas muestran el mismo patrón de elección de carrera agrupados por centros comparado con los datos que recoge la UJI en su documento «La igualtat en xifres a l'UJI» (2018). Nuestros datos muestran una distribución muy similar: en la FCJE las mujeres representan el 55,6 \% y los hombres el 44,4 \%; en la ECTS las mujeres suponen el 31,9 \% y los hombres el 68,1\%; y en la FCS las mujeres representan el 71,4 \% y los hombres el 28,6\%. La única facultad que no perpetúa el patrón del informe de la UJI es la FCHS, ya que en nuestra muestra las mujeres suponen el 53 \% y los hombres el 46,7 \%. Por tanto, no replicaría el patrón de facultad más feminizada como indicaba el informe de la UjI. Asimismo, si observamos los grados que cursan los hombres deportistas de nuestra muestra observamos que corresponden a los grados menos feminizados dentro de dicha facultad (i.e., Magisterio de Primaria, Comunicación Audiovisual y Historia y Patrimonio). Por tanto, no se cumpliría nuestra primera hipótesis, según la cual esperábamos que la elección de carrera del estudiantado que practica deporte competitivo estuviera sesgada hacia carreras consideradas más masculinas, dado que se considera de dominio masculino.

Nuestra segunda hipótesis planteaba que hombres y mujeres puntuarían alto en los estereotipos pertenecientes a su rol de género. Si observamos nuestros datos comparando mujeres y hombres deportistas, sólo encontramos diferencias significativas en la subescala de Estereotipo de Hombre Negativo, con mayores puntuaciones para el grupo de hombres, un resultado congruente con lo esperado. Además, al comparar hombres y mujeres agrupados por facultades, se observó que en la FCHS los estereotipos de género repercuten negativamente en los hombres, ya que en una facultad con una mayoría de mujeres se distinguen de estas por la mayor puntuación significativa en los Estereotipos de Hombre Negativo. Por otra parte, en la ESTCE los estereotipos de género repercuten positivamente en las mujeres ya que en una facultad masculinizada se diferencian 
de los hombres por la puntuación mayor significativa en el Estereotipo de Mujer Positivo. Esto apuntaría que los y las universitarias que realizan práctica deportiva competitiva no poseen una interiorización muy arraigada de los estereotipos que condicionan sus roles de género.

Se esperaba que aquellos centros más masculinizados mostrasen valores más altos en estereotipos de género masculinos, mientras que los consideradas tradicionalmente como más femeninos diesen valores superiores en estereotipos de género femeninos. Esta hipótesis se cumple parcialmente ya que al comparar al estudiantado de la ESTCE frenta a la FCJE observamos diferencias significativas en la puntuación de Estereotipos de Hombre Positivo, mayor en la ESTCE. Observamos diferencias significativas acorde con la hipótesis, entre la ESTCE y la FCHS, con una mayor puntuación de la Escuela para el Estereotipo de Hombre Positivo, la FCS muestra valores significativos y mayores. También observamos diferencias significativas que no van en la línea de nuestra hipótesis ya que, cuando comparamos los valores obtenidos en la FCS con los de la FCJE y la FCHS, observamos que. en la subescala de Estereotipos de Hombre Positivo, la FCS muestra valores significatibvos y mayores. Para tratar de dar respuesta a nuestros resultados, realizamos una investigación más profunda comparando las diferentes facultades por género. Analizando las diferentes facultades por género observamos que no hay diferencias significativas para los hombres entre facultades, las diferencias significativas encontradas anteriormente pertenecen al grupo de las mujeres. La puntuación obtenida para el grupo de mujeres de la ESTCE para el Estereotipo de Hombre Positivo es mayor respecto al resto de facultades, acorde con lo esperado, ya que la ESTCE es una facultad masculinizada; por tanto, el grupo de mujeres puntuará alto en el estereotipo de hombre respecto el resto de facultades. En cambio, contrariamente a lo previsto en nuestra hipóstesis inicial el grupo de mujeres de la FCS obtuvo mayores puntuaciones respecto el grupo de mujeres de la FCJE y el grupo de mujeres de la FCHS, pese a que la FCS se considera más feminizada, es una facultad donde se premia la excelencia, que generalmente está asociada al rol masculino, esto podría explicar las elevadas puntuaciones para el estereotipo de Hombre Positivo del grupo de mujeres de la FCS.

Por tanto, nuestros datos, no parecen concluir que nuestras/os deportistas poseen una fuerte percepción de estereotipos acorde con su identidad de género. Sin embargo, la elección de carrera sí que está relacionada con la percepción del rol que ellos y ellas poseen. Esto podría explicarse en parte por la influencia de la familia y la sociedad en la elección de carrera, ya que su elección corresponde 
a los roles de género que perpetúa la sociedad. Además, el hecho de practicar deporte competitivo no influiría directamente en la elección de carrera, pero quizá sí que influye en la percepción de los estereotipos, ya que observamos que nuestros/as universitarios/as deportistas no siguen los patrones esperados.

Debemos señalar algunas limitaciones observadas en nuestra investigación. En primer lugar, cabe destacar que no existe un grupo control, por lo que sería interesante incluir un grupo equiparado en las características sociodemográficas pero que nunca haya practicado deporte de forma competitiva, lo que nos permitiría explorar con mayor fiabilidad qué cambios podrían atribuirse específicamente a la competición deportiva. Asimismo, como futuras líneas de investigación que den continuidad a este trabajo, en caso de diseñar nuevos estudios, se podrían incluir medidas que indiquen la influencia del contexto en los estereotipos, como pueden ser la familia y el grupo de amigos y/o amigas. Igualmente, podrían llevarse a cabo nuevos trabajos con otras poblaciones de estudio, como adolescentes o adultos de alto rendimiento, incluyendo personas que no tienen un perfil universitario pero realizan práctica deportiva competitiva, deportistas que han realizado práctica deportiva competitiva durante un período prolongado pero en el momento del estudio ya no lo practican, etc. Para finalizar, sería interesante desarrollar protocolos de intervención -probados en muestras de personas deportistas- con el fin de utilizar el deporte como herramienta para trabajar y combatir los sesgos negativos de los estereotipos y roles de género.

\section{Bibliografía}

Castillo-Mayén, Rosario, y Beatriz Montes-Berges. 2014. «Analysis of current gender stereotypes». Anales de Psicología 30: 1044-1060.

Clément-Guillotin, Corentin, Laurent Cambon, Aïna Chalabaev, Rémi Radel, Sophie Michel, y Paul Fontayne. 2013. «Social value and asymmetry of gender and sex categories in physical education». Revue Européenne de Psychologie Appliquée/European Review of Applied Psychology 63 (2): 75-85. doi: https:// doi.org/10.1016/j.erap.2012.12.004.

Chalabaeva, Aïna, Philippe Sarrazinb, Paul Fontaynea, Julie Boichéc, y Corentin Clément-Guillotin. 2013. "The influence of sex stereotypes and gender roles on participation and performance in sport and exercise: Review and future directions». Psychology of Sport and Exercise 14 (2): 136-144. 
González Gavaldón, Blanca. 1999. «Los estereotipos como factor de socialización en el género». Comunicar 12: 79-88. https://www.redalyc.org/pdf/158/15801212. pdf

Hardin, Marie, y Jennifer D. Greer. 2009. «The influence of gender-role socialization, media use and sports participation on perceptions of gender-appropriate sports». Journal of Sport Behavior 32: 207-226.

Mackie, Marlene. 1973. "Arriving at "truth" by definition: The case of stereotype inaccuracy». Social Problems 20 (4): 431-447.

Riemer, Brenda A., y Michelle E. Visio. 2003. «Gender typing of sports: an investigation of Metheny's classification». Research Quarterly Exercise and Sport 74 (2): 193-204. doi: https://doi.org/10.1080/02701367.2003.10609081.

Unitat d'Igualtat Universitat Jaume I. 2018. «La igualtat en xifres a l'UJI». Castelló de la Plana: Universitat Jaume I. https://documents.uji.es/alfresco/d/d/workspace/SpacesStore/2cbe3dc0-6e78-4095-b710-f56ed615d335/Z.+Igualtat+en+ xifres.+ALUMNAT+2017.2018.+08.06.2018.pdf?guest=true 
ÍNDEX 


\title{
Un programa de intervención en psicología positiva para aumentar el bienestar de los docentes
}

\author{
Marisa García Baldán
}

baldan@uji.es

La presente investigación tiene como finalidad analizar la relación entre los niveles de bienestar de un grupo de docentes no universitarios y la formación en psicología positiva aplicada a la educación, a través de un curso de formación del profesorado de treinta horas de duración, ofertado por el Cefire de Castellón. La hipótesis de la cual partimos es que, después de la realización del curso PositivitiEs, se produce un aumento en los niveles de bienestar de los docentes participantes. La muestra objeto del presente estudio está compuesta por un total de treinta docentes de diferentes centros educativos públicos de educación infantil y primaria de la provincia de Castellón. Se trata de un estudio experimental con dos momentos de medida. Durante esta investigación, el grupo de maestros y maestras participó en un curso de formación en psicología positiva aplicada a la educación. Un total de diez sesiones de tres horas cada una, durante un periodo de cinco semanas. Se midieron sus niveles de bienestar antes y después de la realización del curso PositivitiEs. Los datos se obtuvieron mediante el cuestionario PHI y el análisis de datos a través del paquete estadístico SPSS, uno de los programas informáticos más extendidos para el tratamiento estadístico de los datos en Ciencias Sociales y de la Salud. Los resultados ponen de manifiesto las diferencias entre los niveles del grupo pre-intervención y post-intervención. Es decir, observamos diferencias significativas entre los niveles de bienestar de los participantes antes y después de la realización del curso. 
Palabras clave: psicología positiva, docente, formación, educación, bienestar

\section{Introducción}

La Ley Orgánica 8/2013, de 9 de diciembre, para la mejora de la calidad educativa (LOMCE) establece, en su artículo 102, que «la formación permanente constituye un derecho y una obligación de todo el profesorado y una responsabilidad de las Administraciones educativas y de los propios centros». El curso de formación PositivitiEs: Psicología Positiva aplicada a la Educación se enmarca dentro de la oferta formativa de formación permanente al profesorado de la Conselleria d'Educació, Investigació, Cultura i Esport, de la Generalitat Valenciana y está dirigido a un grupo de treinta maestros y maestras de colegios educativos públicos de educación infantil y primaria de la provincia de Castellón. El objetivo de esta investigación es conocer su impacto en el bienestar de un grupo de docentes. El curso consta de diez sesiones de tres horas cada una, que se extienden a lo largo de un periodo de cinco semanas. Los niveles de bienestar se midieron antes y después de la realización del curso. Nuestra hipótesis es que, después de la realización del curso PositivitiEs, se produce un aumento en los niveles de bienestar de los docentes participantes.

En este capítulo, se presenta el marco teórico para contextualizar la psicología positiva aplicada a la educación y se enmarcan algunos de los contenidos relevantes de la psicología positiva presentes en los contenidos de PositivitiEs. Seguidamente, se detalla el objetivo de esta investigación y los objetivos específicos del curso. A continuación, se realiza una descripción de la metodología. Por último, se presentan los resultados de la investigación, para finalizar con unas conclusiones a modo de reflexión.

\section{Marco teórico}

La psicología positiva es el estudio científico del funcionamiento óptimo de las personas y las organizaciones. Su principal objetivo es comprender y facilitar 
la felicidad ${ }^{1}$ y el bienestar psicológico (Seligman 1998). El inicio formal de la psicología positiva se sitúa a finales del siglo pasado, concretamente, en 1998, de la mano de Martin Seligman (Cano Vindel 2014). La psicología positiva es una rama de la psicología que tiene como objetivo el estudio de los aspectos psicológicos positivos del ser humano. De este modo, la psicología positiva propone dar un giro en la investigación psicológica hacia los aspectos más saludables del ser humano (Hervás 2009).

El interés por el análisis del bienestar crece a mediados del siglo pasado a partir de las aportaciones de Martin Seligman. El estudio de lo positivo de la experiencia humana se puede entender desde tres ángulos diferentes: el análisis de las experiencias positivas, estados de bienestar psicológico, felicidad, estados de flujo o de experiencias óptimas, satisfacción con la vida, etc.; la aproximación al estudio de las fortalezas psicológicas; y el análisis de las características que configuran y determinan organizaciones positivas, como familia, escuela, empresa, barrio, etc. (Vázquez 2009).

El bienestar es el antídoto positivo contra la incidencia disparada de la depresión, permite aumentar la satisfacción con la vida y mejorar el aprendizaje (Seligman et al. 2009). Así pues, enseñar bienestar en la escuela, de manera sistemática y globalizada, en todos los momentos y espacios de aprendizaje, presenta ventajas multifacéticas. No obstante, para poder enseñar a los demás, el aprendizaje debe experimentarse personalmente. Cuando los propios alumnos y alumnas son protagonistas de su aprendizaje a partir del ejemplo directo y activo del docente, este último se convierte en modelo referente de aquello que deben aprender sus estudiantes.

A continuación, describiremos algunos de los constructos más significativos relacionados con la psicología positiva presentes en el curso PositivitiEs. En primer lugar, la atención plena o mindfulness, que puede definirse como «darse cuenta de la experiencia presente con curiosidad, apertura a la experiencia y aceptación» (Bishop et al. 2004). Así, la atención plena se concibe como una actitud permanente de consciencia y calma que nos permite vivir íntegramente en el momento presente.

1. A pesar de su popularidad, o quizá por eso mismo, el concepto felicidad es tan abierto y polisémico que no resulta muy apropiado como objeto de una indagación científica. Por eso, en el estudio haremos uso de un equivalente más preciso y menos controvertido: el término bienestar. 
Los estudios relativos a la práctica del mindfulness en educación ponen de manifiesto los beneficios tanto en los alumnos como en el profesorado (Meiklejohn et al. 2012). Por un lado, los beneficios de su práctica en los alumnos son:

- Mejora en la capacidad atencional y ejecutiva (memoria, planificación, organización y control de impulsos);

- Incremento en autorregulación emocional, habilidades sociales y preocupación por otros;

- Disminución de la ansiedad, la depresión y las emociones negativas;

- Sensación de calma, relajación y autoaceptación;

- Mejora de la autoestima y la calidad del sueño;

- Reducción de problemas conductuales de ira, impulsividad e hiperactividad.

Por otro lado, los beneficios de su práctica en los profesores son:

- Reducción de estrés y prevención del burnout;

- Mejora de la percepción de bienestar y la autoeficacia;

- Mejora de la salud cardiovascular;

- Disminución de errores a nivel cognitivo y del desgaste emocional;

- Mejora de la capacidad de respuesta a las necesidades de los alumnos y al clima en el aula.

Otro de los constructos importantes en psicología positiva es la educación emocional. El objetivo de la educación emocional es el desarrollo de competencias emocionales: conciencia emocional, regulación emocional, autogestión, inteligencia interpersonal, habilidades de vida y bienestar, etc. La práctica de la educación emocional en el aula implica diseñar programas fundamentados en su marco conceptual. Para llevarlos a la práctica hay que contar con profesorado debidamente preparado; para apoyar la labor del profesorado se necesitan materiales curriculares; para evaluar los programas se necesitan instrumentos de recogida de datos, etc. (Bisquerra 2003).

Dentro del marco conceptual en el que se ubica la educación emocional, destaca la noción de inteligencia emocional (IE). Salovey y Mayer introdujeron el término en 1990, definiéndola como la habilidad para percibir, comprender, asimilar y regular las emociones propias y las de los demás. De este modo, la IE 
ayuda a las personas a guiar sus pensamientos y reflexionar sobre sus emociones, contribuyendo a mejorar sus niveles de bienestar. Por ello, la inteligencia emocional está relacionada directamente con las emociones positivas y el bienestar psicológico (Fernández y Extremera 2009). Cabe señalar que el modelo teórico de Mayer y Salovey (1997) es el que recibe mayor acogida entre la comunidad científica. Los autores conciben la IE como una inteligencia genuina basada en el uso adaptativo de las emociones y su aplicación a nuestro pensamiento. De este modo, las emociones ayudan a resolver problemas y facilitar la adaptación al medio (Fernández y Extremera 2009).

Diferentes estudios ponen de manifiesto que los profesores con más inteligencia emocional experimentan menos desgaste personal (Extremera y Fernández-Berrocal 2004): menos cansancio emocional, menor grado de despersonalización y más realización personal. Además, para que el alumno aprenda y desarrolle las habilidades emocionales y afectos relacionados con el uso inteligente de sus emociones, necesita de "profesores emocionales». El profesor, sobre todo en las primeras etapas, se convierte en su referente más importante en cuanto a actitudes, comportamientos, emociones y sentimientos. Cabe puntualizar que tampoco debemos dejar toda la responsabilidad del desarrollo social del alumnado en manos de los docentes: la familia es el modelo emocional básico y conforma el primer espacio de socialización y educación emocional del niño. Por esta razón, padres y docentes deben complementarse en esta tarea y, de forma conjunta, proporcionar oportunidades para mejorar el perfil emocional del alumno (Extremera y Fernández-Berrocal 2004).

A continuación, haremos referencia a un novedoso constructo para definir el funcionamiento óptimo de las organizaciones y los trabajadores. Se trata del engagement, un estado psicológico positivo del empleado caracterizado por altos niveles de energía y vigor, dedicación y entusiasmo por el trabajo, así como total absorción y concentración en la actividad laboral (Salanova y Schaufeli 2004).

Al explorar las múltiples definiciones académicas que existen del término, es posible identificar dos aproximaciones generales. La primera lo asume como un rol laboral y sostiene que los trabajadores comprometidos se identifican con el trabajo y, por ello, se esfuerzan más. Según esta visión, el engagement corresponde a un tipo especial de conducta, en la que el trabajador está completamente inmerso en sus tareas (Salanova y Schaufeli 2004). De acuerdo con la segunda perspectiva, el engagement corresponde a un estado de realización del trabajador en la medida en que los trabajadores comprometidos sienten una conexión con su trabajo, porque lo perciben como un reto directivo y no como una carga 
(Salanova y Schaufeli 2004). Desde este enfoque, el engagement ha sido definido como un estado mental positivo, de realización, relacionado con el trabajo, que se caracteriza por el vigor, la dedicación y la absorción. El engagement de un trabajador depende en gran parte de sus conductas, sus creencias y sus motivaciones. En ese sentido, las organizaciones saludables se definen como aquellas que implementan el tipo de estrategias aquí descritas, para facilitar y promover el engagement de todos sus trabajadores (Salanova y Schaufeli 2004).

Siguiendo las aportaciones de Salonova y Schaufeli (2004), una de las dimensiones fundamentales del engagement es la absorción, relacionada con experiencias de flow; es decir, experiencias óptimas extremadamente disfrutadas en las que se da una atención focalizada, total concentración y disfrute, con un alto interés por la actividad en sí misma. Usualmente, estas experiencias implican la utilización de fortalezas personales y habilidades altamente desarrolladas y, a su vez, están vinculadas a altos niveles de felicidad, bienestar y desempeño.

Por otra parte, la coaching psychology es una rama de la psicología que se ocupa de la aplicación sistemática de la ciencia comportamental para la mejora de la experiencia vital, el rendimiento laboral y el bienestar de individuos, grupos y organizaciones (Grant 2007). Se centra en facilitar el logro de objetivos, mejorar el crecimiento y desarrollo personal y profesional de los clientes en su vida personal y en el ámbito de trabajo.

Siguiendo las aportaciones de Salanova, el PsiPos Coaching es aquel que parte de la investigación científica en psicología positiva y la traslada a instrumentos efectivos con el objetivo de ayudar a los clientes a aumentar su felicidad, bienestar y éxito (Salanova 2015). Los PsiPos Coaches utilizan los principios de la psicología positiva sobre el uso potencial de las fortalezas de los clientes (optimismo, gratitud, creatividad, etc.) para mejorar día a día su felicidad, que crece cuando experimentan emociones positivas, engagement, relaciones positivas, significado y consecución de objetivos.

Finalmente, mencionamos la educación positiva, que se define como la aplicación de la psicología positiva en el ámbito educativo. La educación positiva se dedica a investigar, proponer, implantar y divulgar las teorías y las técnicas científicamente validadas, aplicables al campo de la educación, siempre en un sentido amplio, abarcando instituciones educativas, educadores, alumnos y familias. Por este motivo, la educación positiva comprende cualquier concepto que mejore nuestra educación, nuestro funcionamiento óptimo, así como nuestra felicidad. Algunas áreas que abarca son la educación emocional, el optimismo, la creatividad, el sentido del humor, la motivación, el mindfulness, las fortalezas, 
la resiliencia, la gratitud, el entusiasmo, la autoestima, la asertividad o la compasión, entre otras (Taibo 2017).

Seligman señalaba, ya en 2009, tres razones por las que era necesario incluir la felicidad en la educación: un aumento del número de trastornos depresivos y una reducción de su edad de aparición; escaso incremento del nivel de felicidad de la población mundial; y la mejora del aprendizaje a través del bienestar. Otros científicos apuntaban también que las personas con mayor satisfacción de vida gozan de mejor salud física, mayor logro profesional, mejores relaciones sociales y mayores contribuciones económicas para su sociedad. En definitiva, la Educación Positiva logra mejorar la calidad de la educación y la prevención y solución de problemas relacionados con la salud mental (Taibo 2017).

\section{Objetivos}

El objetivo del presente estudio es averiguar si los docentes participantes en el grupo de formación han variado sus niveles de bienestar después de la realización de la misma. Para ello, la formación desarrolla competencias alineadas con el marco teórico expuesto y que se articulan en torno a los siguientes objetivos específicos del curso PositivitiEs:

- Formar al profesorado en psicología positiva aplicada a la educación.

- Formar al profesorado para que experimente algunas técnicas de psicología positiva (véase Arguís et al 2011).

- Atención plena

- Test de fortalezas

- Técnica de las 3 bendiciones

- La rueda de la vida

- Promover la importancia y la necesidad de la innovación en la práctica educativa a partir de psicología positiva aplicada en la educación.

- Dar a conocer los fundamentos científicos básicos de la psicología positiva. 
- Generar un cambio metodológico para mejorar el proceso de enseñanzaaprendizaje a partir de los fundamentos de la psicología positiva.

- Dar a conocer recursos didácticos, pedagógicos, estrategias y soporte especializado para desarrollar y aplicar un proyecto de psicología positiva aplicada a la educación en el centro educativo.

- Establecer una red de trabajo y colaboración abierta con otros centros educativos, instituciones y profesionales de psicología positiva en el ámbito educativo.

\section{Material y método}

En el primer trimestre escolar, se llevó a cabo un curso de formación en psicología positiva aplicada a la educación con un grupo de docentes de diferentes escuelas públicas de la provincia de Castellón. La muestra de la investigación se realizó, concretamente, a treinta docentes de educación infantil y primaria.

El curso PositivitiEs: psicología positiva aplicada a la educación formaba parte de la oferta formativa al profesorado del Cefire de Castellón. Los Centros de formación, innovación y recursos educativos (Cefire) de la Comunidad Valenciana dependen de la Conselleria d'Educació, Investigació Cultural i Esports de la Generalitat Valenciana y su principal función es la formación permanente y gratuita del profesorado de enseñanza no universitaria. De este modo, los Cefire tienen como finalidad favorecer la formación del docente como profesional, exigida por el actual sistema educativo (véase Pla Ferrer 2019). Así queda reflejado en el artículo 102 de la LOMCE, referente a la formación permanente del profesorado en centros públicos, en el cual se establece que las administraciones educativas planificarán las actividades de formación del profesorado, garantizarán una oferta diversificada y gratuita de estas actividades y establecerán las medidas oportunas para favorecer la participación del profesorado en ellas.

Cabe señalar que la realización del curso se llevó a cabo durante un primer trimestre escolar en un total de diez sesiones de tres horas cada una, durante cinco semanas. Los contenidos del curso fueron los siguientes:

- Introducción a la psicología positiva

- Mindfulness: atención y consciencia plena 
- Educación emocional: regulación emocional y emociones positivas

- Motivación

- Engagement y sentido

- Flow, experiencias óptimas

- Coaching y educación

- Hacia una educación positiva

Los datos de la muestra se obtuvieron mediante el cuestionario Pemberton Happiness Index (PHI) elaborado por los investigadores Carmelo Vázquez y Gonzalo Hervás. Se suministró a los participantes el cuestionario antes y después de la intervención, para conocer los niveles de bienestar en ambos momentos. El Índice Pemberton de Felicidad (PHI; Hervás y Vázquez 2013) ofrece una medida individual de felicidad experimentada y, también, recordada, lo cual lo diferencia de anteriores propuestas. Además, tiene en cuenta tanto componentes eudaimónicos como hedónicos en el nivel de felicidad, por lo que supone una medida muy completa.

El bienestar psicológico resulta una construcción compleja, que incluye diferentes perspectivas. Este índice recoge los avances más importantes y genera una medida equilibrada que refleja el constructo latente de bienestar. Para ello, emplea diferentes indicadores para medir el bienestar recordado y el bienestar experimentado. Todo ello, de forma sintética, en once ítems.

\section{Resultados y análisis}

Los resultados se obtuvieron a partir de los datos cuantitativos obtenidos de la muestra: treinta maestros y maestras. Estos datos se midieron mediante el cuestionario PHI y el análisis de datos a través del paquete estadístico SPSS, uno de los programas informáticos más extendidos para el tratamiento estadístico de los datos en Ciencias Sociales y de la Salud.

Los datos obtenidos muestran que los niveles de bienestar de los sujetos después de la intervención, son significativamente mayores que los niveles iniciales $\mathrm{F}(1,12)=55,08, p<, 01$, (media después de la intervención $=8,12$, media inicial $=7,26)$. 
Los resultados ponen de manifiesto las diferencias entre los niveles de bienestar del grupo pre-intervención y el grupo post-intervención. Así pues, observamos divergencias significativas entre los niveles de bienestar de los participantes antes y después de la realización del curso PositivitiEs: psicología positiva aplicada a la educación.

Cabe señalar que, aunque los resultados ponen de manifiesto la variación entre el primer y el segundo momento de medición, los docentes participantes en la medición pre-intervención muestran ya niveles notables de bienestar.

\section{Conclusiones}

El objetivo de la investigación aquí descrita era conocer el impacto en los niveles de bienestar de un grupo de maestros y maestras después de recibir un curso de formación en psicología positiva aplicada a la educación. El curso se extendió a lo largo de un total de diez sesiones de tres horas cada una, durante un periodo de cinco semanas. Se midieron los niveles de bienestar antes y después de la realización del curso y los datos obtenidos a partir del análisis confirman la hipótesis: los niveles de bienestar aumentan después de la realización del curso PositivitiEs: psicología positiva aplicada a la educación.

La línea de investigación próxima es diseñar y aplicar un programa de intervención en psicología positiva para aumentar el bienestar de los estudiantes del grado de maestros y maestras de educación primaria de la Universitat Jaume I. El propósito es contribuir a construir escuelas positivas, aportando un poco más de bienestar a las generaciones futuras y al conjunto de nuestra sociedad.

\section{Bibliografía}

Arguís, Ricardo, Ana Pilar Bolsas, Silvia Hernández y María del Mar Salvado. 2011. «Programa aulas felices. La psicología positiva en las aulas.» Revista del Museo Pedagógico de Aragón 3: 52-57. http://cepgranada.org/ inicio/archivos/ ar3061_programa \%20aulas \%20felices.pdf

Bishop, Scott R. et al. 2006. «Mindfulness: A proposed operational definition». Clinical Psychology Science and Practice 11 (3): 230-241. 
Bisquerra, Rafael. 2003. «Educación emocional y competencias básicas para la vida». Revista de Investigación Educativa 21: 7-43.

Cano Vindel, Antonio. 2014. «¿Podemos medir la felicidad?» Boletín de la SEAS 40. http://www.ansiedadyestres.org/content/entrevista-b-40

Extremera, Natalio y Pablo Fernández-Berrocal. 2004. «La importancia de desarrollar la inteligencia emocional en el profesorado». Revista Iberoamericana de Educación 33: 1-9.

Fernández-Berrocal, Pablo y Natalio Extremera. 2009. «La inteligencia emocional y el estudio de la felicidad». Revista Interuniversitaria de Formación del Profesorado 23: 85-108.

Grant, Suzanne. 2007. «Learning through 'being' and 'doing'». Action Research 5 (3): 265-274.

Hervás, Gonzalo. 2009. «Psicología positiva: una introducción». Revista Interuniversitaria de Formación del Profesorado 23 (3): 23-41. http://m.aufop.com/ aufop/uploaded_files/articulos/1258587094.pdf

Ley Orgánica 8/2013, de 9 de diciembre, para la mejora de la calidad educativa (LOMCE).

Mayer, John D. y Peter Salovey. 1997. «What is emotional intelligence?» En Emotional Development and Emotional Intelligence: Implications for Educators, editado por Peter Salovey y David Sluyter, 3-31. Nueva York: Basic Books.

Meiklejohn, John, Catherine Philips, M. Lee Freedman, et al. 2012. Integrating Mindfulness Training into K-12 Education: Fostering the Resilience of Teachers and Students. https://greatergood.berkeley.edu/images/uploads/Integrating_ Mindfulness_Training_Into_K-12_Education.pdf

Pla Ferrer, Josep. 2019. «Norma inicial». Mestre a casa. http://mestreacasa.gva.es/ web/cefirevinaros/cefire

Salanova Soria, Marisa y Wilmar B. Schaufeli. 2004. «El engagement de los empleados: un reto emergente para la dirección de los recursos humanos». Estudios Financieros 261: 109-138.

Salanova, Marisa. 2015. «Psicología positiva y coaching: ¿Un maridaje saludable?». El Blog de Marisa Salanova (Smiling :). http://marisasalanova.blogspot. com/2015/02/psicologia-positiva-y-coaching-un.html.

Salovey, Peter y John D. Mayer. 1990. «Emotional intelligence». Imagination, Cognition, and Personality 9: 185-211.

Seligman, Martin Elias Pete. 1998. Learned Optimism. Nueva York: Pocket Books. 
Seligman, Martin Elias Pete, Randal M. Ernst, Jane Gillham, Karen Reivich, and Mark Linkins. 2009. «Positive education: Positive psychology and classroom interventions». Oxford Review of Education 35 (3): 293-311.

Taibo, Iago. 2017. «Educación positiva: La propuesta científica para una sociedad feliz». Educación 3.0. https://www.educaciontrespuntocero.com/opinion/ educacion-positiva-propuesta-cientifica-sociedad-feliz/52645.html

Vázquez, Carmelo. 2009. La ciencia del bienestar: Fundamentos de una psicología positiva. Madrid: Alianza Editorial. 


\section{Notes biogràfiques}

Marisol C. Bock és ciutadana alemanya i veneçolana amb residència a Espanya. Va cursar els seus estudis de grau en Art i Cultura a la Universitat de Maastricht (2008) i els seus estudis de màster a la Càtedra UNESCO de Filosofia de la Pau, Conflictes i Desenvolupament de la Universitat Jaume I (2015). Actualment realitza els seus estudis de doctorat en decreixement econòmic sostenible des d'una perspectiva ecofeminista. Entre els seus interessos de recerca s'inclouen els estudis de la pau i el conflicte, els estudis de gènere, els estudis queer, lecologia de la pau i els enfocaments transracionals de la pau. El 2015, va ingressar a la Global DEEP Network, una comunitat mundial de creació de canvi els membres de la qual estan compromesos en la construcció d’un món pacífic i ecològicament regeneratiu (globaldeepnetwork. org). En aquest context, ha facilitat projectes educatius sobre violència de gènere, estudis de la pau per a docents i encarnació de la pau i la música com a vehicle per a la pau. També forma part del projecte de música per a l'educació en la pau Rosalía Mowgli i La Escuela Encantada (rosaliamowgli.com).

Samantha N. Edwards és estudiant de doctorat a la Universitat de Cambridge, Regne Unit, on treballa en la tesi «News es Activism: Communicating Armed Resistance in Wartime» ('Les notícies com activisme: comunicar la resistència armada en temps de guerra'). Actualment és investigadora invitada a la Universitat Jaume I. Té un màster en Comunicació per la Universitat de Utah i un màster en Traducció Literària com a Pràctica Creativa per la Universitat d'Edimburg. En els seus estudis de grau, va obtenir una llicenciatura en Literatura Comparada i una llicenciatura en estudis Luso-Brasilers del Smith College. Té una àmplia experiència com a traductora i directora de projectes de traducció i com a professora d'idiomes, comunicació i cultura. Els seus interessos de recerca inclouen la traducció literària, els estudis de comunicació i els estudis de gènere. 
María Escrig Mateu és graduada en Psicologia per la Universitat Jaume I (2019). Va realitzar les pràctiques curriculars en el Centre d'Especialització de Malaltia Mental (CEEM) La Bartola. El seu Treball de Fi de Grau, «Benestar psicològic en esportistes universitaris/àries de la Universitat Jaume I», estava emmarcat en un projecte finançat per la Càtedra Endavant Villarreal CF de l'Esport.

Camilo Eduardo Espinosa Díaz (Cartagena, Colombia, 1989) és comunicador social. Va obtenir un màster en Govern i Polítiques Públiques de la Universitat EAFIT (Colòmbia) i un màster en Estudis Internacionals de Pau, Conflictes i Desenvolupament de la Universitat Jaume I. En l'actualitat, realitza estudis de doctorat en Estudis Internacionals de Pau, Conflictes i Desenvolupament a la Universitat Jaume I i de Ciències Socials, amb èmfasi en Estudis Llatinoamericans, a la Universitat de Salamanca. És membre del grup de recerca «Dones, pràctiques culturals i gènere» de la Universitat de Cartagena (Colòmbia). Ha participat en importants conferències i congressos de Ciència Política a Anglaterra, França, Espanya, Mèxic i Equador, entre d'altres.

Marisa García Baldán es va iniciar en la docència com a mestra d’educació infantil i primària en una escola privada a Barcelona, on va nàixer. Durant més de dotze anys, també ha exercit a la Comunitat Valenciana com a mestra de l'escola pública. Va cursar el màster en Comunicació Intercultural i Ensenyament de Llengües i el màster en Psicologia Positiva Aplicada a la Universitat Jaume I. Actualment, és professora associada al Departament de Pedagogia i Didàctica de les Ciències Socials, la Llengua i la Literatura de la mateixa Universitat Jaume I. Com a experta en psicologia positiva, la seua recerca versa sobre el lideratge i el benestar en l'àmbit educatiu. Ha dissenyat i està aplicant un programa d'intervenció en psicologia positiva per augmentar el benestar dels estudiants universitaris, concretament en els estudiants del grau de mestres d'educació primària, els futurs professionals de l'educació, principals arquitectes d'escoles positives.

Gaetano Giannotta (Palermo, 1991) és graduat en Patrimoni Cultural per la Università degli Studi di Palermo (2016). Va obtenir el màster en Història de l'Art per la mateixa universitat el 2018, amb la qualificació d'Excel-lent 
Cum Laude. Des d’octubre de 2018 és doctorand en Història de l'Art per la Universitat Jaume I de Castelló i la Universitat de València, on desenvolupa un projecte de tesi doctoral sobre la decoració en estuc a la València del segle XVIII i els seus vincles tècnics i estilístics amb Itàlia. Des de l'abril de 2019, és membre del projecte de restauració de l'església dels Sants Joans de València, finançat per la Fundació Hortensia Herrero i coordinat per la professora Pilar Roig Picazo. Les seues publicacions i participacions en congressos versen sobre la independència de la decoració, els seus vincles iconogràfics, conservació i valorització.

Carlos Hernando Domingo és diplomat universitari en Infermeria per la Universitat de València, llicenciat en Educació Física a l'INEF de Barcelona i doctor en Educació per la Universitat Jaume I. És professor titular d'Universitat en el Departament d'Educació i Didàctiques Específiques. Des del 1992 dirigeix el Servei d'Esports de la Universitat Jaume I, servei que el 2004 es va convertir en el primer servei d'esports espanyol universitari a obtenir una certificació ISO9001. Ha dirigit dues tesis doctorals, ha participat com a ponent en diferents reunions científiques i ha publicat diversos articles en l'àmbit del rendiment en proves de llarga durada, de l'esport universitari i de la didàctica de l'educació física. Ha participat com a investigador principal en diferents projectes (Penyagolosa Trail Saludable PTS, Corre Recupera Repite Siempre Saludable CRS, Costa Blanca Trail Saludable CBTS, entre d'altres).

Jahel Molina Ulldemolins és estudiant de doctorat del programa de Psicologia de l'Esport i la Salut de la Universitat Autònoma de Barcelona i graduada en Psicologia per la Universitat Jaume I (2017), on també va cursar estudis de màster en el programa d'Investigació Aplicada en Estudis Feministes, de Gènere i Ciutadania. Va obtenir el grau de Màster en Psicologia de l'Esport i l'Exercici (2018) pel Col-legi Oficial de Psicologia de la Comunitat Valenciana. Treballa com a tècnica i coordinadora de projectes a la Federació d'Associació d'Estudiants FADES i col-labora amb el grup de recerca MPAGER de la Universitat Jaume I.

Esther Monzó-Nebot és professora titular del Departament de Traducció i Comunicació de la Universitat Jaume I. Entre el 2013 i el 2015 es va dedicar a la formació en investigació al Departament d'Estudis de la Traducció 
i la Interpretació de la Universitat de Graz (Àustria), com a catedràtica de Sociologia de la Traducció i la Interpretació. La seua recerca se centra en com la traducció i la interpretació contribueixen a la gestió de la diversitat en les societats i organitzacions actuals i en com traductores i intèrprets influeixen o poden influir en els desequilibris de poder estructural i social. Amb enfocaments psicosocials, realitza estudis descriptius sobre com els habitus i l'agència de traductores i intèrprets construeixen les cultures de la traducció i la interpretació. En aquest context, s'interessa per com la competència intergrupal pot millorar el treball de traductores i intèrprets en la comunicació i les relacions entre cultures i grups socials. La doctora Monzó és membre de l'Institut Interuniversitari de Filologia Valenciana i directora del màster universitari en Investigació en Traducció i Interpretació.

Joan Alfred Noll Obiol s'ha procurat una educació multidisciplinària amb uns graus inicials en Interpretació Musical (clarinet), Enginyeria Tècnica de Telecomunicacions i Sonologia. Malgrat haver començat la seua carrera professional com a programador informàtic, es va enfocar més tard cap a l'educació i va obtenir una plaça com a funcionari a la Generalitat de Catalunya, amb la qual imparteix classes tant online com presencialment. Després d'adonar-se de la rellevància dels llenguatges i la traducció en qualsevol àmbit del coneixement humà, va obtenir un Màster en Escriptura Creativa (Universitat Pompeu Fabra) i un Master of Science en Traducció Literària (University of Edinburgh), amb una tesi centrada en la traducció d'un musical de Sondheim. Actualment treballa en la seua tesi doctoral sobre traducció enllaçada amb la música a la Universitat Jaume I, tasca que compagina amb la seua feina com a professor.

M. Carmen Pastor Verchili és doctora en psicologia per la Universitat Jaume I i professora titular de psicologia bàsica del Departament de Psicologia Bàsica, Clínica i Psicobiologia de la mateixa universitat. És coordinadora del grup de recerca MPAGER de la Universitat Jaume I des de desembre de 2014. Les seues principals línies de recerca bàsica són l'estudi dels sistemes cerebrals implicats en el processament d'estímuls emocionals, i en les reaccions psicofisiològiques i conductuals davant d’aquests estímuls en mostres normals, clíniques i subclíniques. Ha treballat en l'adaptació a la població espanyola d'instruments per a l'estudi de les emocions en el laboratori, així 
com instruments diagnòstics en l'àmbit de la psicopatia i la regulació emocional. Pertany a societats científiques en l'àmbit de la neurociència cognitiva i afectiva (SEPNECA, SPR, APS), és membre afiliat a Europa de l'NIMH-CSEA de la Universitat de Florida i de l'Institut Universitari d'Estudis Feministes i de Gènere «Purificación Escribano» (Universitat Jaume I).

Irina Charlotte Ranaivosoa va cursar una llicenciatura en Dret a la Universitat de Tolosa, França, i un Màster en Dret a la Universitat de Birmingham, Regne Unit. La seua tesi de màster es va centrar en l'aplicació del dret humà a la pau en el dret internacional. Actualment cursa estudis de pau i ciències socials en el Màster Internacional d'Estudis per la Pau, Conflictes i Desenvolupament de la Universitat Jaume I, Espanya. El seu principal interès d'investigació és l'estat i la implementació de la cultura de la pau a França, tema en el qual té previst centrar els seus estudis de doctorat. Ha treballat com a assistent de projectes per al Col-lectiu per a la Defensa de les Terres de Madagascar (Tany) i ha ocupat diversos càrrecs en mitjans socials, comunicació i desenvolupament de projectes comunitaris centrats en la salut i l'educació de la joventut al Regne Unit, Senegal i Vietnam.

Sonia Reverter Bañón és doctora en filosofia, professora titular de filosofia del Departament de Filosofia i Sociologia de la Universitat Jaume I i directora de l'Institut d'Investigació Feminista «Purificación Escribano» de la Universitat Jaume I. Ha estat professora convidada a les universitats de Berkeley (USA), European Peace University (Àustria), European Peace University (Ireland), Universidad Autónoma del Estado de México, Massachusetts Institute of Technology (USA), University of Limerick (Ireland), Peace and Conflict Research Institute (Dinamarca), International Folk School (Dinamarca), University of San Francisco (USA), Gender Institute de London School of Economics (Regne Unit), Centre for Civil Society de London School of Economics (Regne Unit), Universidad Pública de Pelotas (Brasil) i Universidad Nacional del Comahue (Argentina). Les seues línies d'investigació prioritàries són la teoria feminista, la crisi del pensament europeu contemporani, la societat civil, la teoria política feminista, el ciberfeminisme, ciutadania i identitat i el neurogènere. 
Ana Vázquez Fernández va estudiar Traducció i Interpretació a la Universitat de Salamanca, on es va especialitzar en interpretació. Té un Màster en Investigació en Traducció i Interpretació per la Universitat Jaume I i actualment està acabant el Màster en Llengües Estrangeres i Lingüística a la Universität Potsdam. La seua experiència en traducció s'ha desenvolupat a Espanya, Portugal i actualment a Alemanya. Els seus interessos de recerca en els estudis de traducció i interpretació inclouen la perspectiva de gènere, els enfocaments bibliomètrics, el postcolonialisme i la interseccionalitat. També estudia la comunicació de la ciència i les interseccions entre les ciències naturals i tecnològiques i les ciències socials i humanes. 


\section{Bionotes}

Marisol C. Bock is a German and Venezuelan citizen living in Spain. She completed her BA in Arts and Culture at Maastricht University in 2008 and obtained her MA in 2015 at the UNESCO Chair of Philosophy for Peace in Peace, Conflict and Development at Universitat Jaume I. She is currently conducting her $\mathrm{PhD}$ research on sustainable economic degrowth from an ecofeminist perspective. Her research interests include peace and conflict studies, gender studies, queer studies, peace ecology, and transrational approaches to peace. In 2015, Marisol joined the Global DEEP Network, a global community of change makers committed to building a peaceful and ecologically regenerative world (globaldeepnetwork.org). In this context, she has facilitated educational projects on gender-based violence, peace studies for teachers, peace embodiment, and music as a vehicle for peace. She also belongs to the music for peace education project Rosalía Mowgli and the Enchanted School (rosaliamowgli.com).

Samantha N. Edwards is a $\mathrm{PhD}$ student at the University of Cambridge, $\mathrm{UK}$, where she is working on her thesis «News as Activism: Communicating Armed Resistance in Wartime». She is currently a visiting researcher at Universitat Jaume I. She holds a Master's degree in Communication from the University of Utah and a Master's of Science in Literary Trnaslation as Creative Practice from the University of Edinburgh. In her undergraduate studies, she obtained a BA in Comparative Literature and a BA in PortugueseBrazilian Studies from Smith College. She has extensive experiences as a translator and translation manager and as a lecturer in languages, communication, and culture. Her research interests include literary translation, communication studies and gender studies.

María Escrig Mateu has a degree in Psychology from Universitat Jaume I (2019). She conducted a curricular internship at the Centro de 
Especialización de Enfermedad Mental (CEEM) La Bartola. Her Final Degree Essay, «Bienestar psicológico en deportistas universitarios/as de la Universitat Jaume I» (Psychological Well-being in University Sportsmen and Women at Universitat Jaume I), was part of a project funded by the Endavant Villarreal CF Chair of Sport.

Camilo Eduardo Espinosa Díaz (Cartagena, Colombia, 1989) is a social communicator. He obtained a Master's degree in Governance and Public Policy from EAFIT University (Colombia) and a Master's degree in International Peace, Conflict and Development Studies from Universitat Jaume I. He is currently conducting his doctorate studies in International Peace, Conflict and Development Studies at Universitat Jaume I and in Social Sciences, with an emphasis on Latin American Studies, at the University of Salamanca. $\mathrm{He}$ is a member of the research group Mujeres, prácticas culturales y género (Women, cultural practices and gender) at the University of Cartagena (Colombia). He has participated in important conferences and congresses in the area of Political Science in England, France, Spain, Mexico, and Ecuador, among others.

Marisa García Baldán began teaching as a kindergarten and primary school teacher in a private school in Barcelona, where she was born. For more than twelve years, she has also worked in the Valencian Community as a teacher in public schools. She completed her Master's Degree in Intercultural Communication and Language Teaching and a Master's Degree in Applied Positive Psychology at Universitat Jaume I. She is a part-time lecturer in the Department of Pedagogy and Didactics of Social Sciences, Language and Literature at the same University. As an expert in positive psychology, her research focuses on leadership and well-being in the educational field. She has designed and is implementing a program of intervention in positive psychology to increase the well-being of university students, specifically students in undergraduate programs in primary education, the future education professionals and principal architects of positive schools.

Gaetano Giannotta (Palermo, 1991) graduated in Cultural Heritage at the Università degli Studi di Palermo (Italy, 2016). He obtained a Master's degree in Art History from the same university in 2018, with the qualification of 
Cum Laude. From October 2018 he is a doctoral candidate in Art History at Universitat Jaume I and Universitat de València, where he is developing a doctoral project on stucco decoration in Valencia in the 18th century and its technical and stylistic links with Italy. Since April 2019 he has been a member of the restoration project of the Santos Juanes church in Valencia, funded by the Hortensia Herrero Foundation and coordinated by Professor Pilar Roig Picazo. His publications and conference participations deal with the independence of decoration, its iconographic links, its conservation and its valorization.

Carlos Hernando Domingo has a university diploma in Nursing from the University of Valencia, a degree in Physical Education from the INEF in Barcelona and a doctorate in Education from Universitat Jaume I. He is an Associate Professor at the University in the Department of Education and Specific Didactics. Since 1992 he has directed the Sports Service at Universitat Jaume I, a service that became the first Spanish university sports service to obtain ISO9001 certification in 2004. He has supervised two doctoral dissertations, has participated as a speaker in different scientific meetings and has published several articles in the field of performance in long-term tests, university sport and didactics of Physical Education. He has participated as principal investigator in different projects (Penyagolosa Trail Saludable PTS, Corre Recupera Repite Siempre Saludable CRS, Costa Blanca Trail Saludable CBTS, among others).

Jahel Molina Ulldemolins is a doctoral student in the Psychology of Sport and Health programme at the Autonomous University of Barcelona. She graduated in Psychology from Universitat Jaume I (2017), where she also studied a master's degree in Applied Research in Feminist, Gender and Citizenship Studies. She obtained a Master's degree in Sports and Exercise Psychology (2018) from the Colegio Oficial de Psicología de la Comunidad Valenciana (Chartered Institute of Psychology of the Valencian Community). She works as a technician and project coordinator for the Federation of Students' Association FADES and collaborates with the research group MPAGER of the University Jaume I. 
Esther Monzó-Nebot is an Associate Professor in the Department of Translation and Communication Studies at Universitat Jaume I. Between 2013 and 2015 she trained researchers in the field of sociology of translation and interpreting at the Department of Translation Studies of the University of Graz, Austria, as a full Professor. Her current research focuses on the ways in which translation and interpreting are contributing to the management of diversity in current societies and organizations and how translators and interpreters impact or may impact structural and social power imbalances. Her research uses psychosocial approaches to describe how translators' habitus and agency construe current translation cultures and how intergroup competence may enhance translators' work in intergroup communication and relations. Dr. Monzó is a member of the Institut Interuniversitari de Filologia Valenciana and director of the graduate program in Translation and Interpreting Research.

Joan Alfred Noll Obiol has pursued a multidisciplinary education with initial degrees in Musical Interpretation (clarinet), Technical Engineering in Telecommunications, and Sonology. Although he began his professional career as a computer programmer, he later focused on education and obtained a permanent position at the Generalitat de Catalunya, where he delivers both online and face-to-face instruction. After realizing the relevance of languages and translation in any field of human knowledge, he obtained a Master's in Creative Writing (Pompeu Fabra University) and a Master of Science in Literary Translation (Edinburgh University), with a thesis focused on the translation of a musical from Sondheim. He is currently working on his doctoral thesis on music-linked translation at Universitat Jaume I, a task that he combines with his work as a teacher.

M. Carmen Pastor Verchili holds a PhD in Psychology from Universitat Jaume I and is an Associate Professor in Basic Psychology in the Department of Basic Psychology, Clinical Psychology and Psychobiology at the same university. She has been the director of the MPAGER research group at Universitat Jaume I since December 2014. Her main lines of basic research are the study of the brain systems involved in the processing of emotional stimuli and in the psychophysiological and behavioural reactions to these stimuli in normal, clinical and subclinical samples. She has worked on 
the adaptation to the Spanish population of instruments for the study of emotions in the laboratory, as well as diagnostic instruments in the field of psychopathy and emotional regulation. She belongs to scientific societies in the field of cognitive and affective neuroscience (SEPNECA, SPR, APS), is an affiliated member in Europe of the NIMH-CSEA of the University of Florida, and of the University Institute of Feminist and Gender Studies «Purificación Escribano» (Universitat Jaume I).

Irina Charlotte Ranaivosoa completed a Licence de Droit (Bachelor of Law) at the University of Toulouse, France, and a LLM (Master of Laws) at the University of Birmingham, UK. Her master thesis focused on the implementation of the human right to peace in international law. She is currently pursuing peace and social sciences training in the International Master's in Peace, Conflict and Development Studies at the Universitat Jaume I, Spain. Her main research interest is the state and implementation of the culture of peace in France, the topic she is planning on focusing her doctorate studies. She has worked as a project assistant for the Collective for the Defense of Malagasy Lands - TANY and held various positions in social media, communication and project development officer in community-based projects focusing on health and youth education in the UK, Senegal, and Vietnam.

Sonia Reverter Bañón holds a PhD in Philosophy, is an Associate Professor in Philosophy in the Department of Philosophy and Sociology at Universitat Jaume I and is the Director of the Feminist Research Institute (IF) at Universitat Jaume I. She has been a guest lecturer at the universities of Berkeley (USA), European Peace University (Austria), European Peace University (Ireland), Universidad Autónoma del Estado de México, Massachusets Institute of Technology (USA), University of Limerick (Ireland), Peace and Conflict Research Institute (Denmark), International Folk School (Denmark), University of San Francisco (USA), Gender Institute of London School of Economics (United Kingdom), Centre for Civil Society of London School of Economics (United Kingdom), Universidad Pública de Pelotas (Brazil), and Universidad Nacional del Comahue (Argentina). Her main areas of research are feminist theory, the crisis of contemporary European thought, civil society, feminist political theory, cyber feminism, citizenship and identity, and neurogender. 
Ana Vázquez Fernández studied Translation and Interpreting at the University of Salamanca, where she specialised in interpreting. She holds a Master's in Research in Translation and Interpreting from the University Jaume I and is currently completing the Master's in Foreign Languages and Linguistics from Universität Potsdam. Her experience in translation has been developed in Spain, Portugal, and currently Germany. Her research interests within Translation and Interpreting Studies include the gender perspective, bibliometric approaches, post-colonialism, and intersectionality. She is also interested in the communication of science and the intersections between natural and technological sciences and social sciences and humanities. 


\section{Notas biográficas}

Marisol C. Bock es ciudadana alemana y venezolana con residencia en España. Cursó sus estudios de grado en Arte y Cultura en la Universidad de Maastricht (2008) y sus estudios de máster en la Cátedra UNESCO de Filosofía de la Paz, Conflictos y Desarrollo en la Universitat Jaume I (2015). Actualmente está realizando sus estudios de doctorado en decrecimiento económico sostenible desde una perspectiva ecofeminista. Entre sus intereses de investigación, se incluyen los estudios de la paz y el conflicto, los estudios de género, los estudios queer, la ecología de la paz y los enfoques transracionales de la paz. En 2015, ingresó en la Global DEEP Network, una comunidad global de creadores de cambio comprometidos con la construcción de un mundo pacífico y ecológicamente regenerativo (globaldeepnetwork.org). En ese contexto, ha facilitado proyectos educativos sobre violencia de género, estudios de paz para profesores, encarnación de la paz y la música como vehículo para la paz. También forma parte del proyecto de música para la educación en la paz Rosalía Mowgli y La Escuela Encantada (rosaliamowgli.com).

Samantha N. Edwards es estudiante de doctorado en la Universidad de Cambridge, Reino Unido, donde está trabajando en su tesis «News as Activism: Communicating Armed Resistance in Wartime» ('Las noticias como activismo: comunicar la resistencia armada en tiempo de guerra’). Actualmente es investigadora visitante en la Universitat Jaume I. Tiene un máster en Comunicación por la Universidad de Utah y un máster en en Traducción Literaria como Práctica Creativa por la Universidad de Edimburgo. En sus estudios de grado, obtuvo una licenciatura en Literatura Comparada y una licenciatura en Estudios Luso-Brasileños del Smith College. Tiene una amplia experiencia como traductora y directora de proyectos de traducción y como profesora de idiomas, comunicación y cultura. Sus intereses de investigación incluyen la traducción literaria, los estudios de comunicación y los estudios de género. 
María Escrig Mateu es graduada en Psicología por la Universitat Jaume I (2019). Realizó las prácticas curriculares en el Centro de Especialización de Enfermedad Mental (CEEM) La Bartola. Su Trabajo de Fin de Grado, «Bienestar psicológico en deportistas universitarios/as de la Universitat Jaume I», estaba enmarcado en un proyecto financiado por la Cátedra Endavant Villarreal CF del Deporte.

Camilo Eduardo Espinosa Díaz (Cartagena, Colombia, 1989) es comunicador social. Obtuvo un máster en Gobierno y Políticas Públicas de la Universidad EAFIT (Colombia) y un máster en Estudios Internacionales de Paz, Conflictos y Desarrollo de la Universitat Jaume I. En la actualidad, realiza estudios de doctorado en Estudios Internacionales de Paz, Conflictos y Desarrollo en la Universitat Jaume I y de Ciencias Sociales, con énfasis en Estudios Latinoamericanos, en la Universidad de Salamanca. Es miembro del grupo de investigación «Mujeres, prácticas culturales y género» de la Universidad de Cartagena (Colombia). Ha participado en importantes conferencias y congresos de Ciencia Política en Inglaterra, Francia, España, México, Ecuador, entre otros.

Marisa García Baldán se inició en la docencia como maestra de educación infantil y primaria en una escuela privada de Barcelona, ciudad en la que nació. Durante más de doce años, también ha ejercido en la Comunidad Valenciana como maestra en la escuela pública. Cursó el máster en Comunicación Intercultural y Enseñanza de Lenguas y el máster en Psicología Positiva Aplicada en la Universitat Jaume I. Actualmente, es profesora asociada en el Departamento de Pedagogía y Didáctica de las Ciencias Sociales, la Lengua y la Literatura de la misma Universidad. Como experta en psicología positiva, su investigación versa sobre el liderazgo y el bienestar en el ámbito educativo. Ha diseñado y está aplicando un programa de intervención en psicología positiva para aumentar el bienestar de los estudiantes universitarios, concretamente en los estudiantes del grado de maestros y maestras de educación primaria, los futuros profesionales de la educación, principales arquitectos de escuelas positivas. 
Gaetano Giannotta (Palermo, 1991) es graduado en Patrimonio Cultural por la Università degli Studi di Palermo (Italia, 2016). Obtuvo el máster en Historia del Arte por la misma universidad en 2018, con la calificación de Sobresaliente Cum Laude. Desde octubre de 2018 realiza estudios de doctorado en Historia del Arte por la Universitat Jaume I de Castellón de la Plana y la Universitat de València, donde está desarrollando un proyecto de tesis doctoral sobre la decoración en estuco en la Valencia del siglo XVIII y sus vínculos técnicos y estilísticos con Italia. Desde abril de 2019 es miembro del proyecto de restauración de la iglesia de los Santos Juanes de Valencia, financiado por la Fundación Hortensia Herrero y coordinado por la profesora Pilar Roig Picazo. Sus publicaciones y participaciones en congresos versan sobre la independencia de la decoración, sus vínculos iconográficos, su conservación y su valorización.

Carlos Hernando Domingo es diplomado universitario en Enfermería por la Universidad de Valencia, licenciado en Educación Física en el INEF de Barcelona y doctor en Educación por la Universitat Jaume I. Es profesor titular de Universidad en el Departamento de Educación y Didácticas Específicas. Desde 1992 dirige el Servicio de Deportes de la Universitat Jaume I, servicio que en 2004 se convirtió en el primer servicio de deportes español universitario en obtener una certificación ISO9001. Ha dirigido dos tesis doctorales, ha participado como ponente en diferentes reuniones científicas y ha publicado diversos artículos en el ámbito del rendimiento en pruebas de larga duración, del deporte universitario y de la didáctica de la Educación Física. Ha participado como investigador principal en diferentes proyectos (Penyagolosa Trail Saludable PTS, Corre Recupera Repite Siempre Saludable CRS, Costa Blanca Trail Saludable CBTS, entre otros).

Jahel Molina Ulldemolins es estudiante de doctorado del programa de Psicología del Deporte y la Salud de la Universidad Autónoma de Barcelona. Es graduada en Psicología por la Universitat Jaume I (2017), donde también cursó estudios de máster en el programa de Investigación Aplicada en Estudios Feministas, de Género y Ciudadanía. Obtuvo el grado de Máster en Psicología del Deporte y el Ejercicio (2018) por el Colegio Oficial de Psicología de la Comunidad Valenciana. Trabaja como técnica y coordi- 
nadora de proyectos a la Federación de Asociación de Estudiantes FADES y colabora con el grupo de investigación MPAGER de la Universitat Jaume I.

Esther Monzó-Nebot es profesora titular del Departamento de Traducción y Comunicación de la Universitat Jaume I. Entre 2013 y 2015 se dedicó a la formación en investigación en el Departamento de Estudios de la Traducción y la Interpretación de la Universidad de Graz (Austria), como catedrática de Sociología de la Traducción y la Interpretación. Su investigación se centra en cómo la traducción y la interpretación contribuyen a la gestión de la diversidad en las sociedades y organizaciones actuales y en cómo traductoras e intérpretes influyen o pueden influir en los desequilibrios de poder estructural y social. Con enfoques psicosociales, realiza estudios descriptivos sobre cómo los habitus y la agencia de traductoras e intérpretes construyen las culturas de la traducción y cómo la competencia intergrupal puede mejorar el trabajo de traductoras e intérpretes en la comunicación y las relaciones entre culturas y grupos sociales. La doctora Monzó es miembro del Instituto Interuniversitario de Filología Valenciana y directora del máster universitario en Investigación en Traducción e Interpretación.

Joan Alfred Noll Obiol se ha procurado una educación multidisciplinaria con unos grados iniciales en Interpretación Musical (clarinete), Ingeniería Técnica de Telecomunicaciones y Sonología. A pesar de haber comenzado su carrera profesional como programador informático, se enfocó más tarde hacia la educación y obtuvo una plaza como funcionario en la Generalidad de Cataluña, donde imparte clases tanto online como presencialmente. Tras darse cuenta de la relevancia de los lenguajes y la traducción en cualquier ámbito del conocimiento humano, obtuvo un Máster en Escritura Creativa (Universidad Pompeu Fabra) y un Master of Science en Traducción Literaria (Universidad de Edimburgo), con una tesis centrada en la traducción de un musical de Sondheim. Actualmente trabaja en su tesis doctoral sobre traducción enlazada con la música en la Universitat Jaume I, tarea que compagina con su trabajo como profesor.

M. Carmen Pastor Verchili es doctora en psicología por la Universitat Jaume I y profesora titular de psicología básica del Departamento de Psicología Básica, Clínica y Psicobiología de la misma universidad. Es coordinadora del 
grupo de investigación MPAGER de la Universitat Jaume I desde diciembre de 2014. Sus principales líneas de investigación básica son el estudio de los sistemas cerebrales implicados en el procesamiento de estímulos emocionales y en las reacciones psicofisiológicas y conductuales ante estos estímulos en muestras normales, clínicas y subclínicas. Ha trabajado en la adaptación a la población española de instrumentos para el estudio de las emociones en el laboratorio, así como instrumentos diagnósticos en el ámbito de la psicopatía y la regulación emocional. Pertenece a sociedades científicas en el ámbito de la neurociencia cognitiva y afectiva (SEPNECA, SPR, APS), es miembro afiliado a Europa del NIMH-CSEA de la Universidad de Florida y del Instituto Universitario de Estudios Feministas y de género «Purificación Escribano» (Universitat Jaume I).

Irina Charlotte Ranaivosoa cursó una Licenciatura en Derecho en la Universidad de Toulouse, Francia, y una Maestría en Derecho en la Universidad de Birmingham, Reino Unido. Su tesis de maestría se centró en la aplicación del derecho humano a la paz en el derecho internacional. Actualmente cursa estudios de paz y ciencias sociales en el Máster Internacional de Estudios para la Paz, Conflictos y Desarrollo de la Universitat Jaume I, España. Su principal interés de investigación es el estado y la implementación de la cultura de la paz en Francia, tema en el que planea centrar sus estudios de doctorado. Ha trabajado como asistente de proyectos para el Colectivo para la Defensa de las Tierras Malgaches (TANY) y ha desempeñado varios cargos en medios sociales, comunicación y desarrollo de proyectos en proyectos comunitarios centrados en la salud y la educación de la juventud en el Reino Unido, Senegal y Vietnam.

Sonia Reverter Bañón es doctora en Filosofía, Profesora Titular de Filosofía del Departamento de Filosofía y Sociología de la Universitat Jaume I y directora del Instituto de Investigación Feminista (IF) de la Universitat Jaume I. Ha sido profesora invitada en las Universidades de Berkeley (USA), European Peace University (Austria), European Peace University (Ireland), Universidad Autónoma del Estado de México, Massachusets Institute of Technology (USA), University of Limerick (Ireland), Peace and Conflict Research Institute (Dinamarca), International Folk School (Dinamarca), University of San Francisco (USA), Gender Institute de London School of Economics (Reino 
Unido), Centre for Civil Society de London School of Economics (Reino Unido), Universidad Pública de Pelotas (Brasil) y la Universidad Nacional del Comahue (Argentina). Sus líneas de investigación prioritarias son la teoría feminista, la crisis del pensamiento europeo contemporáneo, la sociedad civil, la teoría política feminista, el ciberfeminismo, ciudadanía e identidad y el neurogénero.

Ana Vázquez Fernández estudió Traducción e Interpretación en la Universidad de Salamanca, donde se especializó en interpretación. Tiene un Máster en Investigación en Traducción e Interpretación por la Universitat Jaume I y actualmente está terminando el Máster en Lenguas Extranjeras y Lingüística en la Universität Potsdam. Su experiencia en traducción se ha desarrollado en España, Portugal y actualmente en Alemania. Sus intereses de investigación en los estudios de traducción e interpretación incluyen la perspectiva de género, los enfoques bibliométricos, el postcolonialismo y la interseccionalidad. También estudia la comunicación de la ciencia y las intersecciones entre las ciencias naturales y tecnológicas y las ciencias sociales y humanas. 
Els programes internacionals de recerca veuen amb interès creixent la funció de les ciències humanes i socials per traslladar els beneficis de la inversió en ciència i tecnologia a la societat. El coneixement actual de la natura humana i de com l'ésser humà interacciona amb altres individus dins les societats deixa encara moltes incògnites sense resoldre. La recerca en aquests temes, des de les disciplines humanes i socials, té la clau dels grans reptes. La Facultat de Ciències Humanes i Socials crea, amb aquesta col-lecció, un fòrum de debat on potenciar un treball conjunt i multidisciplinari que s'oriente cap a les recerques emergents. La col-lecció Emergents és el testimoni del gran abast de les ciències humanes i socials en un moment de canvi. 
Les ciències humanes i socials han demostrat al llarg de la història una voluntat comuna per fer front als reptes més propers a les persones, els de conviure i comprendre's. Aquest volum recull recerques emergents que agafen aquesta responsabilitat i es posen al capdavant de les societats multiculturals actuals, amb propostes que revisen el passat amb mirades renovades i d'altres que fan visibles els problemes del futur. Amb metodologies i aproximacions teòriques diverses, totes les contribucions d'aquest volum comparteixen l'ambició de generar nou coneixement que ens prepare per resoldre conjuntament els reptes que ens platenja una quotidianeïtat cada vegada més diversa.

\section{IJ UNAUERSITAT}

